\title{
Film als gefundenes Objekt
}

»Wessen Geschichte?«, fragt Yilmaz Dziewior 2009 die Künstlerinnen und Künstler, die er für eine Ausstellung über die Vergangenheit in der Kunst der Gegenwart versammelt - wessen Geschichte wird in der aktuellen »historiografischen Wende in der Kunst« beleuchtet, und vor allem mit welchen Zielen und Mitteln geschieht dies? Ist die von Dieter Roelstraete im selben Band konstatierte Krise der Historiografie dafür verantwortlich, dass sich immer mehr Kunstschaffende und Filmemacher/innen mit ihren Mitteln daran machen, neue ästhetische Wege zu finden, die - politische, persönliche, gesellschaftliche, mythologische - Vergangenheit zu thematisieren und aufzuarbeiten, Dispositive des Erinnerns wie das Archiv, die Sammlung und das Depot, die Ausstellung, digitale Speichermöglichkeiten, aber auch das Fotobuch für sich zu entdecken? Fakt ist, dass sich an der Wende zum 21. Jahrhundert durch die Etablierung der Blackbox für bewegte Bilder nicht nur die Medien der Ausstellung verändern, sondern dass sich die Künstler/ innen auch verstärkt Fragen der Historiografie, Archäologie und Erzählung zuwenden. Auch in der Theoriebildung ist eine Hinwendung zur Geschichte zu beobachten: Insbesondere in den Medien- und Filmwissenschaften werden der Zusammenhang von Erinnerung und Geschichte ${ }^{1}$ sowie der Film als Medium der Historiografie ${ }^{2}$ zuletzt stärker diskutiert, was auch der Wiederentdeckung von Siegfried Kracauers Schriften zur Geschichte zu verdanken ist. ${ }^{3}$ Selbstverständlich erscheint allen Beobachter/innen, dass »die Integration von Material mit historischem Index [...] in künstlerischen Arbeiten allein noch lange keine historische Dimension eröffnet ${ }^{4}{ }^{4}$ Vielmehr müsse, so Susanne

1 Zum Beispiel Matthias Wittmann, MnemoCine. Die Konstruktion des Gedächtnisses in der Erfahrung des Films, Basel 2014; Ute Holl, Matthias Wittmann (Hg.), Memoryscapes. Filmformen der Erinnerung, Zürich/Berlin 2014.

2 Vgl. u. a. Philip Rosen, Change Mummified. Cinema, Historicity, Theory, Minneapolis 2oo1; Mary Ann Doane, The Emergence of Cinematic Time. Modernity, Contingency, the Archive, Cambridge, MA 2002; Simon Rothöhler, Amateur der Weltgeschichte. Historiographische Praktiken im Kino der Gegenwart, Zürich 2011; André Wendler, Anachronismen. Historiografie und Kino, Paderborn 2014.

3 Vgl. Siegfried Kracauer, Geschichte - Vor den letzten Dingen, hg. v. Ingrid Belke, unter Mitarbeit von Sabine Biebl, Frankfurt am Main 2009; Drehli Robnik, Amália Kerekes, Katalin Teller (Hg.), Film als Loch in der Wand. Kino und Geschichte bei Siegfried Kracauer, Wien 2013.

4 Susanne Leeb, »Flucht nach nicht ganz vorn. Geschichte in der Kunst der Gegenwart«, in: Texte zur Kunst 76, Dezember 2009, S. 28-45, S. 42. 
Leeb, eine »imaginäre Dimension $\aleph^{5}$ von Geschichte adressiert werden, um tatsächlich von einem reflexiven, historischen Denken sprechen zu können.

Künstlerische Werke jeder Epoche reflektieren ihre eigene Geschichtlichkeit. Porträts stellen Personen zum Überdauern dar, Historiengemälde erinnern an wichtige Ereignisse in der Geschichte, und Stillleben verweisen durch Memento-Mori-Darstellungen auf die Vergänglichkeit des irdischen Lebens. Insbesondere seit der Erfindung der Fotografie hat der Bezug zur Vergangenheit im Bild eine neue Dimension erhalten. Nicht mehr nur Fragen der Darstellung von Vergangenem in einer bestimmten künstlerischen Form, sondern die verweisende Natur der Technik selbst, ihre Indexikalität, die einige Fototheoretiker, wie wir gesehen haben, mit einem bestimmten Zeitverhältnis in Verbindung bringen, steht nun im Mittelpunkt. Verweise auf tatsächliche oder vergangene Situationen, die in Bildern ausgedrückt werden, bilden einen wichtigen Rahmen für Themen der Darstellung.

Die Rolle der Zeitlichkeit des Bildes für die Darstellung von Geschichte sowie die Reflexion verschiedener Aspekte von Geschichtlichkeit sollen in den folgenden zwei Kapiteln tiefergehend diskutiert werden. Wie in den vorangehenden Besprechungen wird dabei die These leitend sein, dass künstlerische Werke der Wende vom 20. zum 21. Jahrhundert nicht als Visualisierungen einer bestimmten, möglicherweise postmodern zu nennenden historischen Situation und deren Neubestimmung an der historischen und medialen Wende dienen. ${ }^{6}$ Vielmehr stellen die Werke Artikulationsformen dar, die mithilfe ästhetischer Strategien Fragen an die Medien Film, Video und Fotografie und die damit verbundenen Topoi, deren Schnittmengen mit anderen bildnerischen Ausdrucksformen und ihre Bedeutung in künstlerischen Räumen außerhalb des Kinos richten. Im Sinne einer strikten Phänomenologie kommt der Beschreibung der Arbeiten eine große Bedeutung zu, da sich an ihr die Analyse und die Interpretation entwickeln. Ein Hauptaugenmerk liegt auf der in der Werkbetrachtung erfahrbaren Zeitlichkeit der Arbeiten und der Frage nach der >Gegenwärtigkeit` der Zeit des Films, wie sie beispielsweise Gilles Deleuze für das Bewegungs-Bild beschrieben hat, ${ }^{7}$ sowie der Filmerfahrung.

5 Ebd.

6 Dass Kunstwerke durch ihre Darstellung von Zeit auch der Visualisierung von Geschichte dienlich sein können und über bildliche Mittel verfügen, die über die reine Illustration hinausgehen, soll hier nicht bestritten werden, aber als funktionale Dimension von Kunst nicht im Fokus stehen. Vgl. weiterführend Thomas Lange, »Geschichte visualisieren. William Kentridges Felix in Exile« (1994), in: Gamper, Zeit der Darstellung, S. 137-164.

7 »Diese Grundposition schließt ein, dass das Bewegungs-Bild einzig und allein in der Gegenwart ist. Es scheint geradezu selbstverständlich, dass die Gegenwart die einzige direkte Zeit des kinematografischen Bildes ist.« (Deleuze, Das Zeit-Bild, S. 53.) 
Mit Deleuze soll argumentiert werden, dass in jedem in seiner Bewegung als gegenwärtig erfahrenen Filmbild seine andere, virtuelle Seite bereits enthalten ist. Diese zeigt sich in der Sichtbarmachung historischer Verweise im Bild sowie in der Schaffung von Erinnerungs- und Gedächtnisbildern, die den Film als Schichtung verschiedener zeitlicher Ebenen kennzeichnen. Der Terminus Schichtung ist auch in der von Rosalind Krauss geprägten Formulierung der »Schichtung bestimmter Konventionen ${ }^{8} \mathrm{im}$ von James Coleman verwendeten Medium der Diaprojektion enthalten (vgl. Kapitel Beyond Still/Moving. Fotografie und Film im Kunstdiskurs). Er scheint geeignet, zugleich die prismaartige zeitliche Vielseitigkeit und Vielschichtigkeit des filmischen Bildes zu beschreiben, und verweist zugleich auf die künstlerische Praxis der mit Film, Fotografie und Video arbeitenden postmodernen Künstler/innen, welche die historischen Schichten des Mediums in ihren Bildern freilegen.

Den Ausgangspunkt der Analysen bildet ein Sonderfall der Arbeit mit dem bewegten Bild, nämlich die Verwendung von gefundenem, bereits belichtetem oder andersartig codiertem Filmmaterial, sogenanntem Found Footage. Darin wird in besonderer Art und Weise die Verbindung von Geschichtlichkeit und Gegenwärtigkeit thematisiert, weil die historische Ebene des Filmbildes in einem direkten Verweisverhältnis zu mehreren vergangenen Realitäten steht: zu der Zeit der Entstehung des Filmmaterials, der Zeit der Wiederaufnahme, Veränderung oder Neukontextualisierung durch den Künstler oder die Künstlerin, der Zeit der Betrachtung. Die Arbeit mit Found Footage im Kunstraum siedelt sich in einem Randbereich filmischer und künstlerischer Praktiken an. Sie steht bei Douglas Gordon und James Coleman exemplarisch für die Bedeutung, welche die Erinnerung in Bildern und durch Bilder bei ihnen für die Reflexion des Films und für die Konstitution von Geschichte hat. Gordons Arbeiten beruhen auf der Untersuchung des Gedächtnisses, indem er die individuelle Erinnerungsleistung der Betrachtenden herausfordert (aber auch die psychischen Auswirkungen der Dysfunktion des Gedächtnisses thematisiert), Colemans Arbeiten sind indes mit dem Gedanken an Geschichte (im doppelten Wortsinn der historischen Geschichte und der Erzählung) dadurch verbunden, dass er die Zuschauer/innen in die Entstehung eines Geschichtsbildes einbindet. Als eine dritte Position, welche das Verfahren der motivischen Reihung und Serialisierung verfolgt, wird im Vergleich dazu eine Arbeit der deutschen Filmemacher Matthias Müller und Christoph Girardet einbezogen. Die Analyse des Fotobuchs FLOH von Tacita Dean ermöglicht es schließlich, Fragen des Suchen, Findens und Sammelns

8 Krauss, $A$ Voyage on the North Sea, S. 69. 
anhand von Fotografien, die beim Blättern selbst als bewegt erfahren wird, den Methoden des Found-Footage-Films gegenüberzustellen.

\section{Found-Footage-Film - historische Einführung}

Bei Künstler/innen und Filmemacher/innen, die bereits existierendes, gefundenes Bild- und Tonmaterial verwenden, verknüpft sich in einzigartiger Weise die Arbeit eines archäologisch denkenden mit der eines ästhetisch handelnden Subjekts. Das Interesse am Film und seiner (Vor-)Geschichte expliziert sich dabei nicht allein an der Auseinandersetzung mit der Herkunft der Bilder, sondern am Umgang mit dem Material, seiner Sammlung und Strukturierung sowie der Neuinterpretation und Weiterverwendung.

Die Verwendung von bereits existierendem Filmmaterial ist so alt wie die Praxis des Kinos selbst: Bereits Anfang des 20. Jahrhunderts wurden erste Filme aus Resten zusammengeschnitten, um in der Kombination zeitlich, räumlich und kontextuell unterschiedlicher Szenen einen neuen Sinn entstehen zu lassen. ${ }^{9}$ Die Gattung des Kompilationsfilms entstand aus dem ökonomisch motivierten Bedürfnis, mit vorhandenem Material - aus Schnittabfällen oder aber Bildern mit besonderem Sensationswert - eine neue oder veränderte Geschichte zu erzählen. ${ }^{10}$ Christa Blümlinger weist jedoch zu Recht darauf hin, dass die Wiederverwendung von Vorgefundenem im Film an sich noch keine ästhetische Transformation impliziert, sondern dem Medium durch Montage und Reproduktivität bereits eingeschrieben ist. ${ }^{11} \mathrm{Im}$ Kompilationsfilm, aus dem das Genre der Wochenschau hervorging, geht es um die »Organisation dokumentarischer Erzählungen auf Basis von Archivmaterial $\ll .{ }^{12}$ Er bildet den Ausgangspunkt für den vor allem in den 195oer Jahren mit Bruce Conner Bedeutung erlangenden avantgardistischen Found-FootageFilm, der auf dem Prinzip der Collage basiert und bis heute einen festen Bestandteil des Experimentalfilms darstellt. Diente der Kompilationsfilm noch einer häufig durch Kommentar unterstützten Narration, zeichnen sich die avantgardistischen Experimental- und essayistischen Dokumentarfilme durch das Bestreben aus, sich »gewissermaßen archäologisch zu situieren, mit vorherrschenden Konventionen zu brechen, eine Differenz zu markieren oder

Zur Geschichte der Wiederverwendung von Filmmaterial vgl. Jay Leyda, Filme aus Filmen. Eine Studie über den Kompilationsfilm, Berlin 1967; William Charles Wees, Recycled Images. The Art and Politics of Found Footage Films, New York 1993; Christa Blümlinger, Kino aus zweiter Hand. Zur Ästhetik materieller Aneignung im Film und in der Medienkunst, Berlin 2009 .

10 Leyda, Filme aus Filmen, S. $11 \mathrm{f}$.

11 Blümlinger, Kino aus zweiter Hand, S. 9.

12 Ebd. 
Neues zu gestalten.$^{13}$ Sie arbeiten dabei nicht nur nahezu ausschließlich mit gefundenem Material, sondern machen dies auch zum Thema. ${ }^{14}$ Die Variation reicht dabei von der unkommentierten Aneignung gefundener Filmrollen (Ken Jacobs, Perfect Film, 1986) über die metaphorische, oft ironische oder subversive Bedeutung, welche durch Kollisionsmontage thematisch unterschiedlicher Einstellungen erzeugt wird (Bruce Conner, A Movie, 1958), und die Porträtierung von und Hommage an einzelne Filmstars (Joseph Cornell, Rose Hobart, 1936) bis zur zurückhaltenden Analyse historischen Materials durch Dehnung oder Vergrößerung (Ken Jacobs, Tom Tom the Pipers Son, 1969, Erni Gehr, Eureka, 1974) und zur Freilegung versteckter Bedeutungsschichten im Hollywoodfilm durch die isolierte Wiederholung einzelner, zum Beispiel gestischer Momente (Martin Arnold, Pièce Touchée, 1993). Die Arbeit mit Found Footage, so wird deutlich, bezeichnet kein Genre, sondern eine Haltung zum filmischen Material, die Auswirkungen auf das Verständnis der Rolle des Künstlers oder der Künstlerin als Autor/in sowie auf den Werkbegriff hat.

Im Experimentalfilm ist eine konstante Präsenz des Found-Footage-Films seit den 196oer Jahren zu beobachten. Verschiedene Richtungen des strukturellen Films in Europa und den USA setzten dabei unterschiedliche Akzente, entweder auf die Stofflichkeit und Bearbeitung des Materials ${ }^{15}$ oder auf die formalästhetischen Spezifika des Films. In den späten 1970er und 198oer Jahren traten vor allem in der Wiener Filmszene Künstler/innen hervor, die sich in der Nachfolge von Peter Kubelka und Kurt Kren mit dem Erbe der Avantgarde auseinandersetzten und mit der Entwicklung der Videokunst und der Videotechnik konfrontiert sahen (hier sind insbesondere Dietmar Brehm, Lisl Ponger, Peter Tscherkassky und Martin Arnold zu erwähnen). Die logistischen, technischen und ästhetischen Möglichkeiten der Videotechnik unterstützten ab den 198oer Jahren die Migration des Found Footages in Dispositive jenseits des Kinos, brachten aber auch eine neue Generation

13 Ebd.

14 Aufgrund der Vielgestaltigkeit der Möglichkeiten, Found Footage als Teil eines künstlerischen Verfahrens einzusetzen, sind alle Autor/innen um Ab- beziehungsweise Eingrenzung des Gegenstands bemüht. William Wees trifft einerseits graduelle Unterscheidungen im Hinblick auf die Quantität des gefundenen Materials und die Selbstreflexivität der Filme (Wees, Recycled Images, S. 4), andererseits unterscheidet er zwischen Kompilation, Collage und Appropriation als historisch bewusste, kritische und unkritische/illustrative Art der Aneignung vorgefundenen Filmmaterials. Christa Blümlinger fragt insbesondere nach der Art und dem Grad der erzeugten Historizität (Blümlinger, Kino aus zweiter Hand, S. 10 f., S. 32).

15 Beispielsweise Birgit und Wilhelm Hein mit Rohfilm (1968) oder George Landow aka Owen Land mit Film in Which There Appear Sprocket Holes, Edge Lettering, Dirt Particles, Etc. (1965/66). 
Found-Footage-Künstler/innen hervor, deren Material- und Archivbegriff sich grundlegend änderte. Filmemacher wie Jean-Luc Godard, Chris Marker und Harun Farocki kombinierten die klassische Filmmontage in »filmischen und videographischen Palimpsesten $\aleph^{16}$ mit Überblendungen, Doppelprojektionen und Bildstillstellungen, wobei sie die veränderte Haptik und Taktilität der Filmarbeit als größten Unterschied betonen:

Nicht nur die Art des Bildes - einmal als fotografisch entwickeltes Einzelbild, einmal als abstrakte, bildlose Information auf dem Magnetband - setzen [sic] den Unterschied zwischen Film und Video, sondern auch die daraus resultierende Operationalität. ${ }^{17}$

Die Arbeit am Schneidetisch, bei der das fotografisch belichtete Zelluloidmaterial per Hand und Optical Printer Bild für Bild bearbeitet und wieder zu neuen Filmen zusammengefügt wird, gewinnt angesichts der produktionsund präsentationstechnischen Veränderungen, mit denen sich Filmemacher/innen zu Beginn des 21. Jahrhunderts konfrontiert sehen, auch für ihr ästhetisches Selbstverständnis an Bedeutung - dies betont zum Beispiel Peter Tscherkassky. ${ }^{18}$ Nicht umsonst führt Christa Blümlinger am Schluss ihrer Studie noch einmal die verschiedenen Formen des Übertritts des Films in den Kunstraum auf, die von dialogischen Kunst-Kino-Ausstellungen über Filminstallationen bis hin zu einem Plädoyer für ebenjenen handgemachten Found-Footage-Film als »metafilmische«, materialbewusste Archivkunst reichen. ${ }^{19}$

Nur erwähnt werden in ihrer aus filmwissenschaftlicher Sicht verfassten Studie die Werke, deren Stoßrichtung nicht primär materialästhetischer oder filmhistorischer, sondern konzeptueller Natur ist, wozu sie unter anderem Douglas Gordons und Pierre Huyghes Found-Footage-Videoarbeiten zählt. ${ }^{20}$ Und tatsächlich unterscheidet sich der Zugang der jüngeren Videokünstler/ innen, die vor allem seit den 199oer Jahren mit filmischen Fundstücken

16 Blümlinger, Kino aus zweiter Hand, S. 269.

17 Volker Pantenburg, Film als Theorie. Bildforschung bei Harun Farocki und Jean-Luc Godard, Bielefeld 2006, S. 178.

18 »Aber ich arbeite mit dem Material, und der Begriff des Physischen spielt eine sehr große Rolle bei mir: ich will eine physische Reaktion auslösen mit dem Kino und seiner Apparatur, das dieser Dinosaurier da oben, der Projektor, vor 1oo Jahren möglich gemacht hat.« (Volker Pantenburg, »Ein physisches Kino. Gespräch mit Peter Tscherkassky«, 7.7.2008, Österreichisches Filmmuseum, Wien, http://www.kunst-der-vermittlung.de/ dossiers/filmvermittelnde-experimentalfilme/gespraech-tscherkassky/ [26.4.2020].)

19 Blümlinger, Kino aus zweiter Hand, S. 271.

20 Ebd., S. 32. 
international in Ausstellungen vertreten sind, ${ }^{21}$ von den klassischen FoundFootage-Filmen vor allem dadurch, dass sie die Korrespondenzen zwischen Bildern nicht nur mittels innerfilmischer Montage und Überblendungstechniken, sondern auch in der Ausstellungssituation beispielsweise durch die Kombination von freistehenden Leinwänden erzeugen. Sie reflektieren dabei, wie im Weiteren ausgeführt werden soll, nicht nur die historische und symbolische Bedeutung der verwendeten Filme, sondern auch ihre Sichtbarkeit im Rahmen von fernsehbasierter $>$ Archivarbeit $\measuredangle$, die sich in der spezifischen Videoästhetik, ihrer oberflächlichen Durchlässigkeit und in charakteristischen Artefakten wiederspiegelt. Wenn Blümlinger das Prädikat »metafilmisch « ausschließlich dem material- und dispositivbewussten, im engen Sinn selbstreflexiven Found-Footage-Film zuspricht, soll dem entgegengehalten werden, dass gerade die nicht im engen Sinn filmischen Aneignungsstrategien den Film als Medium und Erzählform reflektieren und, wie bei James Coleman gezeigt werden wird, die affektive und somatische Dimension des Leinwandgeschehens durch die Aktualisierung im Zuschauerkörper herausstellen.

Eine Unterscheidung kann hier auf der Ebene der Materialität und Stofflichkeit, nicht jedoch hinsichtlich der Historizität getroffen werden. Blümlinger argumentiert, Gordons Arbeiten zielten nicht auf das »Nachleben der Bilder oder eine andere Dimension von Historizität, sondern auf die unmittelbare Gedächtnisleistung des Zuschauers $a b \ll{ }^{22}$ Wie gezeigt werden wird, greift diese Kritik jedoch zu kurz, da sie nicht berücksichtigt, dass auch Gordons konzeptuelle Werke Ausdruck eines historischen Interesses sind; dieses widmet sich jedoch weniger der >Erzeugung`von Historizität als vielmehr der Frage nach den Bedingungen ihrer Zuschreibung.

\section{Film als somatisches Spektakel}

James Colemans Box (ahareturnabout) von 1977 ist eine von zwei Arbeiten innerhalb eines sich über vier Jahrzehnte erstreckenden Werks, in denen er

21 Hier sind exemplarisch zu erwähnen: Stefano Basilico (Hg.), Cut. Film as a Found Object in Contemporary Video, Ausstellungskatalog Milwaukee Art Museum 2004, Milwaukee 2004; Joachim Jäger, Gabriele Knapstein, Anette Hüsch (Hg.), Jenseits des Kinos. Die Kunst der Projektion. Filme, Videos und Installationen von 1963 bis 2005, Ausstellungskatalog Hamburger Bahnhof, Berlin 2006/2007, Ostfildern 2006; Kerry Brougher (Hg.), The Cinema Effect. Illusion, Reality, and the Moving Image, Ausstellungskatalog Hirshhorn Museum and Sculpture Garden, Smithsonian Institution, Washington, DC, 2008, London 2008.

22 Blümlinger, Kino aus zweiter Hand, S. 32. 
Found Footage verwendet. Diese und die Diainstallation La Tache Aveugle von 1978/9o nehmen eine besondere Stellung in seinem Werk ein, entstanden sie doch am Ende einer Periode, in welcher Coleman sich mit wahrnehmungstheoretischen Fragen beschäftigte und bereits mit der Rhythmisierung des visuellen Eindrucks experimentierte, die in Box (ahareturnabout), aber auch in seinen späteren Diainstallationen eine Rolle spielt. Die zeitliche Strukturierung der Wahrnehmung und die darin wichtige Funktion der Erinnerung untersuchte er beispielsweise in Flash Piece, einer Installation, die zuletzt Ende der 198oer Jahre zu sehen war und aus blauen und gelben Blitzen besteht, welche abwechselnd in wiederholten minütlichen Zyklen erscheinen. Anne Rorimer beschreibt:

Während jedes Zyklus war der zeitliche Abstand zwischen den Blitzen unterschiedlich, trotzdem erinnerten die Betrachter sich daran, als ob sie alle gleich gewesen wären. Die gemessene Zeit und der persönliche Zeiteindruck stimmten nicht überein. Dementsprechend gelang es Coleman, einen neuen subjektiven Aspekt des Sehens - nämlich den der Erinnerung - in das Subjekt der resultierenden Arbeit einzuführen. ${ }^{23}$

In diesen frühen Arbeiten widmet sich Coleman der Zeiterfahrung und damit verbunden der Funktionsweise der subjektiven Erinnerung. ${ }^{24}$ Die beiden Werke, in denen er Archivmaterial beziehungsweise Einzelbilder aus einem Spielfilm verwendet, kündigen eine Veränderung in seinem Werk an, das in den folgenden Jahren von einer starken Auseinandersetzung mit der Repräsentation von Wirklichkeit in den Medien Fotografie, Film, Literatur und Theater geprägt sein wird.

\section{Beschreibung Box (ahareturnabout) (1977)}

Um Colemans Arbeit Box zu sehen, betritt man einen dunklen Raum, in dem bereits vor der Sichtbarkeit des Films ein lauter, rhythmisch erklingender Ton hör- und spürbar wird. Es erscheint ein Schwarz-Weiß-Film, wandfüllend, auf einer Seite des Raumes, der - in der bei Sportberichterstattungen üblichen Vogelschauperspektive - einen Ausschnitt des Vierecks eines Boxrings und zwei sich darin befindende Boxer zeigt. Am Rande des Boxrings ist das Publikum zu erkennen. Die Betrachter von Colemans Arbeit ssehen< hier jedoch keinen Film im herkömmlichen Sinne, sondern nur sehr kurze Abschnitte von drei bis zehn Bildern, also Sekundenbruchteile, die sich mit etwas längeren Schwarzblenden abwechseln. Die kurzen Filmausschnitte

23 Beschreibung von Anne Rorimer, zit. n. Buchloh, »James Coleman«, S. 101.

24 Siehe hierzu auch die Arbeiten Slide Piece (1973) und Clara and Dario (1974). 
werden von dem sehr lauten, alles durchdringenden Puls begleitet, der aus im Raum aufgestellten Lautsprechern schallt, so dass der Ton den Raum ganz ausfüllt und das Vibrieren der Lautsprecher spürbar ist. Doch auch die Schwarzblenden sind nicht stumm: Neben dem gleichmäßigen Rattern des ebenfalls im Raum aufgestellten Filmprojektors ist ein Voiceover einer männlichen Stimme zu hören, die Worte äußert, laut und rasselnd atmet und keucht. Die Worte stehen zum Teil in Zusammenhang mit dem gesehenen Boxkampf, so kann man Aufforderungen vernehmen wie »again, again«, »push «, »box «, »hold it « sowie Worte, die Körperteile (»the liver«) oder Elemente der Kampfsituation benennen (»the ref«, »the bout«). Sie beziehen sich zum Teil direkt auf vorher ausgeführte Handlungen wie einen Schlag oder eine Umklammerung. Darüber hinaus können auch nicht direkt auf den Kampf bezogene Worte erkannt werden, die einen über das Ereignis hinausgehenden, möglicherweise ebenfalls gewalttätigen und darüber hinaus politischen Bedeutungszusammenhang andeuten (»the sticks «, »the wood «, »not capital's «). ${ }^{25}$ Die Zuordnung der Stimme ist zunächst nicht deutlich - sie kann ebenso zu einem mitfiebernden Kommentator des Kampfes gehören oder aber die innere Stimme eines der Boxer repräsentieren. Einem Interview mit James Coleman ist jedoch zu entnehmen, dass es sich um einen inneren Monolog von Gene Tunney, dem irischen Sieger des Kampfes, handeln soll. ${ }^{26}$ Der Gesamteindruck ist ein in variierenden Abschnitten aufblitzendes, kontrastreiches Bewegtbild, das mit einem lauten Puls die Schwärze durchbricht, die von einer gehetzten, emotionalen und aggressiven Stimme erfüllt ist. Ein Durchlauf der im Loop gezeigten Installation dauert 4 Minuten 50 Sekunden (Abb. 9).

Die das Publikum umgebende Dunkelheit der Blackbox wird als Schwärze empfunden, ist jedoch nur theoretisch als solche wahrnehmbar; praktisch produziert der visuelle Eindruck auf der Netzhaut der Betrachter/innen ein optisches Nachbild, das in der Dunkelheit anstelle des belichteten Materials vor den Augen aufblitzt. Der Effekt der Interaktion belichteter und schwarzer Kader verweist auf ein Phänomen, das aus dem Experimentalfilm als Flicker bekannt ist. Hierbei wird derWechsel von weißem und dunklem (oder farbigem) Licht ab einer bestimmten Frequenzbreite als getrennte Impulse registriert. ${ }^{27}$ Zwar beruht jede Filmprojektion auf der Unterbrechung eines belichteten Streifens, dabei wird das Licht jedoch aufgrund der hohen Frequenz ab

25 Vgl. auch das Transkript des Voiceovers in Jean Fisher, »James Coleman«, in: James Coleman, Ausstellungskatalog The Douglas Hyde Gallery, Dublin, The Arts Council of Northern lreland Gallery, Belfast, Dublin 1982, S. 5-26, S. 17.

26 Richard Kearney, »Interview with James Coleman«, in: The Crane Bag 6/2, 1982, S. 127-132, S. 128 .

27 Vgl. Hein, Herzogenrath, Film als Film, S. 33 . 


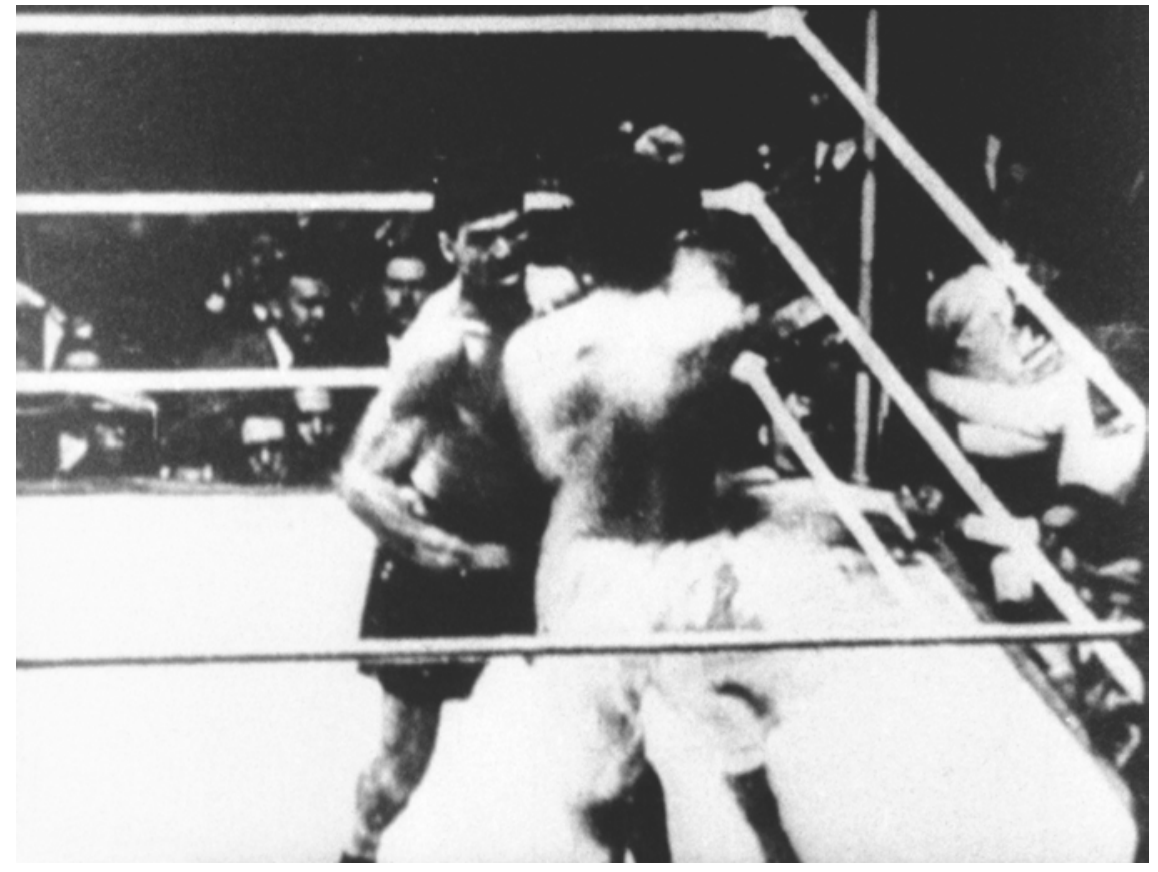

Abb. 9 James Coleman, Box (ahareturnabout), 1977 (@ James Coleman) 
ca. 5 o Hertz als kontinuierliche Lichtquelle wahrgenommen. Beim Flicker, der in den 196oer Jahren erstmals im Film verwendet wurde, können Nachbilder auf der Netzhaut entstehen, welche sich mit dem neuen Lichtimpuls vermischen und so Bilder erzeugen, die nur in der optischen Wahrnehmung existieren. ${ }^{28}$ Wird dieses Phänomen zur Gestaltung von Filmen eingesetzt, werden zumeist in schnellem Wechsel schwarze und weiße Kader arrangiert, ähnlich einem Stroboskoplicht, jedoch nicht gleichförmig, sondern rhythmisch, mit sanfteren und schnelleren Flickerpassagen sowie überwiegend `schwarzen< beziehungsweise >weißen< Passagen. ${ }^{29}$ Das Ziel dabei ist, im Sinne der Freilegung der filmischen Struktur durch Reduzierung der Mittel den Film von jedem repräsentativen Gegenstandsbezug zu befreien und in seiner reinen Materialität zu zeigen. Der Flicker ist nach P. Adams Sitney eines der Hauptelemente des strukturellen Films, jener kurzen, stark selbstreflexiven Phase der Filmavantgarde, in der die Grundelemente des filmischen Sehens und des filmischen Apparats in experimentellen Wahrnehmungsstudien untersucht wurden:

The structural film insists on its shape, and what content it has is minimal and subsidiary to the outline. Four characteristics of the structural film are its fixed camera position (fixed frame from the viewer's perspective), the flicker effect, loop printing, and rephotography off the screen. Very seldom will one find all four characteristics in a single film, and there are structural films which modify these usual elements. ${ }^{30}$

Rosalind Krauss bemerkt zum Flickerfilm, dass er durch die Entstehung der Nachbilder sein eigentliches Ziel, nämlich durch den Eingriff in die zeitliche Struktur des Films hinter die Bewegungsillusion zu blicken und in dem Einzelbild das basale Element des Films in seiner Materialität sehen zu können, verfehlt habe:

28 Ebd.

29 Flicker ist nahezu zeitgleich in Europa und den USA erstmals Grundlage eines Films verwendet worden (Peter Kubelka, Arnulf Rainer, 1958-196o, 35 mm, 6:30 min., schwarzweiß, Ton; Tony Conrad, The Flicker, 1966, 35 mm, 30 min., schwarz-weiß, Ton). Die Filme unterscheiden sich insbesondere hinsichtlich ihres Erkenntnisinteresses, wie Birgit Hein beschreibt: »Während es Kubelka in >Arnulf Rainer um eine visuelle Komposition nach musikalischen Prinzipien geht, ist Conrads >The Flicker< das Ergebnis systematischer Untersuchung über den stroboskopischen Effekt in der Wahrnehmung.«(Hein, Herzogenrath, Film als Film, S. 183.)

30 P. Adams Sitney, Visionary Film. The American Avant-Garde 1943-2000, 3. Auflage, New York 2002, S. 348. 
The flicker film was invented to stop time, to disable the afterimage's perceptual mechanism by means of which the visual >persistence $<$ of information contained in one film frame would bleed into the next, creating the illusion of an uninterrupted flow of movement. ${ }^{31}$

Das Nachbild werde im Flicker so erst recht zum Gegenstand der Wahrnehmung: »[T] he afterimage [...] now has a place to exist within which it can be experienced as the ghostly counterpart to the passages of filmic representation. ${ }^{32}$ Es bewirke, dass wir in den Zwischenräumen nicht die materielle Oberfläche des Films, sondern die Auswirkungen des neuronalen Feedbacks unseres eigenen Wahrnehmungsapparats sähen. Krauss' Annahme jedoch, dass das positive Nachbild die Grundlage der Sichtbarkeit von Bildbewegung ist, geht von einem physiologischen Irrtum aus, der spätestens seit den 1970er Jahren durch wahrnehmungsphysiologische Untersuchungen widerlegt wurde. ${ }^{33}$ Tatsächlich haben Nachbildeffekt (persistence of vision) ${ }^{34}$ und die Wahrnehmung einer Bildbewegung wenig miteinander $\mathrm{zu}$ tun, sondern Bewegung wird im projizierten Bild auf der Grundlage des Stroboskopeffekts gesehen - die Nachbildwirkung beziehungsweise der Phi-Effekt >verbessern< die so gesehene Bewegung durch die Überblendung der Nachbilder auf der Netzhaut.

\section{Phänomenologische Ebene der Filmbetrachtung}

Im Weiteren bietet es sich an, die Positionen der Filmtheorie und -ästhetik mit einzubeziehen, die sich explizit mit der körperlichen Affizierung durch den Film beschäftigt haben. Dies ist von Bedeutung für die Beschreibung der Zeitlichkeit und Geschichtlichkeit in Colemans Arbeit, weil sich, wie weiter unten argumentiert wird, die Vergegenwärtigung des historischen Geschehens nur mittels der leiblichen Erfahrung im Ausstellungsraum einstellt. Den

31 Rosalind E. Krauss, »Pulse«, in: Yve-Alain Bois, dies., Formless. A User's Guide, New York 1999, S. 161-165, S. 161.

32 Ebd.

33 Siehe hierzu Joseph und Barbara Anderson, »Motion Perception in Motion Pictures«, in: Lauretis, Heath, The Cinematic Apparatus, S. 76-95, sowie Bill Nichols, Susan L. Lederman, »Flicker and Motion in Film«, in: Lauretis, Heath, The Cinematic Apparatus, S. 96-105. In den letzten Jahren wurde von einigen Autor/innen die Frage nach der filmischen Bewegung wieder aufgenommen und dementsprechend nicht als Effekt eines visuellen Defekts im Auge, sondern als Figuration auf der Leinwand untersucht. Beispielhaft hierzu seien erwähnt: Gunning, »Moving Away from the Index«, und Joachim Paech, »Der Bewegung einer Linie folgen ... Notizen zum Bewegungsbild«, in: ders., Der Bewegung einer Linie folgen. Schriften zum Film, Berlin 2002, S. 133-161.

34 Siehe zum Phänomen und zum Terminus >persistence of vision< in der Filmliteratur Anderson, »Motion Perception in Motion Pictures«, S. 96 f. 
Zusammenhang von leiblicher Wahrnehmung und Filmerfahrung hat insbesondere Vivian Sobchack in The Address of the Eye ${ }^{35}$ zur Grundlage ihrer Theorie gemacht, in der sie das Sehen eines Films mit der Phänomenologie der Wahrnehmung von Maurice Merleau-Ponty in Verbindung bringt. Im Gegensatz zu den damals vorherrschenden psychoanalytischen, kognitivistischen und sprachwissenschaftlichen Theorieansätzen in der Filmwissenschaft geht Sobchack von dem Filmzuschauer als wahrnehmendem Subjekt aus, dessen somatische, sinnliche Reaktion auf das filmische Medium die dem intellektuellen und kognitiven Verständnis vorgängige Grundlage aller Filmerfahrung bildet. Sobchack charakterisiert die Filmerfahrung als kommunikatives System, das auf der körperlichen Wahrnehmung als Vehikel der bewussten Erfahrung beruht; Sinn wird nicht nur visuell, sondern auch auditiv und haptisch erzeugt. Film versteht sie nicht als Repräsentation und Reflexion einer vom Regisseur vorgeplanten Erfahrung, sondern als Präsentation der direkten Wahrnehmungserfahrung. ${ }^{36}$ Das Erleben des Films ist dabei trotzdem ein reflektierter Akt, der das Leinwandgeschehen als körperliche Erfahrung zu reflektieren vermag:

Watching a film is both a direct and mediated experience of direct experience as mediation. We both perceive a world within the immediate experience of an >other< and without it, as immediate experience mediated by an >other<. Watching a film, we can see the seeing as well as the seen, hear the hearing as well as the heard, and feel the movement as well as see the moved. ${ }^{37}$

Wenn so der Zuschauerkörper zum »Leihkörper« des Films wird, wie Christiane Voss es ausdrückt, reduziert sich die in der projizierten Zweidimensionalität begründete Abstraktion des Films: Der erfahrende Körper wird zur dritten Dimension und zum konstitutiven Bestandteil der filmischen Architektur. ${ }^{38}$ Leinwandgeschehen und Filmerfahrung stehen dabei nicht nur dialektisch, sondern auch dialogisch zueinander: »The film's vision and my own do not conflate, but meet in the sharing of a world and constitute an experience that is not only intrasubjectively dialectical, but also intersubjectively dialogical.« ${ }^{39}$

Sobchacks Ansatz erhielt in den 199oer Jahren viel Aufmerksamkeit, da er es ermöglichte, vom okularzentristischen Paradigma des Kinos, wie es

35 Vivian Carol Sobchack, The Address of the Eye. A Phenomenology of Film Experience, Princeton, NJ 1992.

36 Ebd., S. 9.

37 Ebd., S. 10.

38 Christiane Voss, »Filmerfahrung und Illusionsbildung. Der Zuschauer als Leihkörper des Kinos«, in: Koch, dies., ... kraft der Illusion, S. 71-86, S. 81.

39 Sobchack, The Address of The Eye, S. 24. 
beispielsweise die psychoanalytische Filmtheorie verfolgte, abzurücken. ${ }^{40}$ Obgleich Sobchack nur über die Filmerfahrung im Kino spricht und die hier verhandelten Werke sich von dieser schon allein aufgrund der institutionellen und räumlichen Situation unterscheiden, kann der Grundgedanke der phänomenologischen Dimension der Filmerfahrung auch auf bewegte Bilder im Ausstellungsraum übertragen werden und möglicherweise gerade hier besonders greifen, da bei diesen Werken der ästhetischen Erfahrung häufig ein dem ästhetischen Objekt ebenbürtiger, wenn nicht höherer Stellenwert zugesprochen wird. ${ }^{41}$

Bei James Colemans Box (ahareturnabout) wird das Publikum auf mehreren Ebenen in das Werk einbezogen. Die nach Sobchack erste und unvermeidbare Ebene ist die der körperlichen Erfahrung der bewegten Bilder, welche hier affektiv und multisensual ist. Sie wird durch die überwältigenden Effekte der Lautstärke - welche als spürbare Schwingung nicht nur über das Ohr, sondern auch über die Haut körperlich aufgenommen wird - und des Flickers in der Dunkelheit des Ausstellungs- beziehungsweise Aufführungsraums so gesteigert, dass ein distanziertes Umhergehen und Betrachten der Bilder nicht möglich ist. Der zugleich optische und auditive pulsierende Reiz verhindert eine rein visuelle Erfahrung, ${ }^{42}$ da zum einen der Raum von der Geräuschkulisse und auch dem Rattern des Filmprojektors ausgefüllt ist, zum anderen der optische Reiz ein retinales Echo erzeugt. Dem lauten Beat kann sich die Wahrnehmung nicht verschließen, wie Dorothea von Hantelmann ihre Erfahrung beschreibt:

[I]m Moment des Betretens erreichte er die Grenze des Erträglichen, fast gewaltsam ergriff der Rhythmus vom Körper des Betrachters Besitz und ließ ihn zum Resonanzkörper eines Kunstwerks werden, noch bevor man es richtig erfasst hat. [...] Das visuelle Stakkato mit den starken Schwarzweißkontrasten traf das Auge nicht weniger gewaltsam als der akustische Beat den Rest des Körpers. ${ }^{43}$

40 Gleichwohl muss der Kritik von Malin Wahlberg Rechnung getragen werden, dass auch Sobchack - wenn auch in weit geringerem Maße als andere feministische Filmtheoretikerinnen wie Laura Mulvey oder Kaja Silverman - im Anschluss an MerleauPonty einen Schwerpunkt auf den Blick legt. Dies wird auch im obigen Zitat deutlich, wo in Bezug auf die im Weiteren als audiovisuell und taktil beschriebene Filmerfahrung wiederholt von »watching « gesprochen wird. Vgl. Malin Wahlberg, Documentary Time. Film and Phenomenology, Minneapolis 2008, S. $18 \mathrm{f}$.

41 Vgl. Elsaesser, Hagener, Filmtheorie zur Einführung, S. 153.

42 Dies führt Rosalind E. Krauss aus in ihrem Essay »The Im/pulse to See«, in: Foster (Hg.), Vision and Visuality, S. 51-75.

43 Hantelmann, How to do things with art, S. 34. 
Die körperliche Erfahrung der Situation geht, wie von Hantelmanns Beschreibung illustriert, jedem kognitiven Verständnis des Werks voraus. Die aufblitzenden Bildsequenzen sind so kurz, dass es zwar möglich wird, einen Boxkampf zu erkennen, jedoch nicht, seinem Verlauf zu folgen. Verfolgungen durch den Ring wechseln sich mit gegenseitigem Taxieren und kurzen Schlagabtäuschen ab, deren visuelle Wucht mit dem akustischen Puls koinzidiert, so dass beide Reize sich gegenseitig verstärken und ein Miterleben des Leinwandgeschehens forciert wird. ${ }^{44}$ Auch ohne zu wissen, um welchen Kampf es sich hier handelt - die Bildqualität lässt darauf schließen, dass es altes Filmmaterial ist -, ist der Betrachter oder die Betrachterin von der Brisanz der dargestellten Szene überzeugt, was nicht zuletzt durch den angestrengten Kommentar unterstützt wird, dessen Intonation den dramatischen Verlauf des Kampfes nachfühlen lässt.

An dieser Stelle muss nach dem Stellenwert des historischen Materials gefragt werden: Ausgehend von der Annahme, dass Coleman ein Geschichtsbild entwirft, das im Zusammenwirken der gegenwärtigen Werkerfahrung mit der historischen Dimension des Werks entsteht, kann die Arbeit nicht auf die Gegenwärtigkeit ihrer Aufführung reduziert werden, sondern es muss der Semantik des Materials Rechnung getragen werden oder, wie Buchloh es formuliert:

[I]n eben dem Maße wie Box die Erfahrung des perzeptuellen Pulses im Betrachter wortwörtlich und psychophysiologisch einschreibt [...] wird in gleichem Maße - und als Paradox - die ikonische Dimension der Abbildung und die textuelle Dimension des inneren Monologs diesem Puls als dialektisches Gegenprinzip gegenübergestellt. ${ }^{45}$

Bei den Kämpfern im Film handele es sich so auch nicht um »transhistorische Figuren «, sondern um »historisch spezifische Subjekte «, ${ }^{46}$ nämlich um die Protagonisten in einem Kampf von historischer Tragweite, der

44 Man könnte den Beat in Box (ahareturnabout) in seiner doppelten Funktion - den Betrachter oder die Betrachterin affizierend und in die Werkgenese einbindend, gleichzeitig jedoch auch die Bildbewegung unterbrechend - möglicherweise mit dem gleichsetzen, was Rosalind Krauss als traumartige zeitgleiche Erfahrung des Äußeren und des Inneren eines Zootrops beschreibt: »the beat both constructing the gestalt and undoing it at the same time - both positioning us within the scene as its active viewer and outside it as its passive witness. (Krauss, »The Im/pulse to See«, S. 59). Der Puls oder Beat unterbricht die Wahrnehmung der Bildbewegung und fragmentiert sie.

45 Buchloh, »James Coleman«, S. 89.

46 Ebd., S. 90. 
am 22. September 1927 in Chicago zwischen dem Iren Gene Tunney und dem Amerikaner irischer Abstammung Jack Dempsey stattfand. Er ist legendär, weil Tunney, der dem seit 1919 amtierenden Champion Dempsey im Jahr zuvor den Schwergewichts-Weltmeistertitel abgenommen hatte, diesen hier verteidigen musste. Er gewann den Kampf knapp und beendete seine Karriere ein Jahr später ungeschlagen. Der Sieg ist jedoch bis heute umstritten, da Tunney möglicherweise nur aufgrund eines in der Boxgeschichte außergewöhnlichen Ereignisses, dem sogenannten long count, gewann, einer Unregelmäßigkeit im Anzählen. ${ }^{47}$ Tunney zählte als junger, strategischer und technikbasierter Kämpfer, der mit Dempsey den langjährigen Champion mit dem härtesten Schlag herausgefordert hatte und überraschenderweise den Titel errang. Die historische Bedeutung des Kampfes in der Geschichte des Boxens ist unbestritten, da Tunney seinen Titel verteidigte, Dempsey sich vom Profiboxen zurückzog, und der long count diese Entwicklung somit womöglich einleitete.

Der Grund für die Auswahl gerade dieses Filmmaterials für die Arbeit muss jedoch in der Bedeutung des Ereignisses für das historische Subjekt gesucht werden. Diese erhält es, weil Gene Tunney »sowohl seine Rollenidentität als Boxchampion wie auch seine ethnische Identität als Ire in diesem Kampf zu retten hatte « ${ }^{48}$ James Coleman interessierte vor allem der unklare Zustand, in dem sich der Titelverteidiger im Moment des Kampfes befand: Als Verteidiger der eigenen Identität als Champion ist er in einer zeitlichen Schleife gefangen, er ist >nicht mehr $<$ und `noch nicht $<$, und somit auf seine eigene Körperlichkeit als irreduziblen Rest zurückgeworfen.

Unter Berücksichtigung der Tatsache, dass Box (ahareturnabout) in den 1970er Jahren auf dem Höhepunkt des Nordirlandkonflikts entstand, kann die Darstellung des irischen Außenseiters in einer gewalttätigen Auseinandersetzung noch auf eine weitere historische Ebene, den Freiheitskampf Nordirlands, verweisen. Dass mit Box (ahareturnabout) nicht ausschließlich der historische Boxkampf gemeint ist, sondern auch die blutige irische Geschichte in ihrem Kampf um Unabhängigkeit eine Rolle spielt, klingt in den irischen Wörtern und Kampfelementen an, die im Voiceover zu entziffern sind, ${ }^{49}$ ist aber auch in der Thematisierung der irischen Frage in späteren Werken Colemans impliziert.

47 Tunney ging in der siebten Runde zu Boden, eine neue Regel besagte jedoch, dass erst angezählt wurde, wenn der andere Boxer sich in einer neutralen Ecke befand. Dempsey befolgte die Anweisung des Schiedsrichters nicht sofort, so dass Tunney sich einige Sekunden länger erholen konnte. Diese Sekunden könnten ihm, so wird unter Experten und Sporthistorikern spekuliert, zum Sieg verholfen haben.

48 Buchloh, »James Coleman«, S. 91.

49 Hantelmann, How to do things with art, S. 35. 
Die Verweise in Box (ahareturnabout) jedoch bleiben stets unkonkret, die Arbeit erzielt ihre Wirkung in erster Linie auf der Ebene der dargestellten und erlebten Körperlichkeit, impliziert dabei aber die latente, nicht artikulierte historische und kulturelle Tragweite als gegeben und unhintergehbar. Den Konflikt, den Coleman hier auch mit der Tradition des Postminimalismus austrägt, formuliert Benjamin Buchloh wie folgt:

Seine Praxis konstituiert sich gerade in der emphatischen Rettung der Dimension des Gedächtnisses wie in der präzisen, annihilierenden Kritik der gegenwärtigen Bedingungen visueller Erfahrung. ${ }^{50}$

Gerade in diesem historischen Detail von Box (ahareturnabout) kulminiere Colemans kontinuierliche Bezugnahme auf die historische Dimension der ästhetischen Darstellung.

Dass die Erinnerung an kulturell und politisch bedeutsame Ereignisse auch über die Medien gesteuert und über Bilder allererst ermöglicht wird, mit denen subjektive Erinnerungen narrativiert und Geschichten und Mythen geformt werden, darauf verweist Coleman selbst in einem Interview. ${ }^{51}$ Die filmische Aufzeichnung des Kampfes diente im Nachhinein der Dokumentation von Tunneys Sieg, aber auch als Beleg, dass das Anzählen ungewöhnlich lange gedauert hatte, und bietet die Grundlage für bis heute andauernde Spekulationen über die Rechtmäßigkeit des Sieges. Tunney wurde zum tragischen Helden, dessen Triumph immer unsicher bleiben wird und den Coleman in seiner Arbeit gegen das seinem Sieg stets als Möglichkeit anhaftende Scheitern ankämpfen lässt. Das historische Ereignis offenbart sich in Box (ahareturnabout) als immer wieder neu zu verhandelnder Möglichkeitsraum, es wird zum aktualisierten, neuen $>$ Sich-Ereignen< des Kampfes. ${ }^{52}$

Benjamin Buchloh stellt zudem die Bedeutung des Boxens im Sinne einer Archäologie des Spektakels heraus:

Es ist in dieser Hinsicht auch keineswegs unbedeutend, dass Coleman gerade den locus classicus der Spektakelkultur zum Gegenstand seiner Arbeit macht: der öffentliche Kampf zweier (athletischer) Rivalen hat Künstler im zwanzigsten

50 Buchloh, »James Coleman«, S. 91.

51 »Richard Kearney: [...] The Tunney-Dempsey film projection exposes the ways in which the media of western culture make collective heroes out of tortured split-personalities (for example: the sound track of Tunney's interior monologue). James Coleman: Very true. In fact a good title might have been Hit and Myth!« (Kearney, »Interview with James Coleman«, S. 128).

52 Mit Derrida könnte man das Ereignis in der ständigen Repetition der Arbeit - Repetition des Kampfes und des Pulses - als dekonstruiert beschreiben. 
Jahrhundert nicht nur als eine Urform des Spektakels, sondern immer wieder als eine fundamentale Metapher allgemeiner sozialer Beziehungen fasziniert. ${ }^{53}$

Es ist jedoch keine allgemeine, sondern eine sehr konkrete, männliche Beziehung, die hier zur Schau gestellt wird. Die männlichen, weißen Subjekte, deren körperlicher Machtkampf im Boxen, dem maskulinen Rollenritual par excellence, ausgetragen wird, werden zum Gegenstand eines medialen Großereignisses und bieten sich in Colemans Arbeit auch noch 50 Jahre später für den körperlichen Nachvollzug des für den amtierenden Champion existenziellen Kampfes durch die Betrachter/innen an. Der gesprochene innere Monolog setzt dabei auf einer textuellen Ebene der vermeintlichen Evidenz des fotografischen Bildes eine subjektiv-imaginative Dimension entgegen.

Das historische Ereignis des Kampfes wird mit dem gegenwärtigen Ereignis der Werkkonstitution auf Basis der körperlichen Einbeziehung des Betrachters / der Betrachterin verknüpft. Diese Verwobenheit ist so stark, dass sich das Verhältnis von repräsentiertem Kampf und somatischer Resonanz auszutauschen scheinen: Die Beteiligung des eigenen Körpers an der Werkkonstitution bewirkt, so Krauss, »that Box's subject-matter is somehow displaced away from the representational place of the sporting event and into the rhythmic field of two sets of beats or pulses: the viewer's and the boxer's «.54 Mark B. Hansen interpretiert dies als eine Konvertierung des rhythmischen Boxens im Filmmaterial in den Rhythmus der körperlichen Reaktion der Zuschauer/innen. ${ }^{55}$

Das Werk kann, so wird deutlich, nur in der direkten Rezeption im Ausstellungsraum angemessen erfahren werden - dies trifft auf alle Arbeiten Colemans zu, die sich durch einen besonderen Aufführungscharakter auszeichnen. Der Künstler nimmt sich als Autor dabei ernst: Jedes Detail der Arbeit unterliegt seiner absoluten Kontrolle, bis hin zur Dämmung der Wände des Raumes. Dennoch ist die Werkstruktur offen, denn das Werk aktualisiert sich in jedem Moment neu in der Erfahrung durch die teilnehmenden Zuschauer/innen. Bei Box (ahareturnabout) findet sich die von Dorothea von Hantelmann in ihrer Analyse der Arbeit akzentuierte Performativität als Ereignishaftigkeit auf allen drei Ebenen: der Werkstruktur, der Thematik und der Wirkungs- beziehungsweise Erfahrungsdimension: Die Arbeit hängt von ihrer Aufführung ab, sie thematisiert ein theatrales, ritualisiertes Spektakel und ist nur als ein auf die Betrachtenden hin geöffnetes Werk zu denken. Der

53 Buchloh, »James Coleman«, S. 9o - so auch zitiert von Hantelmann, How to do things with art, S. 56 .

54 Krauss, »Pulse «, S. 163 .

55 Hansen, New Philosophy for New Media, S. 28. 
Zusammenklang des Rhythmus, des historischen Bildes, der sinneren< Stimme und der optischen Nachbilder ergibt sich nur in der Erfahrung. ${ }^{56}$ Das ausgewählte Filmmaterial wird daher nicht als Spur oder Repräsentation eines vergangenen Ereignisses vorgeführt, sondern in der ästhetischen Erfahrung des rhythmischen Erlebens vergegenwärtigt.

\section{Aufblitzen der Vergangenheit: Box (ahareturnabout) als dialektisches Bild}

Diese FigurderVergegenwärtigungeines Gewesenen erinnertan die Konzeption des sogenannten dialektischen Bildes bei Walter Benjamin, das er in seinen >Thesen zur Geschichte` als eine Figur beschreibt, die das Erinnern mit der Geschichte zu verknüpfen vermag. ${ }^{57}$ Das dialektische Bild soll im Vergleich mit Gilles Deleuzes Zeit-Bild hier herangezogen werden, um zu zeigen, dass das blitzartige Aufscheinen der Vergangenheit im Film eine Entsprechung in der theoretischen Bestimmung des Erinnerns als Mittel der Geschichtserfahrung hat. Benjamins Geschichtsphilosophie geht unter anderem von einer Kritik am Historismus als Akkumulation historischer Fakten auf der Grundlage von Fortschrittsdenken aus; er setzt diesem narrativen und kontinuierlichen Konzept von Geschichte die Idee einer dialektischen Geschichtskonzeption entgegen. In dieser erhellen sich Gegenwart und Vergangenheit sprunghaft gegenseitig, ohne jedoch in einer Synthese im Hegel'schen Sinne aufzugehen. Vielmehr wird so die Vergangenheit als Bild in der Gegenwart instantan sichtbar. Es ist dabei wie das Filmbild flüchtig: »Das wahre Bild der Vergangenheit huscht vorbei. Nur als Bild, das auf Nimmerwiedersehen im Augenblick seiner Erkennbarkeit eben aufblitzt, ist die Vergangenheit festzuhalten.« ${ }^{58}$ Die Aktualisierung des Gewesenen im »Jetzt der Erkennbarkeit« ist die dialektische Figur, welche Erkenntnis als Bild möglich macht:

Nicht so ist es, daß das Vergangene sein Licht auf das Gegenwärtige oder das Gegenwärtige sein Licht auf das Vergangne wirft, sondern Bild ist dasjenige,

56 Die gegenseitige Abhängigkeit zwischen dem, was das Werk >sagt<, also zeigt, und was es stut<, als Wirkung hervorbringt, sei dabei das - nach Austin - performative Element der Arbeit.

57 Vgl. Walter Benjamin, »Über den Begriff der Geschichte«, in: ders., Erzählen. Schriften zur Theorie der Narration und zur literarischen Prosa, ausgewählt und mit einem Nachwort von Alexander Honold, Frankfurt am Main 2007, S. 129-140. Auch Dorothea von Hantelmann bezieht sich in ihrer Analyse von Box (ahareturnabout) auf den Geschichtsbegriff Walter Benjamins, siehe Hantelmann, How to do things with art, S. 42-47. Sie verknüpft ihre Analyse jedoch, wie bereits in Kapitel 3 erläutert wurde, mit der Performativitätstheorie Austins.

$5^{8}$ Benjamin, »Über den Begriff der Geschichte«, S. 131. 
worin das Gewesene mit dem Jetzt blitzhaft zu einer Konstellation zusammentritt. Mit andern Worten: Bild ist die Dialektik im Stillstand. Denn während die Beziehung der Gegenwart zur Vergangenheit eine rein zeitliche ist, ist die des Gewesnen zum Jetzt eine dialektische: nicht zeitlicher sondern bildlicher Natur. ${ }^{59}$

Gemeint ist hier ein mentales Erinnerungsbild, denn der Erinnerung kommt in Benjamins Geschichtsphilosophie eine konstitutive Rolle zu: »[Sie] wiederholt nicht die Ereignisse der Vergangenheit, sondern gestaltet sie in neuer Form. $\ll 0$ Die Zeitlichkeit des dialektischen Bildes steht einer prozesshaft gedachten, fortschrittsorientierten Zeit diametral entgegen und konterkariert so auch jede lineare, teleologische Geschichtsvorstellung. Zeit erscheint hier konsequenterweise »im Stillstand «, da der eigentlich in der Zeit ablaufende dialektische Prozess im Bild verdichtet wird. Bezogen auf Box (ahareturnabout) kann von einer bildhaften Entsprechung dieser Konzeption gesprochen werden: In dem Werk stellt sich ein historisches Ereignis dar, jedoch nicht über die Einbettung in eine Geschichte oder durch die Präsentation von Fakten, sondern im buchstäblichen Aufblitzen des Vergangenen in der gegenwärtigen Erfahrung. Diese filmische Erfahrung ist »schockartig ${ },{ }^{61}$ diskontinuierlich, sie verdichtet sich, wie oben beschrieben, in der unmittelbaren Wahrnehmung des Betrachters oder der Betrachterin zu einem kontrastreichen Bewegtbild, in dem das Vergangene in Fragmenten - in in sich bewegten Einzelbildern - herausgestellt, stillgestellt wird. Wie in Colemans Diainstallationen, die in erzählender, jedoch nichtsdestotrotz diskontinuierlicher Form das Standbild in seinem Wesen erschüttern und eigentlich, wie Kaja Silverman an INITIALS beschreibt, aus einem einzigen, lange pulsierenden Foto bestehen, ${ }^{62}$ zeigt sich in $B o x$ (ahareturnabout) der Kampf als ein einziges langes, pulsierendes Bewegtbild. Es ist jedoch nicht nur die somatische Vergegenwärtigung des Bildes als Puls, welche die Vergangenheit zum dialektischen Bild verdichtet, sondern auch die Tonspur. Indem Coleman das historische Bildmaterial mit einem vermeintlichen inneren Monolog von Tunney koppelt, welcher aus der Aufführung

59 Walter Benjamin, Das Passagen-Werk, Bd. 1, hg. v. Rolf Tiedemann, Frankfurt am Main 1983 , S. 578.

6o Detlev Schöttker, »Erinnern«, in: Michael Opitz (Hg.), Benjamins Begriffe, Bd. 1, Frankfurt am Main 200o, S. 26o-298, S. 265.

61 Auf die »Chockwirkung « des Films hat Benjamin bekanntlich in seinem Kunstwerkaufsatz hingewiesen, vgl. Walter Benjamin, »Das Kunstwerk im Zeitalter seiner technischen Reproduzierbarkeit« (1935), in: ders., Das Kunstwerk im Zeitalter seiner technischen Reproduzierbarkeit. Drei Studien zur Kunstsoziologie, Frankfurt am Main 2003, S. 7-44, S. 39 .

62 Kaja Silverman, »Im Wachsen begriffen«, in: Friedel (Hg.), James Coleman, S. 50-71, S. 69. 
heraus den Blick in das Ereignis zurückwendet, jedoch dabei stets im Hier und Jetzt der auditiven Wahrnehmung verbleibt, fügt er zwischen die zeitliche Ebene des Filmmaterials und die Zeit der ästhetischen Erfahrung der Arbeit eine dritte Zeitebene ein, welche die Entstehung des Found Footages als neues Werk mittels der Interpretation des Materials durch den Künstler umfasst.

Das Erinnern ist bei Benjamin stets gegenwartsbezogen (er verwendet den Begriff des Erwachens); die »Idee des historischen Erinnerns als Vergegenwärtigung der Vergangenheit « ${ }^{63}$ dient bei ihm im Kontext seines marxistischen Geschichtsverständnisses dem Zweck der Gegenwartserkenntnis. Der Gedanke, dass Gegenwart und Vergangenheit in einem Bild aufeinander verwiesen sind, findet sich in ähnlicher Weise, wenn auch von einer anderen philosophischen Position her gedacht, bei Gilles Deleuze, der diese Verdichtung direkt auf das Filmbild bezogen hat. ${ }^{64}$

\section{Gegenwartsspitzen und Vergangenheitsschichten}

Einen Versuch, sich aus filmphilosophischer Sicht dem Verhältnis von Wahrnehmung, Vergangenheit und Geschichte zu nähern, bietet die Konzeption des Zeit-Bildes von Gilles Deleuze in seinem zweiten Kinobuch, wo er die Ablösung des aktionsgebundenen Bewegungsbildes durch das Zeit-Bild, welches das moderne Kino kennzeichnet, beschreibt. Die Zeitlichkeit des Bildes bekommt hier, wie bereits im ersten Kapitel erläutert, eine neue Wendung, indem ihr neue Charakteristika zugeschrieben werden: Im Gegensatz zum BewegungsBild, dessen Zeit unabdingbar an die Bewegung gekoppelt ist - und wo die Bewegung als Aktion oder Ereignisfolge im Bild immer >sensomotorisch $<$ ist -, ist hier nun erstmals die Zeit nicht der Bewegung untergeordnet, sondern die Bewegung der Zeit:»Diese Umkehrung macht nicht mehr aus der Zeit das Maß der Bewegung, sondern aus der Bewegung eine Perspektive der Zeit. « ${ }^{65}$ Diese >falschen Bewegungen< des Zeitbildes können sich auf der inhaltlichen und auf der formalen Ebene, in Bildgestaltung und Bildanschluss zeigen. ${ }^{66}$ Das Verhältnis von Vergangenheit und Gegenwart beziehungsweise der Status von

63 Schöttker, »Erinnern«, S. 281.

64 Der Vergleich der Erinnerungs- und Geschichtskonzeptionen von Benjamin und Deleuze findet sich in ähnlicher Weise, wie er hier vorgenommen wird, auch bei Olaf Berg (»Benjamin und Deleuze. Ansätze für eine kritische Geschichtswissenschaft in Filmbildern«, in: Zeitschrift für kritische Theorie 22-23, 2006, S. 68-98). Berg betrachtet diese Konzeptionen jedoch nicht unter dem Blickwinkel künstlerischer Aneignung, sondern begreift Film als historische Forschung. Er verfolgt das Ziel, den Film für die Geschichtswissenschaft fruchtbar zu machen.

65 Deleuze, Das Zeit-Bild, S. 37.

66 Vgl. Schaub, Gilles Deleuze im Kino, S. 175. 
Gedächtnis und Erinnerung im Zeit-Bild wird am deutlichsten im Kristallbild, dem Bildtypus, in dem in einer unhintergehbaren Doppelheit das aktuelle mit (s)einem virtuellen Bild zu einem zweiseitigen oder gespaltenen Bild verknüpft ist: $67 »$ Und in der Tat gibt es kein Virtuelles, das nicht durch Bezug auf das Aktuelle aktuell würde, während dieses innerhalb derselben Beziehung virtuell wird: wir haben es hier mit vollständig umkehrbaren Vorder- und Rückseiten zu tun. $\ll^{68}$ Das Kristallbild kann in Motiven wie dem Spiegel aufscheinen, sich aber auch auf die dem Film zugrunde liegende Struktur beziehen. Das Aktuelle und das Virtuelle stehen dabei in stetiger Wechselwirkung und ständigem Austausch.

Deleuze bezieht sich hier wie bereits in Das Bewegungs-Bild auf Henri Bergson, der die Frage nach dem aktuellen und dem virtuellen Bild zeitlich verstanden hat. In Materie und Gedächtnis (1986) ${ }^{69}$ beschreibt Bergson den Zusammenhang von Wahrnehmung und Erinnerung als Wechselwirkung der Zeitebenen, als ein gegenseitiges Durchdringen von Gegenwart und Vergangenheit. Für Bergson ist das Aktuelle an die Gegenwart gekoppelt, doch diese Gegenwart macht bereits im Augenblick ihres Vergehens einer neuen Gegenwart Platz und wird zur Vergangenheit. Das Vergangene - bezogen auf das Gedächtnis in der Form der »reinen Erinnerung « - hört dabei nicht auf zu bestehen, sondern wird »nutzlos«, kontemplativ, träumerisch, solange es nicht in der Gegenwart zum Bild aktualisiert wird:

Von meiner Vergangenheit wird nur das zum Bilde und folglich zur Empfindung [...], was bei dieser Tätigkeit mitarbeiten kann, sich der Haltung einfügen, mit einem Wort sich nützlich machen kann; aber sobald meine Vergangenheit Bild wird, verlässt sie den Zustand der reinen Erinnerung und schmilzt mit einem Teil meiner Gegenwart zusammen. ${ }^{70}$

Deleuze schlussfolgert daraus, dass das Erinnerungsbild gegenwärtig und vergangen zugleich sei: »Die Gegenwart ist das aktuelle Bild, und seine zeitgleiche Vergangenheit ist das virtuelle Bild, das Spiegelbild. « ${ }^{71}$ Vergangenheit und Gegenwart seien koexistent, insofern die Zeit sich »in jedem Augenblick in [...] vorübergehende Gegenwart und sich bewahrende Vergangenheit « ${ }^{72}$ aufteile.

\footnotetext{
67 Vgl. Oliver Fahle, »Zeitspaltungen. Gedächtnis und Erinnerung bei Gilles Deleuze«, in: montage $A V$ 11/1, 2002, S. 97-112.

68 Deleuze, Das Zeit-Bild, S. 97.

69 Henri Bergson, Materie und Gedächtnis: eine Abhandlung über die Beziehung zwischen Körper und Geist, Hamburg 1991 (1896).

70 Ebd., S. 135 .

71 Deleuze, Das Zeit-Bild, S. 108 f.

72 Ebd., S. 113 .
} 
Die von Bergson für den Prozess der Erinnerung beschriebene Formung des (Erinnerungs-)Bildes in der Wahrnehmung findet Deleuze im modernen Film wieder. ${ }^{73}$ Er schließt an die Bergson'sche Bestimmung der Zeit an, wenn es ihm um die Rolle der Erinnerung geht, die er im Zeit-Bild in zweifacher Art sichtbar werden sieht. Die Vergangenheit erscheint dabei »als die allgemeinste Form eines Schon-da [déjà-là], [...] die unsere Erinnerungen voraussetzen « ${ }^{74}$ Die Gegenwart existiert folglich »selbst nur als eine unendlich zusammengezogene Vergangenheit, die sich auf der äußersten Spitze des Schon-da konstitutiert «. ${ }^{75}$ Deleuze beschreibt zwei Arten von Zeitvorstellungen (Chronozeichen) als voneinander getrennte Modi: die Konzeption der Zeit von der Gegenwart her, das heißt die Gesamtheit der Zeit als Gegenwart, die in Spitzen oder Akzenten auftritt (»Simultaneität der Spitzen der de-aktualisierten Gegenwart« im Affekt oder Ereignis bei Robbe-Grillet), und die Gesamtheit der Zeit als »Architektur« von Vergangenheitsschichten (»Koexistenz der Schichten der virtuellen Vergangenheit« bei Resnais).${ }^{76}$ Es handelt sich um zwei voneinander getrennt existierende Pole, die miteinander verbunden sind. ${ }^{77}$ Diese Verbindung der beiden Pole zu einem Zeit-Bild wird Deleuze zufolge in Phänomenen wie der Schärfentiefe bei Orson Welles sichtbar.

Für die Betrachtung von Box (ahareturnabout) aus film- und geschichtsphilosophischer Sicht interessiert jedoch der grundsätzliche Gedanke der gleichzeitig getrennt existierenden Zeitsphären, die sich als aktuelle und virtuelle Seite des Bildes - hier: des Werks - in einem kristallinen Zustand unablässig erneuern. Das aktuelle Bild ist nicht ohne seine - virtuelle - Vergangenheit zu denken, das Bild ist, wie beschrieben, gespalten und verschmolzen zugleich (wie bei Benjamin ist hier nicht von einer vereinenden Synthesis der korrelierenden Zeitebenen die Rede). Es können also in der Deleuze'schen Konzeption des Zeitbildes »Vergangenheitsschichten« mit

$73 \gg$ Das Gedächtnis ist ein Ort der Organisation von Bildern der Vergangenheit [...], und der Film, so Deleuze, ist das Medium zur ästhetischen Erforschung dieses Bereichs.«(Fahle, »Zeitspaltungen «, S. 101.)

74 Deleuze, Das Zeit-Bild, S. 132.

75 Ebd., S. 133. Diese »paradoxen Eigenschaften einer achronologischen Zeit« - PPäexistenz einer Vergangenheit im allgemeinen, die Koexistenz aller Vergangenheitsschichten, die Existenz einer am meisten zusammengezogenen Stufe - sind Voraussetzung für die dialektische Beziehung, die Vergangenheit und Gegenwart im kinematografischen Erinnerungsbild zum Beispiel in Citizen Kane, eingehen. Deleuze bezieht sich hier auf das Kegelschema in Bergsons Materie und Gedächtnis, 3. Kapitel.

$7^{6}$ Deleuze, Das Zeit-Bild, S. 140.

77 Ebd., S. 146. 
»Gegenwartsspitzen « in Bildern verknüpft sein, in denen die Vergangenheit als Virtualität - als Erinnerungsbild - Teil eines aktuellen Bildes ist. ${ }^{78}$

Man kann Colemans Box (ahareturnabout) und darüber hinaus möglicherweise alle Arbeiten, die ihr geschichtliches Material in einer neuen Form aufgehen lassen, als ein solches Zeit-Bild beschreiben. Das Ausgangsmaterial ist dabei wohlgemerkt ein reines Bewegungsbild: Es bildet einen zeitlichen Verlauf ab, den Kampf, dessen Bewegungen einer sensomotorischen Logik innerhalb eines räumlichen und zeitlichen Kontinuums folgen. Erst in seiner Fragmentierung und in seiner Koexistenz im Bild, im Ton und im Betrachter wird das sensomotorische Band >zerrissen<, erscheint ein Bild, dessen Bewegungen seiner zersplitterten Zeit unterworfen sind.

Deleuze setzt dabei einen etwas anderen Akzent als Benjamin, beide Konzepte von Bild und Erinnerung tragen jedoch gleichermaßen zur Erhellung der Arbeit bei. Während Benjamins dialektisches Bild aus einem gegenwärtigen Erkenntnisinteresse heraus ein Bild der Vergangenheit aufblitzen lässt, um in dem schockartigen Stillstand die Kohärenz beider Zeiten herzustellen (hier: des historische Boxkampfs und der Gewalttätigkeit der ästhetischen Erfahrung, die den irischen Kampf ums Überleben vor Augen führt), ähnelt das Deleuze'sche Erinnerungsbild einem archäologischen Freilegen von Vergangenheitsschichten im gegenwärtigen Bild, in dem das aktuelle und das virtuelle Bild koexistieren. Walter Benjamin benutzt ebenfalls die Metapher des Kristalls in Bezug auf die Montage als Prinzip der marxistischen Geschichtsschreibung:

[D]ie erste Etappe dieses Weges wird sein, das Prinzip der Montage in die Geschichte zu übernehmen. Also die großen Konstruktionen aus kleinsten, scharf schneidend konfektionierten Baugliedern zu errichten. Ja in der Analyse

78 Die Verknüpfung herzustellen gesteht er dem rezeptiven Prozess beim Lesen eines Buches, beim Betrachten eines Schauspiels oder eines Bildes zu; die zeitliche Schnur legt dann der Rezipienten / der Rezipientin (oder der Autor / die Autorin) als eine Art »Transformationsschicht, die eine Art transversaler Kontinuität oder Kommunikation zwischen verschiedenen Schichten erfindet und zwischen ihnen eine Gesamtheit nichtlokalisierbarer Relationen webt« (vgl. ebd., S. 164). Dies sei jedoch ein fragiler, schwieriger Prozess. Präzedenzfall für ein Kunstwerk, dem dies von selbst gelingt, ist für Deleuze Alain Resnais' L'année dernière à Marienbad: »Es kann jedoch vorkommen, dass es dem Kunstwerk gelingt, jene paradoxen, hypnotischen und halluzinatorischen Schichten zu erfinden, deren Charakteristikum darin besteht, gleichzeitig eine Vergangenheit, aber immer auch eine zukünftige Vergangenheit zu sein. [...] Das Kunstwerk durchquert die koexistierenden Epochen, außer wenn es daran gehindert ist, wenn es, in einer mortifizierten Fragmentierung, auf eine erschöpfte Schicht fixiert ist (Les Statues meurent aussi).«(Ebd., S. 164 f.) 
des kleinen Einzelmoments den Kristall des Totalgeschehens zu entdecken. Also mit dem historischen Vulgärnaturalismus zu brechen. Die Konstruktion der Geschichte als solche zu erfassen. In Kommentarstruktur. ${ }^{79}$

Es scheint kein Zufall zu sein, dass von Benjamin und Deleuze mit dem Blitz und dem Kristall - der ja wie ein Prisma wirken kann - jeweils optische Metaphern gewählt werden, um den Zusammenhang von Bild, Zeit und Geschichte zu beschreiben.

In dem dialektischen Zeit-Bild, das Box (ahareturnabout) produziert, erscheint keine >reine Erinnerung<; das historische Material gibt sich als solches zu erkennen, stellt aber in seiner projizierten Flüchtigkeit nur einen prekären Bezug zum vergangenen Ereignis her. Durch die Abhängigkeit der Arbeit von der sensorischen Wahrnehmung der Betrachter/innen wird das historische Material nicht >mortifiziert «, man betrachtet keine Rückblende oder Ähnliches, sondern es stellt einen direkten Bezug zur körperlichen, zerebralen, emotionalen und politischen Lebenswirklichkeit der Betrachtenden her.

James Colemans Box (ahareturnabout) ist in seiner physischen Präsenz und starken Beteiligung der Betrachter/innen an der Wirkung des Werks sicherlich ein Sonderfall innerhalb der Arbeit mit Found Footage in filmischen Installationen. Es ist trotzdem aussagekräftig, weil sich in ihm die Zeitlichkeit nicht über die Dauer des Bildes mitteilt wie in einigen der vorhergehenden Werke, sondern über die absolute Gegenwärtigkeit im Akt der Wahrnehmung. Dass sich gerade während der Aufführung in der leiblich erfahrenen Gegenwart des Found-Footage-Materials die Erkenntnis von Geschichte einstellt, unterscheidet Colemans Werk von historiografischen Ansätzen, die eine sprachliche und kognitive Erfassung des historischen Ereignisses anstreben. Parallelen jedoch finden sich zum Beispiel in der amerikanischen Filmavantgarde wie bei Bruce Conner oder Paul Sharits. Das Werk von Sharits hat sich in ähnlicher Weise den Fragen nach optischen Phänomenen, der zeitlichen Strukturierung der Wahrnehmung und der Ästhetik traditioneller Bildprinzipien gewidmet. Sharits' und Conners Found-Footage-Filme haben thematische Strukturen, sie kompilieren und bearbeiten Bilder, denen politische, psychologische oder sexuelle Bedeutung zugewiesen wird, sie sehen ihre Filme aber nicht als Geschichten. ${ }^{80}$ Paul Sharits' Film Epileptic Seizure Comparison (1976) ist mit Box (aharaeturnabout) vergleichbar, weil fotografisches Filmmaterial mit der

79 Benjamin, Das Passagen-Werk, Bd. 1, S. 575.

8o Vgl. Paul Sharits, »Allgemeine Erklärung für das 4. Internationale Experimentalfilmfestival in Knokke-le-Zoute, Belgien, 1967«, zit. n. Susanne Pfeffer (Hg.), Paul Sharits. Eine Retrospektive, Begleitheft zur Ausstellung im Museum Fridericianum, Kassel, 23.11.201422.2.2015, Kassel 2014, nicht paginiert. 
somatischen Wirkung eines Flickereffekts kombiniert wird. In einer Doppelprojektion übereinander werden zwei Filme mit Footage von epileptischen Anfällen stationärer Patienten gezeigt. Diese Bilder sind stroboskopartig mit farbigen Kadern durchsetzt, die einen Flickereffekt erzeugen, der durch die Auskleidung der konisch zulaufenden Ausstellungsarchitektur mit Metallfolie noch verstärkt wird: »Wenn die Zuschauer den Raum betreten, werden sie ganz vom pulsierenden Licht umschlossen. Der Stereoton schirmt dabei wie eine Mauer den Raum nach außen ab. So entsteht das Gefühl, selbst an dem Anfall, wie ihn die Bilder zeigen, teilzuhaben. ${ }^{81}$ Eine Tonspur, die auf der Frequenz der Gehirnwellen während eines Anfalls basiert, begleitet die Filme. Sharits selbst bescheibt: »EPILEPTIC SEIZURE COMPARISON ist der Versuch, Ton- und Lichtrhythmen in einem entsprechend proportionierten Raum so zu organisieren, daß eine Situation entsteht, in der auch Nicht-Epileptiker unter kontrollierten Bedingungen die Gewalt eines Krampfanfalles nachempfinden können. ${ }^{82}$ Sein Ziel ist somit nicht, über die körperliche Affizierung hinaus die historische Bedeutung des Materials offenzulegen, sondern den Betrachter oder die Betrachterin in das sich im Moment ereignende, zerebrale Gewitter des Anfalls hineinzuversetzen. Ebenso, wie ein epileptischer Anfall keine zeitliche Dimension außer der Gegenwart kennt, kennt auch dieses Werk keine solche.

\section{Fazit}

In der Analyse von Box (ahareturnabout) vor dem Hintergrund phänomenologischer, film- und geschichtsphilosophischer Ansätze wurde gezeigt, dass die Arbeit ihre Wirkung im Spannungsfeld verschiedener zeitlicher und räumlicher Ebenen entfaltet. Grundlage für das Zusammenwirken dieser Ebenen ist die leibliche Betrachterwahrnehmung, welche die Freilegung beziehungsweise Aktualisierung historischer Schichten im Werk erst ermöglicht. Zwar lassen sich die historischen Bezüge auch unabhängig von der körperlichen Wahrnehmung benennen, die Realisierung als gewalttätige Erfahrung, die mit der irischen Identität des Boxers (und des Künstlers) untrennbar verknüpft zu sein scheint, kann jedoch unabhängig von der Leiblichkeit der Betrachtenden nicht gedacht werden. Die Verbindung dieser Voraussetzung mit den Gedanken zur Bildwerdung von Erinnerung und Geschichte bei Benjamin und Deleuze scheint zunächst ungewöhnlich, schreibt Vivian Sobchack doch selbst, dass ihre Phänomenologie der Filmwahrnehmung nur sehr wenig mit der Kinotheorie von Deleuze gemein habe, auch weil dieser eine fundamentale

81 Beschreibung der Arbeit in: Kinemathek 25/72 (Paul Sharits), Juli 1988, S. 29.

82 Ebd. 
Unterscheidung zwischen der »natürlichen Wahrnehmung « bei MerleauPonty und der Filmwahrnehmung mache. ${ }^{83}$ Auch betont Sobchack, dass ihre Studie stärker die räumliche denn die zeitliche Disposition in den Fokus rücke: »It is not time, but space - the significant space lived as and through the objective body-subject, the historical space of situation - that grounds the response to those questions and the question of cinematic signification in this present study. «84 Dennoch kann, wie die obigen Ausführungen zeigen sollten, eine Kombination der verschiedenen Ansätze von Sobchack, Deleuze und Benjamin für die Arbeit fruchtbar gemacht werden, da im »cinästhetischen Körper «, 85 der die starre Subjekt-Objekt-Trennung von Leinwandgeschehen und Zuschauerposition aufhebt und beides umgreift, gerade durch die Resonanz des Körpers die absolute Gegenwärtigkeit der blitzhaften Erfahrung mit dem Erkennen der historischen, narrativen und medialen Sedimente der Arbeit einhergeht: »Die starke, an den Moment der Wahrnehmung gebundene Erfahrung, die Colemans Arbeit so emphatisch erfahrbar macht, ist zugleich auf Geschichtliches bezogen, oder besser: sie trägt Geschichtliches in sich. ${ }^{86}$

Box (ahareturnabout) ist dabei insofern eine geschichtliche Arbeit und erstellt ein Geschichtsbild, als dieses von vornherein als >Prisma< mit verschiedenen Ansichten und Brechungsgraden konzipiert ist - das heißt, je nach Blickwinkel offenbart sich eine andere Dimension von Geschichtlichkeit. Die materielle Beschaffenheit und Bildästhetik des Films verraten seinen analogen und >alten< Ursprung; darüber hinaus kann er im Sinne einer Medienarchäologie auf die filmischen Darstellungskonventionen des Sportspektakels, im Sinne einer geschlechterspezifischen Analyse auf die Determinierung des männlichen Körpers durch diese Konventionen oder im Sinne der nationalen Identität der Boxer auf die blutige Geschichte des irischen Unabhängigkeitskampfs hin untersucht werden. Christa Blümlinger stellt als wesentliche Eigenschaft des »historisch denkenden« Found-Footage-Films die Differenz zum Ausgangsmaterial heraus, ${ }^{87}$ die hier gegeben ist, auch wenn es sich nicht um einen neu montierten Film aus verschiedenen Versatzstücken handelt. Als Elemente der >Collage< können vielmehr die drei Ebenen des bewegten Bildes, der Sprache und des Pulses bezeichnet werden, in deren Zwischenräumen sich die Erfahrung von Historizität ereignet.

83 Sobchack, The Address of The Eye, S. 31: »Deleuze, however, neglects the embodied situation of the spectator and of the film.«

84 Ebd.

85 Vgl. Voss, »Filmerfahrung und Illusionsbildung«, S. 80; Vivian Carol Sobchack, Carnal Thoughts. Embodiment and Moving Image Culture, Berkeley, CA 2004, S. 53 ff.

86 Hantelmann, How to do things with art, S. 59.

87 Blümlinger, Kino aus zweiter Hand, S. 21 f. 


\section{Archive und Archäologie in der Kunst}

Erinnerung und Geschichte sind in der zeitgenössischen Kunst mit keinem anderen Thema so häufig in Verbindung gebracht worden wie mit dem Archiv. Spätestens seit dem Aufkommen von Collage, Assemblage und Montage in der Kunst der 1920er und 193oer Jahre gehört das Sammeln, Bewahren und Neukombinieren zur Arbeitsweise moderner Künstler/innen. Unter anderem Joseph Cornell, Christian Boltanski oder Marcel Broodthaers haben den Gedanken künstlerischer Sammlungs- und Ausstellungstätigkeit und die Schaffung individueller $>$ Museen $<$ im Rahmen der Konzeptkunst etabliert. ${ }^{88}$ Das Archiv dient dabei nicht, wie zum Beispiel das Depot eines Museums, der reinen Aufbewahrung von Kunst oder Materialien, welche für eine spätere Präsentation im Rahmen einer Ausstellung gedacht sind. Vielmehr wird es selbst als Erinnerungs- und Ordnungssystem ausgestellt. Ein Beispiel hierfür ist die Arbeit Top Secret (1989) des bulgarischen Künstlers Nedko Solakov auf der Documenta 12 (2007). Zwei hölzerne Karteikästen bewahren Zeugnisse der frühen Kollaboration des Künstlers mit dem bulgarischen Geheimdienst Intelligenzia auf. Der Besucher / die Besucherin kann diese Kästen durchsuchen und die Verweise, Spuren und Fotografien zu einer Chronik der Ereignisse zusammenfügen. Man kann, wie Monika Rieger beschreibt, zwischen zwei Arten der künstlerischen Archivarbeit unterscheiden: einerseits Künstler/ innen, die »archivische Prinzipien und Verfahren als Formprinzip verwenden«, anderseits Künstler/innen, die das Archiv in Verbindung zu den kulturwissenschaftlich geprägten Topoi von »Gedächtnis und Erinnerung, Kanonbildung oder Geschichtskonstruktion « thematisieren. ${ }^{89}$

In der Theoriebildung >zirkuliert< der Diskurs des Archivs über die Wissenschaftsgeschichte hinaus in verschiedensten Bereichen der Geistes- und Kulturwissenschaften seit einiger Zeit und hatte seinen Höhepunkt in den 199oer Jahren. Er basiert insbesondere auf den Ideen von Michel Foucault und Jacques

88 In Ausstellungen wie Deep storage. Arsenale der Erinnerung. Sammeln, Speichern, Archivieren in der Kunst (Haus der Kunst, München, 1997) oder Archive Fever. Uses of the Document in Contemporary Art (International Center of Photography, New York, 2008), aber auch in vielen Einzelausstellungen über geschichtlich arbeitende Künstler/innen, welche immer häufiger den Begriff des Archivs benutzen, wurde in den vergangenen Jahren das Archiv als Topos der zeitgenössischen Kunstproduktion und -reflexion diskutiert und etabliert.

89 Monika Rieger, »Anarchie im Archiv. Vom Künstler als Sammler«, in: Knut Ebeling, Stephan Günzel (Hg.), Archivologie. Theorien des Archivs in Wissenschaft, Medien und Künsten, Berlin 2009, S. 253-269, S. 257. 
Derrida zum Archiv. ${ }^{90}$ Foucault hat in seiner Archäologie des Wissens 1969 das Archiv als Grundlage der Möglichkeit von Wissen bezeichnet - das »Gesetz dessen, was gesagt werden kann «. ${ }^{91} \mathrm{Im}$ Kontext seiner poststrukturalistischen Geschichtstheorie stellt das Archiv ein Modell für >Aussagesysteme< dar. ${ }^{92}$ Mit dem Begriff >Archiv $<$ lehnt sich Foucault an den konkreten Ort der historischen Recherche an, darüber hinaus bedeutet Archiv jedoch generell die Möglichkeit der Bedingung von Wissen und von Aussagen, die auf Wissen basieren. Dass das Archiv »zum Schlüsselbegriff einer Geschichts- und Kulturtherorie« avancierte, beruht dabei auf dem Wandel, den das Bild von Vergangenheit durchlaufen hat: Nimmt man die Vergangenheit als Archiv wahr, kann man nicht mehr von einer Repräsentation, sondern nur von einer Produktion einer Ordnung von Vergangenheit ausgehen. ${ }^{93}$ Wie Knut Ebeling und Stephan Günzel ausführen, ist das Archiv zugleich »Institution wie Konzeption, Arbeitsort und Methode « ${ }^{94}$ In dieser Verschränkung von realem Ort, historischem und materiellem Objekt/Gegenstand und Praxis des (Er-)Forschens treffen sich wissenschaftshistorische und künstlerische Praktiken.

Welche Bedeutung haben nun der Begriff und das Konzept des Archivs, wie es von Foucault und Derrida ausgehend entwickelt wurde, für die zeitgenössische Kunst und im Speziellen für das bewegte Bild im Kunstkontext? In seinem Aufsatz »The Archival Impulse ${ }^{95}$ diskutiert der amerikanische Kunsthistoriker Hal Foster die unterschiedlichen Methoden verschiedener Künstler/ innen, darunter auch Tacita Dean, deren Werk im Kontext des Archivischen verortet werden muss. »Archival artists « machten verlorene oder verschobene historische Information zumeist in Installationen physisch präsent. Sie seien von historischen Bruchstücken angezogen, die einen neuen Anfang in eine andere Richtung erlaubten: »[They] are often drawn to unfulfilled beginnings or incomplete projects - in art and in history alike - that might offer points of departure again. ${ }^{96}$ Arbeit in einem archivischen Sinne sei dabei von dem allegorischen Impuls zu unterscheiden, den Craig Owens der

9o Einen Überblick über den Archivdiskurs bietet der Sammelband Archivologie. Theorien des Archivs in Wissenschaft, Medien und Künsten von Knut Ebeling und Stephan Günzel. Die Relevanz in der zeitgenössischen Kunst diskutiert u. a. Charles Merewether (Hg.), The Archive, London 2006.

91 Michel Foucault, Archäologie des Wissens, 13. Auflage, Frankfurt am Main 2007, S. 187.

$92 »[$ Das Archiv] ist das allgemeine System der Formation und Transformation der Aussagen.«, ebd., S. 188.

93 Knut Ebeling, Stephan Günzel, »Einleitung«, in: dies., Archivologie, S. 7-26, S. 14.

94 Ebd., S. 10.

95 Hal Foster, »An Archival Impulse«, in: October 110, Herbst 2004, S. 3-22.

96 Ebd., S. 5 . 
postmodernistischen Kunst zuschreibt. ${ }^{97}$ Es gehe nicht um Aneignung oder Nachahmung, sondern darum, Verbindungslinien zwischen geschichtlichen Fäden und der eigenen künstlerischen Existenz zu schaffen. Archivisches Interesse zeugt laut Foster von einer utopischen Ambition, hat aber auch eine andere Seite, eine paranoide Dimension. ${ }^{98}$

Betrachtet man die Arbeiten des schottischen Künstlers Douglas Gordon und der britischen Künstlerin Tacita Dean, kann man eine Vermischung der beiden oben angesprochenen, von Monika Rieger unterschiedenen Typen künstlerischer Archivarbeit beobachten. Beide bedienen sich archivischer Praktiken wie Sammlungen und Listen, um Materialien und Daten zu ordnen und zeitliche wie räumliche Konstellationen der Wahrnehmung zu erzeugen, so zum Beispiel Gordons List of Names (seit 1989 fortlaufend) oder Deans Floh (2002). Andererseits arbeiten sie mit und in Archiven, Flohmärkten oder anderen Sammlungen, um Material aufzuspüren, welches später die Grundlage eigener Arbeiten darstellt. Beispiele hierfür sind die Found-Footage-Arbeiten von Gordon, die Fotoarbeit Floh, in der Dean auf dem Flohmarkt gefundene Bilder zu einem neuen Buchprojekt gebündelt hat, oder ihre frühen Arbeiten, die auf Zeitungsausschnitten und Fotografien beruhen, wie Girl Stoaway (1994) und Dissapearance at Sea I und II (1996) oder Section Cinéma (2002), der Film über ein ehemaliges Studio von Marcel Broodthaers. Somit setzt sich ein Prozess der Aneignung und der Reverenz an bestimmte Themenkomplexe und Geschichten in Gang. Das Interesse für einzelne Schicksale, Geschichten und Legenden trifft hierbei auf die übergeordnete Frage, wie sich geschichtliche Prozesse konstituieren, wie die Relevanz des einzelnen Menschen, Ereignisses, Dokuments im Verhältnis zur Konstruktion von Geschichte zu beurteilen ist.

Im Weiteren soll nun zunächst die >archäologische< Arbeit von Douglas Gordon mit Found Footage diskutiert werden, wobei ein Vergleich mit einem Werk der deutschen Künstler Matthias Müller und Christoph Girardet die verschiedenen Ansätze und spezifischen Charakteristika videotechnischer Found-Footage-Arbeiten verdeutlicht.

\section{Hysterie, Trauma und Erinnerung}

Der Schotte Douglas Gordon gehört zu den Hauptvertretern einer neueren Filmkunstbewegung, die im Kontext der Young British Artists insbesondere

\footnotetext{
97 Craig Owens, »The Allegorical Impulse. Toward a Theory of Postmodernism«, in: October 12, Frühjahr 1980, S. 67-86.

98 Foster, »An Archival Impulse«, S. 22.
} 
im englischsprachigen Raum seit Mitte der 199oer Jahre aufgekommen ist. 1966 geboren, wurde Gordon 1993 mit seiner Arbeit 24 Hour Psycho, welche im Tramway in Glasgow und in dem Ausstellungshaus Kunst-Werke Berlin e. V. gezeigt wurde, international bekannt. Von Beginn an arbeitete Gordon in verschiedenen Medien, den Hauptkomplex seines CEuvres machen jedoch Videoarbeiten, Fotografien und konzeptuelle Textarbeiten aus. Zu den FoundFootage-Werken der 199oer Jahre zählen einerseits Adaptionen und Transformationen von klassischen Hollywoodfilmen, andererseits die Arbeit mit frühen medizinischen Lehrfilmen. Mit Beginn der 2oooer Jahre ändert sich Gordons Arbeitsweise, er filmt nun größtenteils selbst und widmet sich dabei so unterschiedlichen Themen wie dem Fußball, der Performance oder der klassischen Musik. Nachdem im Kapitel 1 bereits die Spielfilmadaptionen von Psycho in 24 Hour Psycho (1993) und von The Searchers in der Arbeit 5 Year DriveBy (1995) besprochen wurden, soll im Folgenden die Aneignung medizinischer Lehrfilme in drei Arbeiten Gordons einer genaueren Analyse unterzogen werden. In ihnen werden ebenfalls Verlangsamung und Wiederholung als Stilmittel eingesetzt, jedoch eröffnen sie mit der Adaption pädagogischdokumentarischer Filme ein neues Feld. Indem Material verwendet wird, das lange vor der Normierung der kommerziellen Filmform durch das >System< Hollywood zu Lehrzwecken erstellt wurde, kann daran einerseits die Unterscheidung des Fiktionalen und des Dokumentarischen diskutiert, andererseits der Bezug zum »Kino der Attraktionen« der Frühzeit des Films hergestellt werden.

Die drei Arbeiten 10ms ${ }^{-1}$ (1994), Trigger Finger (1994) und Hysterical (1994-1995) bilden neben den Hollywoodfilm-Adaptionen eine geschlossene Werkgruppe innerhalb von Douglas Gordons CEuvre, da sie sich auf Filmmaterial ähnlichen Ursprungs beziehen. Alle drei behandeln das Thema der Hysterie, ihre körperlichen Symptome, die visuelle Repräsentation im Kontext medizinischer Lehre und die Behandlungsmethode.

\section{Hysterical (1994-1995)}

Hysterical besteht aus zwei Schwarz-Weiß-Videoprojektionen auf zwei freistehenden, leicht nach hinten geneigten Leinwänden, die in einem stumpfen Winkel zueinander aufgestellt sind (Abb. 10). Auf eine Leinwand wird ein Schwarz-Weiß-Film als Video projiziert, auf der anderen ist der gleiche Film in Zeitlupe zu sehen. In manchen Ausstellungssituationen wird der Film auf die Rückseite der zweiten Leinwand projiziert, so dass der verlangsamte Film gespiegelt erscheint. Die Aufnahme läuft im Loop, eine Sequenz hat ca. 3 Minuten. In der Bildmitte wird eine maskierte Frau mit hochgesteckten dunklen Haaren im hochgeschlossenen schwarzen Kleid gezeigt, die zwischen 


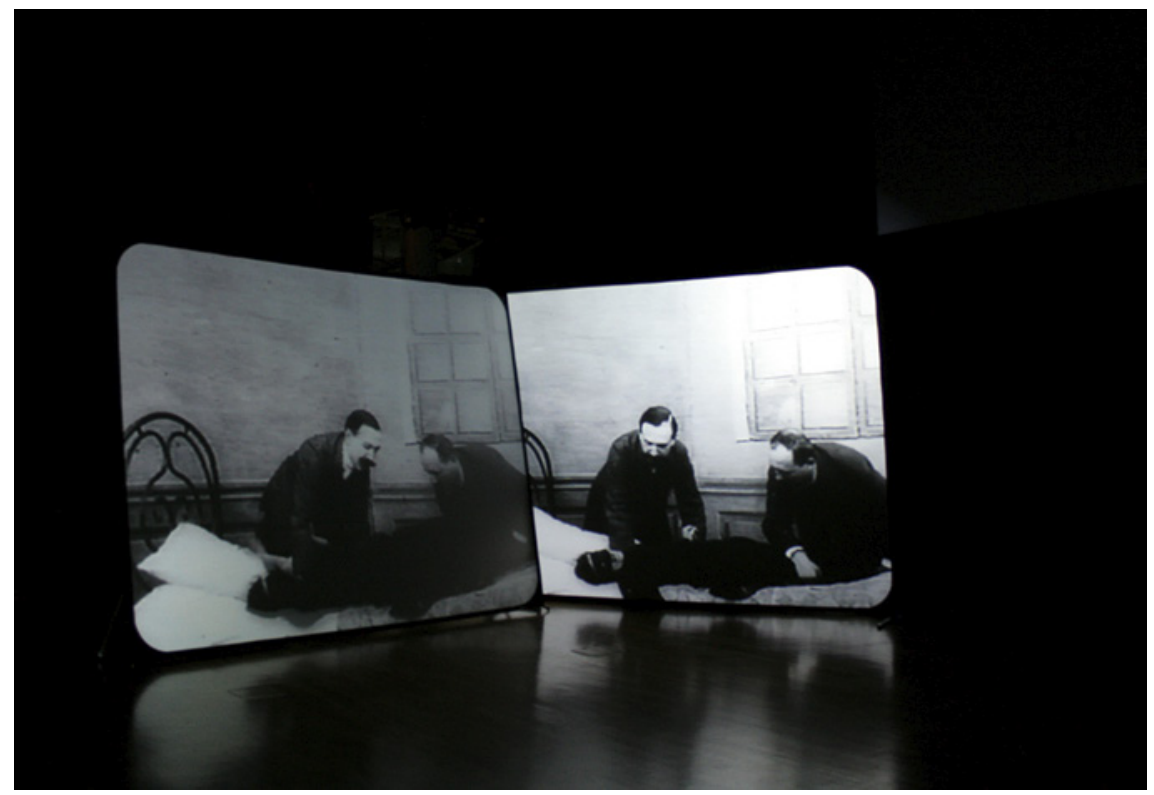

Abb. 10

Douglas Gordon, Hysterical, 1994-1995, Ausstellungsansicht Kunstmuseum Wolfsburg, 2007

zwei mit Anzügen bekleideten Männern steht. Zu Beginn des Films ist sie mit dem älteren Mann im Gespräch. Hinter der Gruppe steht ein Bett, dahinter sieht man die Wand und einen angedeuteten Fensterrahmen. Die Frau fasst sich mehrmals an den Hals und an den Bauch und erzählt dem Mann dabei etwas, der Film ist jedoch stumm. Mitten in ihrer Rede fällt sie nach einigen Momenten schlagartig hinten über und wird von den Männern auf das bereitstehende Bett gelegt. Sie halten ihre zuckenden, krampfenden Arme und Beine fest und tragen dabei Sorge, dass der Körper für die Kamera stets sichtbar bleibt. Sobald sie die Glieder mit sicherem Griff fixiert haben, geht die krampfartige Bewegung auf den Unterleib der Frau über. Nachdem sie sich beruhigt hat, blickt der ältere Mann mit einem verschmitzten Grinsen zur Kamera. Er drückt rhythmisch auf den Unterleib der Frau, worauf zwei weitere Anfälle folgen. Diese können mit gezielter Fixierung >gelindert< werden und werden dadurch unterbrochen, dass der Mann in Richtung Kamera spricht, wohl um eine Rückmeldung zu bekommen, ob man noch weiterfilmen kann.

Der Betrachter oder die Betrachterin sieht sich hier - im Gegensatz zu Gordons früherer Arbeit 24 Hour Psycho, in der er mit Hitchcocks Psycho einen bekannten Spielfilm verarbeitet - einer unbekannten Szene gegenüber. Die Bildästhetik - Aufnahmequalität, Textur und Störungen des Materials, Licht, die Frisuren und Kleidung der Protagonisten - lässt hinsichtlich der 
Entstehungsbedingungen des Film Footages auf die Frühzeit des Kinos schließen, das geschlossene Setting erinnert an die Anfang des 20. Jahrhunderts gängige Praxis, theaterähnliche Vorführungen mit einer statischen Kamera abzufilmen. Doch nicht nur die Ästhetik des Materials und Inventars, sondern auch die Zurschaustellung dieser Abfolge merkwürdiger Ereignisse ist heutigen Betrachter/innen fremd. Es scheint fast unmöglich, hier von dokumentarischem oder fiktionalem Film zu sprechen; auf der einen Seite wirken der Anfall und die Reaktion darauf genau geplant: Kadrierung, Setting und Timing sprechen dafür, dass hier ein Schauspiel aufgeführt wird, in dem die maskierte Frau und die Männer klare Rollen einnehmen. Auf der anderen Seite durchkreuzt die Kommunikation des Älteren mit dem Kameramann immer wieder die Präsentation des Anfalls als Ereignis. Der direkte Blick in die Kamera ist ein charakteristisches Erbe des frühen Kinos, dem dieser Film, wenngleich einige Jahre später entstanden, noch verhaftet ist. Dieses Kino vor und um 1906 bezeichnete Tom Gunning 1986 als »Kino der Attraktionen«. Gunning leitete mit seiner Analyse, dass das frühe Kino gerade nicht dem narrativen Impuls des wenig später florierenden Kinos eines D. W. Griffith entspricht, eine Wende in der Filmgeschichtsschreibung und Medienarchäologie ein. ${ }^{99}$ Seine Einschätzung beruht auf der Tatsache, dass das Kino in seinen Anfängen auf die Schausteller- und Spektakelkultur der Jahrmärkte und Varietétheater zurückgeht, wo die Kinematografie ihr erstes Publikum fand. Im Rahmen dieser Spektakelkultur ging es weniger um die Erzählung von Geschichten, sondern standen die technischen Gerätschaften, die erzeugte Bewegungsillusion und manipulierende Tricks mittels Montage, Rückwärtslauf oder Zeitlupe im Mittelpunkt des Interesses. Darüber hinaus erregten Szenen, die dem Blick sonst verborgen blieben (wie erotische Nahaufnahmen von Frauen) die »visuelle Neugier« des Publikums. ${ }^{100}$ Der Blick in die Kamera, der die Illusion eines einheitlichen filmischen Universums zerstört, ist darin nicht verboten, sondern ausdrücklich erwünscht: Vor der Funktion der Erzählung und dem Realismuseffekt kommt diesem Kino zunächst einmal die Faszination zu, dass sich etwas zeigt - es ist Gunning zufolge ein »exhibitionistisches« Kino, in dem der Kontakt zum Publikum ausdrücklich erwünscht sei.101

99 Das Konzept des »Cinema of Attractions« wurde nicht nur zum vielzitierten Begriff innerhalb der Forschung über frühen Film, sondern beispielsweise auch auf Slapstick, Experimentalfilm, Horrorfilm und Youtube übertragen. Die anhaltende Bedeutung von Gunnings Ansatz für die Forschung unterstreicht der Sammelband von Wanda Streuven (Hg.), The Cinema of Attractions Reloaded, Amsterdam 2006.

100 Vgl. Tom Gunning, »Das Kino der Attraktionen. Der frühe Film, seine Zuschauer und die Avantgarde«, in: Meteor 4, 1996, S. 25-34, S. 29.

101 Ebd., S. 27. 
Gordon stellt den Film buchstäblich aus: Wie ein Objekt präsentiert er ihn in erweiterter Sichtbarkeit, indem er ihn auf dem freistehenden, transparenten Träger zeigt und ihm sein verlangsamtes Double zur Seite stellt. Sein Vorgehen hat er selbst einmal als »archäologische Forschungsarbeit« beschrieben: Er grabe altes Material aus, entstaube es und bringe so Verborgenes zum Vorschein. ${ }^{102}$ Diese Prozedur der Freilegung verborgener Sinnschichten funktioniert auf einer doppelten Ebene: Zunächst wird ein Stück Film, das auf eine bestimmte Art und Weise seine Aufmerksamkeit erregt, isoliert und aus dem Filmarchiv in den Ausstellungskontext geholt. Diese Auswahl ist höchst selektiv und subjektiv und verweist auf die Frage nach der Autorschaft. Dann nimmt er in der Präsentation mehrere entscheidende Änderungen vor: Er verwendet ein anderes technisches Medium, projiziert den Film auf eine freistehende, transparente Leinwand und zeigt ihn, wie bei Hysterical, als Doppelprojektion in zwei Geschwindigkeiten. Das filmische >Objekt erfährt institutionelle und mediale Verschiebungen, die seine Sichtbarkeit - und die damit verbundene Sinngenerierung - gleichzeitig erhöhen und verstellen: Wir sehen das filmische Detail vergrößert, verlangsamt und im Loop; wir können durch die Doppelung verschiedene Szenen miteinander vergleichen; wir können uns der Leinwand nähern, sie umkreisen, andere Blickwinkel darauf gewinnen; durch die strikte Kappung aller Informationslinien über Gegenstand, Herkunft, Entstehungszeit des Footages wird in einem musealen Raum ein forschender Blick auf das Material selbst provoziert. Dieser Blick bemerkt bereits vor jeder deutenden Interpretation das inszenierte Setting des Films, die weisenden Gesten und die heute fremd wirkenden Blicke in Richtung Kamera; in diesem Kontext kann der Anfall der Frau nicht als unvorhergesehenes Ereignis, sondern nur als Teil einer genau getimten Dramaturgie wahrgenommen werden, wobei auch die geschlechterspezifische Rollenverteilung auffällt. Dennoch kann man die bildliche Sichtbarmachung nicht mit einer sachlichen präikonografischen Beschreibung gleichsetzen, denn Gordon liefert mit dem Titel einen entscheidenden Hinweis auf das Ausgangsmaterial: Er verweist auf das Thema des Films und schafft zugleich einen Kontext, nämlich die Darstellung der Hysterie in frühen Medizinlehrfilmen. Wie dort mithilfe der Vorführung von Einzelfällen Symptome der Krankheit exemplarisch gezeigt werden, so zeigt auch Gordon ein berühmtes Einzelwerk, ohne es zu identifizieren. In diesem wird die Hysterie, wie bereits seit der Antike überliefert und auch etymologisch an der Ableitung der Bezeichnung von hystéra (gr. Gebärmutter) erkennbar ist, als weibliches Leiden präsentiert, das durch die angebliche Wanderung der Gebärmutter im Körper ausgelöst werde.

102 »Vibration - Found Footage«, 9.4.2003, http://www.arte.tv/de/Kultur-entdecken/tracks/ Sendung-vom-11--April-2003/391870,CmC=391896.html [12.10.2015]. 
Nur durch gezielte Behandlung des Unterleibs der Frau, durch Druck oder Stimulation, könne, so die allgemeine Lehrmeinung bis ins 20. Jahrhundert hinein, das nervöse Leiden Linderung erfahren, wie ja auch der Originalfilm des bekannten italienischen Nervenarztes Alberto Negro und des renommierten Kameramanns Roberto Omegna aus dem Jahr 1908 belegt. ${ }^{103}$ Der Film erlangte innerhalb von zwei Diskursen Bedeutung: zum einen in der zeitgenössischen Medizin als Lehrfilm, zum anderen in der Filmgeschichte, wo er als erster Film gilt, der nicht nur eine wissenschaftliche Diagnose illustrierte, sondern der Anamnese diente. ${ }^{104}$ All dies verschweigt Gordon und öffnet dadurch den Film für neue Interpretationen, die durch die aktive Eliminierung der diskursiven Vorcodierung ebenso wie durch die historische Distanz ermöglicht werden.

\section{Autorschaft}

Die Frage nach der Autorschaft ist in Douglas Gordons Werk zentral und kommt nicht nur in seinen Found-Footage-Arbeiten, sondern auch in den Video- und Textarbeiten zum Ausdruck. Er lanciert häufig offene Verweise auf bekannte historische Film- und Videoarbeiten, ${ }^{105}$ lässt unklare Katalogtexte publizieren, ${ }^{106}$ beschreibt seine Found-Footage-Arbeiten als Do-it-yourselfProdukte und streut zum Teil gezielt falsche Informationen über die Herkunft oder Inspiration von Bildern.

103 Die Repräsentation von Hysterie hat ihre Wurzeln in der Malerei des 18. Jahrhunderts bei Gericault und Goya sowie in der fotografischen Praxis am Pariser Hôpital de la Salpêtrière, worauf weiter unten näher eingegangen wird.

104 Vgl. Ute Holl, »Neuropathologie als filmische Inszenierung«, in: Martina Hessler (Hg.), Konstruierte Sichtbarkeiten. Wissenschafts- und Technikbilder seit der Frühen Neuzeit, München 2006, S. 217-240, S. 222. Der Film ist unter dem Titel La nevropatologia bekannt. Ursprünglich 108 Minuten lang, kursieren verschiedene wenige Minuten lange Ausschnitte auf Kompilationen beziehungsweise filmarchivarischen Webseiten.

105 Diese offenkundigen Verweise zeigen sich nicht nur in der Aneignung in Form von Wiederverwendung, sondern auch durch Abfilmen von Projektionen wie beispielsweise Bootleg: Empire, 1995, wo Gordon den Avantgardefilm Empire von Andy Warhol abgefilmt, geschnitten und wieder aufgeführt hat. Weitere Verweise zu Ikonen der Film- und Videogeschichte finden sich in den Textarbeiten, die meist über die Wände des Ausstellungsraums verteilt sind, sowie in den Videoarbeiten, die auf Monitoren gezeigt werden. Die Arbeit The right hand doesn't care what the left hand isn't doing kann beispielsweise als Antwort auf Gary Hills Text And if the Right Hand did not know what the Left Hand is doing von 1990 gedeutet werden, in dem dieser über die Geschichte der Videokunst sinniert. Auch die Gegenüberstellung zweier ineinander übergehender Texte findet bei Gordon ein Echo, so dass die Verweise als bewusste historische Referenz gesehen werden können.

106 Im Katalog zur Ausstellung Kidnapping 1998 im Stedelijk Van Abbemuseum Eindhoven wurde ein Brief über den Künstler von seinem Bruder, David Gordon, veröffentlicht. Im Katalog des Kunstvereins Hannover, ebenfalls 1998, wurde eine verrätselte Kurzbiografie »von einem Freund« veröffentlicht. Beide Texte weisen eine andere Person als Autor aus. 
Gordon spielt bewusst mit der postmodernen Aufhebung der Kategorie des Autors, sein eigenes künstlerisches Handeln sieht er als die Funktion eines Schnittmeisters, der Ideen und Material sortiert und neu arrangiert, um beim Publikum Denkprozesse anzustoßen. ${ }^{107}$ Indem er den Akt der Aneignung und Neukontextualisierung an die erste Stelle setzt und nicht die Vergegenwärtigung des Dargestellten oder dessen Symbolik, kann hier jedoch auch von einer Readymade-Strategie gesprochen werden: Beim Readymade geht es nicht in erster Linie darum, etwas Nichtkünstlerisches zum Kunstwerk zu erklären - denn bei Found Footage handelt es sich nicht um Gebrauchsgegenstände, sondern um bereits semantisch codierte ästhetische Produkte -, sondern um die zeitliche Setzung einer künstlerischen Idee, wie Sebastian Egenhofer beschreibt:

Die Zeit, und das heißt ein irreduzibles Maß an Kontingenz, ist das Element, in dem eine vom Künstler nie ganz kontrollierte Setzung zum Werk erst nachträglich wird. Duchamp hat diese Struktur des Werdens des Werks mit dem Readymade formalisiert, das die Funktion der Autorschaft auf den Akt der Datierung, der Entscheidung über den Anfang seiner Existenz in der Zeit zuspitzt [...]. ${ }^{108}$

Gordons Setzung des Films in Kunstraum ist paradigmatisch für den Umgang mit historischem Material in der Kunst der 199oer Jahre, weil sie in ihrer Geschichtsbezüglichkeit uneindeutig bleibt. Auf der einen Seite entledigt er den Film aller interpretativen Bezüge und bietet mit seiner Installation sozusagen die voreingestellte Apparatur zur Analyse des unerwarteten Materials durch die Betrachter/innen; auf der anderen Seite zeugt die Bearbeitung gerade dieses speziellen Films von Nero und Omegna vom Bewusstsein für eine »kulturelle« Historizität, wie Christa Blümlinger sie in Werken beschreibt, die »auf symbolische Formen, wahrnehmungsgeschichtliche Zusammenhänge oder auch Bedingungen historischer Diskursivität abzielen «.109 Die neuropathologischen Filme sind mediale Figurationen körperlicher Leiden, die wiederum als Ausdruck psychischer Störungen oder Traumata sichtbar werden. Auf das symbiotische Verhältnis, das zwischen den

\footnotetext{
107 "You could call it recontextualisation, or you could call it synthesis, or perhaps you could call it editing. It's funny, in some ways I'd feel more confident to say that I am a good editor rather than a >good artist.« (Douglas Gordon, Jan Debbaut, »... in conversation. jan debbaut and douglas gordon«, in: Stedelijk Van Abbemuseum, Eindhoven, Douglas Gordon. Kidnapping, Ausstellungskatalog Stedelijk Van Abbemuseum, Eindhoven 1998, S. 11-55, S. 39.)

108 Sebastian Egenhofer, Abstraktion, Kapitalismus, Subjektivität. Die Wahrheitsfunktion des Werks in der Moderne, München 2008, S. 95.

109 Blümlinger, Kino aus zweiter Hand, S. 10.
} 
Ärzten, den Patienten bzw. Patientinnen und den bilderzeugenden Techniken herrschte, wird später zurückzukommen sein. Für den hier verwendeten Film hat dies Ute Holl tiefgehend analysiert. ${ }^{110}$

\section{Shell shock - Konvergenzen körperlicher und medialer Dysfunktion in 10ms $^{-1}$ (1994) und Trigger Finger (1994)}

Das Filmmaterial, auf das Douglas Gordon in den ähnlichen Werken roms ${ }^{-1}$ (Abb. 11) und Trigger Finger (Abb. 12) zurückgreift, entstand im Netley Hospital, einem großen ehemaligen Militärkrankenhaus nahe Southampton in Großbritannien, wo während des Ersten und Zweiten Weltkriegs tausende verwundete britische und amerikanische Soldaten behandelt wurden. 1917 und 1918 wurden dort zahlreiche Fälle des sogenannten shell shock Syndroms behandelt, einer posttraumatischen Belastungsstörung, welche durch die Teilnahme an Kriegshandlungen ausgelöst wurde. Der Name leitet sich aus der Vermutung ab, die psychischen und neurologischen Symptome der Patienten rührten von der Druckwelle der Explosionen von Granaten her und es gebe somit einen mechanischen Grund für die Störungen wie beispielsweise zu große Erschütterung. In den kurzen Filmen, die über das British Pathé Film Archive öffentlich zugänglich sind und vermutlich dort von Gordon gefunden wurden, ${ }^{111}$ werden die Heilungserfolge an den Soldaten dargestellt und ihre mögliche Wiedereingliederung in die Gesellschaft und den Militärdienst in Aussicht gestellt. Jedem Patienten sind hier eine bis drei Minuten gewidmet, wobei die Präsentation stets nach dem gleichen Muster abläuft: In einem Zwischentext wird der Soldat mit Dienstgrad vorgestellt und sein Leiden beschrieben, teilweise aber auch nur die Diagnose genannt. Die Beschwerden sind häufig ein nervöses Zittern der Beine, Probleme mit dem Laufen und Halten des Gleichgewichts (Ataxie), unkontrollierbare Bewegung und irrationale Ängste vor bestimmten Geräuschen oder Gegenständen. Dann wird die Therapie beschrieben, welche in allen Fällen erfolgreich verläuft. Das dazugehörige Filmmaterial zeigt den kranken Patienten im Krankenbett, Krankenzimmer oder im Hof des Hospitals, seine körperlichen Symptome

\footnotetext{
110 Vgl. Holl, »Neuropathologie als filmische Inszenierung«.

111 In geringer Auflösung sind die verschiedenen Rollen über die Webseite des British Pathé Film Archive anzusehen. Der hier verwendete Film findet sich auf »War Neuroses Version B Reel 2 1917-1918«, https://www.britishpathe.com/video/war-neuroses-version-b-reel-2 [26.4.2020] ab Minute 6:20. Das Material von Trigger Finger beginnt bei Minute 10:0o auf derselben Rolle. Nähere Informationen zu den gezeigten Szenen bietet darüber hinaus die medizinhistorische Wellcome Library: »War Neuroses. Netley Hospital,1917«, Wellcome Library, Wellcome Trust, 2008, http://catalogue.wellcomelibrary.org/record=b1667864 S3 [26.4.2020]. Bei Sachinformationen beziehe ich mich im Weiteren auf diese Webseite.
} 


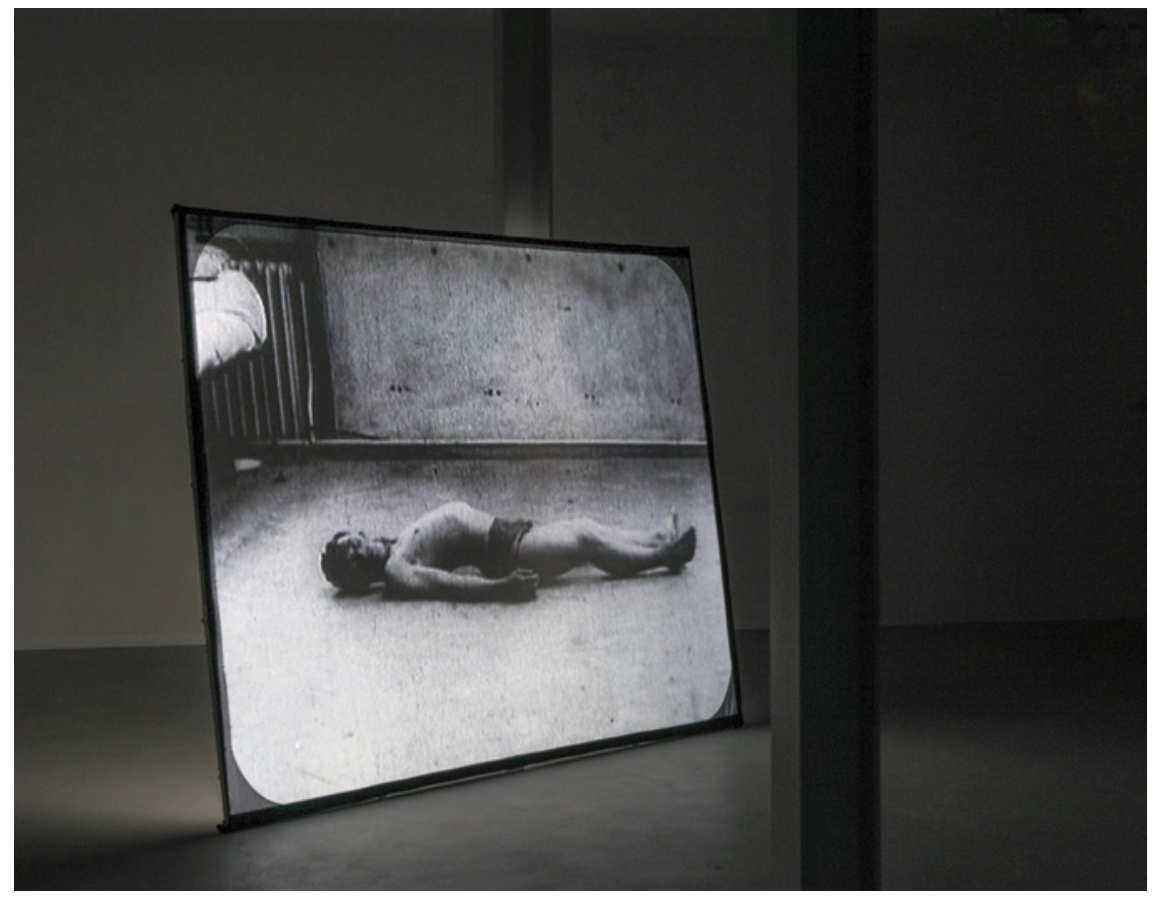

Abb. 11 Douglas Gordon, $1 \mathrm{oms}^{-1}$, 1994, Ausstellungsansicht Dox, Prag, 2009

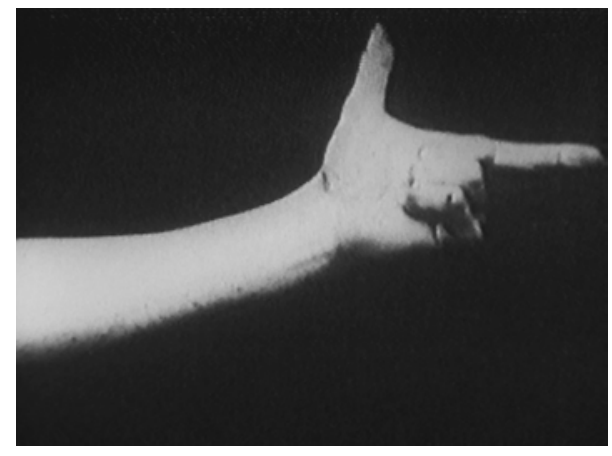

Abb. 12

Douglas Gordon, Trigger Finger, 1994

präsentierend. Dann sieht man in einigen Fällen ein Zwischenstadium, entweder während der Behandlung oder mit bereits erzielten Verbesserungen, und schließlich den nahezu oder vollständig genesenen Patienten, der entweder im Hof des Krankenhauses seine Fähigkeiten demonstriert oder bereits wieder einer zivilen Arbeit nachgeht. Am Schluss des Films wird von Patienten eine Schlacht im Feld nachgespielt.

Wurde bei der Analyse von Hysterical zunächst vom Kunstwerk selbst ausgegangen, um dann auf das Ausgangsmaterial zu kommen, soll hier der 
umgekehrte Weg eingeschlagen werden. Schon im Ausgangsmaterial ist nämlich das Verhältnis von Dargestelltem und Darstellungsweise prekär, filmische Form und medizinischer Inhalt greifen auf der Ebene des bewegten Bildes stark ineinander, was schließlich von Gordon für seine Videoarbeit aufgegriffen wird. Ähnlich wie der Lehrfilm über Hysterie von Camillo Negro handelt es sich bei $10 \mathrm{~ms}^{-1}$ und Trigger Finger um zu Unterrichtszwecken erstelltes Material, dessen Entstehung in den Jahren 1917 oder 1918 jedoch im militärischen Zusammenhang des Ersten Weltkriegs steht: Die Truppenärzte sahen sich plötzlich einer hohen Zahl psychisch versehrter Soldaten und deren körperlichen Leiden gegenüber und waren von der Anzahl und Schwere der Fälle überwältigt und überfordert; zugleich standen sie jedoch unter dem hohen Druck, diese schnellstmöglich wieder kampffähig zu machen - eine logistische und ethisch gleichermaßen belastende Herausforderung.

Es wurden bei der Erforschung, Vermittlung und Therapie der neuen Krankheitsbilder zur Dokumentation und Schulung verschiedene mediale Formen eingesetzt, neben Schrift und Fotografie insbesondere auch die Kinematografie, wie Julia Köhne in ihrer Studie zu den sogenannten Kriegshysterikern darlegt. ${ }^{112}$ Die visuellen Repräsentationen dienten der Dokumentation, der Verbreitung und der Evidenz der Heilung, aber auch der Entlarvung von Simulanten:

\begin{abstract}
Das Sichtbarmachen sollte vor allem dadurch möglich sein, dass die filmisch aufgenommene Bewegung eingehend und wiederholt studiert und damit auf ihre >Echtheit` geprüft werden konnte. Der Film als Medium der Täuschung und Simulation sollte den täuschenden Kriegshysteriker entlarven helfen; letzterer sollte also mit seinen eigenen Mitteln, nämlich denen der (filmischen) Simulation, geschlagen werden. ${ }^{113}$
\end{abstract}

Die Filme wurden zur Wissensproduktion eingesetzt, das Medium war dafür aber, wie sich zeigen sollte, nur bedingt geeignet. Köhne beschreibt, wie die technischen Eigenheiten des frühen Films - das Zittern und Ruckeln des Bildes, die Unschärfen und Belichtungsschwierigkeiten, die Sprünge und zeitlichen Variationen aufgrund der handbetriebenen Kurbel - und die Bewegungsmuster der Kriegshysteriker korrelieren, wie sich das eine im anderen spiegelt und so die Dysfunktionalität beider Systeme, der filmischen Darstellung wie der medizinischen Wissensproduktion, offenlegen:

\footnotetext{
112 Julia Barbara Köhne, Kriegshysteriker. Strategische Bilder und mediale Techniken militärpsychiatrischen Wissens (1914-1920), Husum 2009. Der Ausdruck Kriegshysteriker ist insofern interessant, als ein vormals ausschließlich weiblich konnotiertes diffuses Krankheitsbild neurologischer und psychiatrischer Auffälligkeiten hier erstmals auf männliche Patienten angewendet wurde.

113 Ebd., S. 187.
} 
Die filmische Aufnahme der kriegshysterischen Bewegung brachte das Zusammenspiel dieser fein aufeinander abgestimmten Faktoren Zeit, Bewegung, Film durcheinander. Die technische Insuffizienz verhinderte, dass die Darstellung lückenlos vonstatten ging. Der motilitätsgestörte Kriegszitterer machte das dem Medium damals inhärente Zitterphänomen sichtbar und bildete auf diese Weise die Grenzen der neuen Medientechnik ab. Der Hysteriker mit seinen aus der Normalzeit gefallenen Körperbewegungen gab ein anderes Zeitmass an. ${ }^{114}$

Die bereits zur Zeit der Erstellung der Filme thematisierte Problematik, dass der Film mit seinen ihm eigenen Bewegungen und seiner zeitlichen Variabilität als Medium sichtbar bleibt, also nicht genug Transparenz aufweist, um hinter die Darstellung körperlicher Bewegungen zurückzutreten, macht Gordon zum Thema seiner videografischen Adaptionen. Er stellt dabei die ruckartige Bewegung, Verlangsamung und Wiederholung visuell in den Vordergrund und kommentiert so den Konflikt, den Köhne anspricht, mit bildlichen Mitteln. Der Film, aus dem Gordon für seine Videoarbeit $10 \mathrm{~ms}^{-1}$ einen Ausschnitt ausgewählt hat, zeigt einen unbekannten Soldaten, der, nur mit einer Art Lendenschurz bekleidet, in einem Krankenzimmer seine motorische Störung - einen stark verbogenen Rücken, der ihn in seinen Bewegungen einschränkt und nur steif und ataktisch laufen lässt - vorzuführen. Er wird mit folgendem Text angekündigt: »Hysterical pseudo-pseudohypertrophic muscular paralysis. (a) Before treatment. (b) An hour later; after treatment. (c) A week later.« In der ersten Sequenz läuft der Patient im Kreis durch den Raum zwischen Bettgestellen umher, die offensichtlich für die Filmaufnahmen zur Seite geschoben wurden. Er bleibt stehen und wendet sich der Kamera zu, eventuell weitere Anweisungen erhaltend. Nach einem Schnitt wird der Boden des Zimmers gezeigt, auf den sich der Patient fallen lässt. Er legt sich auf den Rücken, dreht den Kopf mehrfach nach rechts und links und steht wieder auf. Dies gelingt ihm aufgrund der Blockade des Rückens nur schwer und erforderte mehrere Versuche. Er kann sich schließlich nur durch eine starke, schwungvolle Drehung aus der Rückenlage befreien und läuft in unkontrollierten, abgehackten Schritten aus dem Bild. Im zweiten Abschnitt des Films, laut Zwischentitel eine Stunde später, sind deutliche Fortschritte zu erkennen. Die Bewegungen sind weicher und kontrollierter, und es gelingt ihm bereits beim ersten Mal, wieder aufzustehen. Die Aufnahme eine Woche später findet auf einem Dach statt, der Patient trägt Uniform und vollführt dieselbe Übung ohne Anzeichen motorischer Einschränkungen.

114 Ebd., S. 195. 
Gordon beschränkt sich auf einen Ausschnitt des Films, nämlich die Sequenz zu Beginn, als der Soldat sich zu Boden fallen lässt und nach mehreren vergeblichen Versuchen wieder aufsteht. In einer Videoversion wird das filmische Fragment auf eine freistehende, ca. 3 x 4 Meter große und durchscheinende Leinwand projiziert. Die Abspielgeschwindigkeit ist stark reduziert, so dass die Bewegungen sehr ruckartig aussehen, durch die Verlangsamung dehnt sich das wenige Sekunden dauernde Fragment auf etwa 10 Minuten.

Das Material von Trigger Finger findet sich nur kurze Zeit später auf derselben Filmrolle und wird mit folgendem Text angekündigt: »Hysterical contracture of hand persisting 35 months after wound near elbow«. Man sieht einen Unterarm und die erwähnte ausgestreckte Hand vor dunklem Hintergrund. Daumen und Zeigefinger sind abgespreizt, die anderen Finger weisen eine starke Kontraktion auf. Der Patient schüttelt die Hand zum Lösen der Kontraktion, ein Assistent hilft dabei. »Cured after 1/2-hour's treatment. Depressions in palm caused by pressure of nails now visible.« Auch hier wird nicht erläutert, wie die Heilung erreicht wurde, das Filmmaterial zeigt lediglich den Vorher-nachher-Effekt, dass die lang anhaltende Kontraktion nun behoben ist. Als Beweis des Krankheitsbildes werden die Druckwunden auf der Handinnenfläche präsentiert. Gordons Interesse an diesem Ausschnitt rührte wohl aus der ungewöhnlichen Haltung der Hand, welche unverkennbar der Geste gleicht, die allgemein als Simulation einer Schusswaffe verständlich ist - im Kinderspiel ebenso wie als Drohgebärde. So erklärt sich der Titel Trigger Finger, was einerseits Zeigefinger heißt, andererseits aufgrund der visuellen Nähe zum ausgestreckten Finger am Abzugshahn einer Waffe den Abzug einer Schusswaffe bezeichnet.

\section{Hysterie und Trauma}

Wie nun eignet sich Douglas Gordon diese bereits im Original bewegenden Dokumente, die Visualisierungen körperlichen und seelischen Leidens an? Wie verändert sich die Wirkung der Bilder durch die Dekontextualisierung, und was bedeutet dies für die Evidenz und Historizität der Bilder? Welche Rückschlüsse können wir aus Gordons Adaptionen für die Rolle des Found Footage in der Medienkunst der 199oer Jahre, ihren Umgang mit Geschichte und Erinnerung ziehen? Gordons zeitbasierte Bearbeitung erreicht, so soll hier schon einmal vorweggreifend festgestellt werden, mittels des zentralen Prinzips der Wiederholung eine visuelle Entsprechung der Zeitstruktur des Traumas in der Form der kinematografischen Installation ebenso wie in der Arbeit mit gefundenem Material.

Alle drei Werke verwenden Filmmaterial, in dem die Vergangenheit in Form von Erinnerungen ins Bild tritt und als neuropathologische Störung betrachtet 
und behandelt wird; diese Störung äußern sich in Figurationen körperlicher Leiden, für deren Behandlung die Aufzeichnung auf Film eine wesentliche Rolle spielt. In positivistischer Manier werden Symptome visualisiert und Fortschritte dokumentiert, zum Teil bis hin zur vermeintlichen Heilung. Dass die Erkrankungen psychische Ursachen haben, die weibliche Hysterie genauso wie die Symptome der Kriegszitterer auf seelische Traumata zurückzuführen sind, deren Verdrängung in den Bildern der Körper wiederkehrt, ist für die behandelnden Ärzte kein zureichendes Erklärungsmodell. Dabei muss zwischen den beiden Filmen der Kriegszitterer einerseits und dem Hysteriefilm andererseits unterschieden werden, und dies nicht nur aufgrund der unterschiedlichen Kontexte und Entstehungszeitpunkte. Die Hysterie hat ihren Ursprung als Krankheitsbild in den Räumen der Pariser Salpetrière, wo Jean-Martin Charcot sie mithilfe der Fotografie von Albert Londe untersuchte, dokumentiert, »namhaft«115 machte. Die Fotografie diente Charcot als Wissensspeicher, aber sie ist mehr als das: Sie symbolisiert die Macht des Sehens, das Blickregime der Salpetrière, wo sich die Körper der kranken Frauen unter dem Blick des Arztes als Darstellungen ihrer Leiden fügten, verbogen, affektierte, unmögliche Haltungen einnahmen, entrückten und kollabierten. Georges Didi-Huberman hat anschaulich gemacht, wie das symbiotische Verhältnis von Ärzten und Patientinnen, das auf einem System der Beobachtung, der Gesten und Weisungen einerseits, auf der Erwiderung, Selbstdarstellung und Bestätigung andererseits beruhte, als Grundlage eines neurotischen Schauspiels zu werten ist, das die Krankheit 〉Hysterie allererst zu konstruieren half. Wo Charcot mit der Fotografie als Medium der Pose arbeitete, nutzt Negro die neuen Möglichkeiten der Kinematografie. Der Ablauf der Szene gleicht jedoch einer öffentlichen Sitzung Charcots, der regelmäßig Vorlesungen abhielt und dabei Patientinnen ihre Symptome vorführen ließ. Eine ähnliche Vereinbarung scheint Negro mit der unbekannten Dame zu haben, deren Anfall zeitgerecht stattfindet, um Behandlung und Ergebnis auf eine Filmrolle zu bekommen.

Sigmund Freud hat 1885 während einer Studienreise Charcot und seine Behandlungsmethoden am Hôpital Salpetrière kennengelernt und auf dessen Einsatz der Hypnose seine Psychoanalyse aufgebaut. Freuds Rückführung der Leiden auf eine >Urszene<, die als reales Ereignis (oder tradierte Störung) den benennbaren Ursprung der Symptome darstellt, ist weniger auf eine bildliche denn sprachliche Aufarbeitung der Krankheit aus. »Die hysterischen Symptome sind Abkömmlinge unbewußt wirkender Erinnerungen ${ }^{116}$ die

\footnotetext{
115 Georges Didi-Huberman, Erfindung der Hysterie. Die photographische Klinik von JeanMartin Charcot, München 1997, S. 28.

116 Sigmund Freud, Gesammelte Schriften, Bd. 1: Studien über Hysterie, Frühe Arbeiten zur Neurosenlehre (1829-1899), Leipzig/Wien/Zürich 1925, S. 427.
} 
zwar sichtbar, aber nicht sagbar waren. Seine Methode war denn auch nicht das Schauspiel, sondern die analytische Sitzung, in der mittels des therapeutischen Gesprächs die Ursachen der Traumata ans Licht gebracht werden sollten. Freud hat sich auch über die Methoden seiner Kollegen geäußert, welche die Kriegsneurotiker >konservativ< behandelten. In seinem »Gutachten über die elektrische Behandlung der Kriegsneurotiker « von 1920 beschreibt er die Bewegungsstörungen, das Zittern und die Lähmungen, die er bei deutschen Soldaten beobachten konnte, als rein psychischer Natur, eine Flucht in die Krankheit aus Gründen der »Angst um das eigene Leben, Sträuben gegen den Auftrag andere zu töten, Auflehnung gegen die rücksichtslose Unterdrückung der eigenen Persönlichkeit durch die Vorgesetzten «. ${ }^{117}$ Die Einsicht, dass die Störungen nicht organischer oder funktioneller Natur seien, habe sich zwar unter den deutschen Militärärzten durchgesetzt, aber die behandelnden Ärzte stünden vor einem Dilemma: Die Wiederherstellung der »Kriegstüchtigkeit« als oberstes Ziel vor der »Herstellung des Kranken« werde als starke Belastung empfunden, stehe die Medizin damit doch nicht mehr im Dienst der Humanität, sondern des Volkskrieges. ${ }^{118}$ Freud lehnte es ab, dass die Kranken trotz dieser Erkenntnis als Simulanten behandelt und mittels schmerzhafter Starkstromtherapie so gequält wurden, dass ihnen, die vor dem Krieg in die Krankheit geflüchtet waren, die Rückkehr in den Kriegsdienst oft als bessere Alternative erschien. Die Traumatisierten seien so auch nie wirklich geheilt worden und wiesen, wieder im Feld, eine hohe Rückfallquote auf.

Zwei Dinge fallen an der Bearbeitung des historischen Materials durch Gordon auf: Einerseits greift er durch die Verlangsamung und Wiederholung im Loop in die zeitliche Struktur der Filme ein, andererseits verändert er ihre räumliche Wahrnehmung durch die Großprojektion auf freistehende Leinwände im Ausstellungsraum. Mit der Transformation auf Video wird die Zeitlupe allererst ermöglicht, und die Veränderungen durch sie sind radikal: War das Material zuvor bereits durch die technischen Gegebenheiten des Filmes ruckelig und zittrig und erforderte die Technik des gleichmäßigen Kurbelns viel Übung, um ungewollte Bildbewegungen zu vermeiden, ${ }^{119}$ so wird durch die zeitliche Streckung der Bilder eine bewusste Transformation in einen veränderten Bildrhythmus vorgenommen. Die Bewegungen des Kriegsneurotikers in $10 \mathrm{~ms}^{-1}$ wirken nun noch ataktischer, als sie bereits sind. Die quälenden Versuche des

117 Sigmund Freud, »Gutachten über die elektrische Behandlung der Kriegsneurotiker«, Niederschrift: 23.2.1920, in: Psyche 26, 1972, 942-945, S. 943.

118 Ebd., S. 944.

119 Vgl. hierzu die Hinweise von Martin Weiser (Medizinische Kinematographie, Dresden 1919) zur richtigen Handhabung des kinematografischen Apparats bei der Erstellung von Lehrfilmen. 
Aufstehens und das wiederholte Scheitern am eigenen Körper (der unversehrt, ja sogar muskulös und gesund wirkt, jedoch durch die psychische Kraft des Traumas in seine unnatürliche Haltung gezwungen wird) machen sichtbar, wie sich die psychische Störung körperlich äußert, und damit schließt Gordons Arbeit an den Diskurs der Sichtbarmachung des Unbewussten in den technischen Medien an, auf dem unter anderem Walter Benjamin seine »Kleine Geschichte der Photographie «(1931) ${ }^{120}$ aufgebaut hatte. Gordon knüpft an die ursprüngliche Rolle der Filme an, die im wissenschaftlichen Diskurs der Evidenzproduktion dienten: Mittels der filmischen Aufnahmen sollten, wie oben beschrieben, Krankheiten diagnostiziert und Simulanten entlarvt werden; es wurden, wie bei Hysterical, Standards der Anamnese in der Aufzeichnung und damit in der endlos zu wiederholenden Sichtbarkeit verfestigt. Ute Holl beschreibt, wie die »Evidenz des Filmischen «121 die Bewegungen der hysterischen Patientin zugleich hervorruft und aufzeichnet, so dass Subjekt und Objekt der Neuropathologie wie auch des Films ununterscheidbar werden: »Die Bewegung der Neuropatologia entsteht als Agencement, als Konstellierung von Visualisierungstechnik, Körper und Blicken im Bild. Die Evidenz einer Krankheit, die im Film illustriert und kanonisiert werden soll, erscheint somit sichtbar als Effekt des Dispositivs, das sie nur sichtbar machen sollte. « ${ }^{122}$ Das gleiche Zusammenspiel von filmischer Aufzeichnung, Darstellung durch die Patient/innen und Bedeutung des Materials innerhalb des medizinischen Diskurses kennzeichnet die anderen beiden beschriebenen Filme, wenn sie auch unter veränderten Vorzeichen entstanden sind. Gordon entzieht den wissenschaftlichen Film nun durch die Verschiebung in den Ausstellungsraum seinem Kontext der Evidenzproduktion: Die Besucher/innen als Adressaten begegnen dem Found Footage über die affektive Wirkung, welche die Bilder in ihrer dokumentarischen Funktion »ohne Code«(Barthes) und auf die Sichtbarkeit des Traumas zurückgeworfen auf sie haben. Bei Hysterical wie auch bei $10 \mathrm{~ms}^{-1}$ ist dies in erster Linie die entwürdigende Zurschaustellung der Patient/innen, die auf ihre Symptome und deren Heilung reduziert werden. Gordons zeitliche Bearbeitung - die Zeitlupe und die Wiederholung im Loop - nimmt dabei die den Symptomen zugrunde liegende Zeitstruktur des Traumas auf. Er entzieht das Material seiner narrativen Struktur von Diagnose, Anamnese und Heilung und somit der Lösung des medizinischen Problems. Indem er diese verweigert und den Zuschauer/innen die reine Sichtbarkeit

120 Walter Benjamin, »Kleine Geschichte der Photographie« (1931), in: ders., Das Kunstwerk im Zeitalter seiner technischen Reproduzierbarkeit, S. 45-64.

121 Holl, »Neuropathologie als filmische Inszenierung«, S. 223.

122 Ebd., S. 237. 
der Erinnerung in ihrer körperlichen Figuration präsentiert, verschafft Gordon seinen Werken durchaus eine ethische Dimension, da er die tatsächliche psychologische Verwundung der Kranken mittels der Zeitlupe und Wiederholung der Sichtbarkeit zuführt.

Emmanuel Alloa hat die Rolle des (Körper-)Gedächtnisses für das Trauma anschaulich beschrieben:

\begin{abstract}
Traumatische Ereignisse sind Ereignisse, die sich in einen Körper unwiderruflich einschreiben; im episodischen Gedächtnis jedoch lässt sich für sie kein Eintrag finden. Gerade deshalb, weil sie im Zeitstrahl des Erlebten keinen Platz finden, sind Traumata Widerfahrnisse, die nicht zu Erfahrnissen werden können: Als gelebtes Ereignis, das deshalb, weil es die Ökonomie des psychischen Apparats sprengt, zu keinem Erlebnis werden kann, ist das Trauma grundsätzlich a-topisch; als ein Dass, das sich in kein Wann und Wo normalisiert, wirkt es in der Nachträglichkeit einer Heimsuchung fort. ${ }^{123}$
\end{abstract}

Der Begriff der Nachträglichkeit betrifft bei Freud seine Konzeption der Zeitlichkeit und der psychischen Kausalität. ${ }^{124}$ Er ist nicht auf die allgemeine Wirkung der vergangenen Erlebnisse auf die Gegenwart zu reduzieren, sondern bezeichnet die nachträgliche Umarbeitung oder Umordnung von Erlebtem, das wie ein traumatisches Ereignis im Augenblick des Erlebens nicht vollständig begriffen werden konnte. Verdrängte Erinnerungen werden durch die Aufarbeitung also nachträglich zum Trauma, sie erhalten »gleichzeitig einen neuen Sinn und eine neue psychische Wirksamkeit«.125 Das medizinische Symptom als körperliche Sichtbarmachung eines unbewussten Traumas hat dabei eine Zeitstruktur, die in beide Richtungen, die Vergangenheit und die Zukunft, wirkt - Nachträglichkeit bedeutet eine Durchdringung von Vergangenheit, Gegenwart und Zukunft, die nicht linear in eine Richtung und damit kausal und irreversibel als Abfolge von Ereignissen und Zuständen erfolgt, sondern wechselseitig. Das psychoanalytische Konzept der Nachträglichkeit des Symptoms weist, wie Georges Didi-Huberman gezeigt hat, Parallelen zum Nachleben der Bilder in der Pathosformel bei Aby Warburg auf:

Das Freudsche Modell des Symptoms erlaubt es uns in der Tat, die Plastizität der Verkörperung und die Zeitlichkeit des Nachlebens - in ein und derselben Pathosformel - zu vereinen. Die Symptombildung ist in gewisser Weise

123 Emmanuel Alloa, »Eingefleischte Gesten. Nachleben und visuelle Zeugenschaft in Claude Lanzmanns Shoah und Rithy Pans $S_{21 \ll,}$ in: Peter Geimer, Michael Hagner (Hg.), Nachleben und Rekonstruktion. Vergangenheit im Bild, München 2012, S. 206-229, S. 206 f.

124 Vgl. Jean Laplanche, Jean-Bertrand Pontalis, Das Vokabular der Psychoanalyse, Erster Band, Frankfurt am Main 1973, S. 313-317 (Eintrag »Nachräglichkeit, nachträglich«).

125 Ebd., S. 313 . 
ein Nachleben, das seine Verkörperung erfährt. Körper, die von Konflikten, von widersprüchlichen Bewegungen und den Strudeln der Zeit heimgesucht werden. Körper, aus denen plötzlich ein verdrängtes Bild hervortritt, wie Warburg erkannte, als er die Zählebigkeit, das Auftauchen und den Anachronismus des Nachlebens vor dem Hintergrund des Vergessens, der Latenzen und Verdrängungen beobachtete. Es ist erstaunlich, daß Freud im Symptom eine in allen Punkten ähnliche Zeitstruktur entdeckte. ${ }^{126}$

Das »diskontinuierliche Zeitregime $\ll^{127}$ der Wiederholung verbindet den nicht konkret historisch zu fixierenden Ursprung des Traumas, das in der körperlichen Symptomatik in der Gegenwart figuriert, mit der Logik des Nachlebens von Bildern in Pathosformeln, wie sie oben beispielsweise im Zusammenhang mit der Pose beschrieben wurden. Gordons erweiterte Sichtbarmachung überträgt dies auf die zeitliche Bearbeitung der Filme: »Die Pathosformel der Wiederholung, ihre gesteigerte Version, ist die Zeitlupe.«128 Eine finale Wiederholung dieser Verweisstruktur findet man, wenn man in Betracht zieht, dass Gordon sich innerhalb des Diskurses des Found-Footage-Films bewegt. Für diesen spielt die Wiederholung von verdrängten Bildern per se eine wichtige Rolle, denn häufig wird in den Archivkunstfilmen der 196oer und 1970er Jahre (zum Beispiel bei Bruce Conner), und den späteren Essayfilmen (Chris Marker) auf politische und soziale Themen, auf Fernseh- und Wochenschaubilder zurückgegriffen. Der Bezug zur Psychoanalyse sei unübersehbar, stellt Sharon Sandusky fest:

Wie jene verdrängten Erfahrungen der frühesten Kindheit, die man überhaupt nur bemerkt, wenn man das Trauma noch einmal durchlebt, wird auch die sozialkritische Interpretation des historischen Ereignisses und seiner psychischen Konsequenzen möglich, indem man sich dem Filmartefakt bewußt aussetzt. Die Aufgabe des Archivkunstfilms ist nicht viel weniger als die einer kulturellen Psychotherapie, statt der >talking cure< eine >filmic cure< sozusagen. ${ }^{129}$

Interessanterweise taucht ein Aspekt der männlichen Hysterie auch bildthematisch im Found-Footage-Film auf, nämlich die Visualisierung der schockhaften Verunsicherung der Moderne, welche an der Wende zum 2o. Jahrhundert gleichermaßen dem Film wie der Eisenbahn zugeschrieben wurde. Beide Dispositive bewirkten innerhalb kürzester Zeit eine Verdichtung

\footnotetext{
126 Didi-Huberman, Das Nachleben der Bilder, S. 341.

127 Ebd., S. 35o.

128 Andreas Cremonini, Valentin Groebner, »Es kommen sehen. Johan Grimonprez' Video Dial H-I-S-T-O-R-Y«, in: Geimer, Hagner, Nachleben und Rekonstruktion, S. 134-157, S. 15 o.

129 Sharon Sandusky, »Archäologie der Erlösung. Eine Einführung in den Archivkunstfilm«, in: BLIMP - Zeitschrift für Film 16, 1991, S. 14-23, S. 16.
} 
von Zeit und Raum, was die zeitgenössischen Betrachter/innen beziehungsweise Fahrgäste häufig überforderte. ${ }^{130}$ Die Symptomatik von Hysterie und Trauma, wie sie Freud und andere Psychoanalytiker des 19. und frühen 20. Jahrhunderts beschrieben haben (Charcot, Breuer, Binswanger), hat ihre Wurzeln in solchen Veränderungen durch die Modernisierung und Industrialisierung. Die Symptome weiblicher Hysterie werden im Kern auf die rigiden patriarchalen Strukturen der bürgerlichen Familie zurückgeführt, diejenigen der männlichen Hysterie und Traumatisierung vor allem auf technischen Fortschritt und seine Wirkung auf die Wahrnehmung, ausgelöst durch die mit der Eisenbahn einhergehenden traumatischen Erfahrungen von Eisenbahnunglücken und schließlich die militärischen Erfahrungen im Ersten Weltkrieg. ${ }^{131}$ Entsprechend wurde die männliche Hysterie zuerst von Charcot, später auch von anderen Nervenärzten, am Beispiel der Opfer von Eisenbahnunglücken beschrieben, bevor die Menge der Soldaten mit posttraumatischem Belastungssyndrom, wie man heute diagnostizieren würde, den Begriff des Kriegsneurotikers prägte.

\section{Fazit}

Douglas Gordons zeitgenössische Wiederaufnahme der wissenschaftlichen Filme schließt an die Art und Weise der archäologischen Herangehensweise an, wie sie auch dem Found-Footage-Film der 196oer Jahre eigen ist: Er verwendet Archivmaterial, das einem spezifischen, in diesem Fall medizinischen, aber durch die militärische Relevanz letztlich auch gesellschaftspolitischen Kontext zugehörig ist. Im Gegensatz zu den Filmemacher/innen der 196oer Jahre, welche die Mittel der filmischen und räumlichen Montage nutzten, die in der Kollision von Bildern die zugrunde liegenden Bedeutungsschichten freilegen kann, arbeitet Gordon vor allem mit zeitlichen Mitteln wie der videografischen Verlangsamung, der Doppelprojektion und der veränderten räumlichen Anordnung im Ausstellungsraum. Seine Projektionen entsprechen nicht dem kinematografischen Dispositiv der vor einer Leinwand fixierten Betrachter/innen, vielmehr können die freistehenden Leinwände von allen Seiten gesehen und angefasst werden, sie erzeugen eine ungewohnte Nähe und erregen durch die bewegten Bilder eine auch körperliche Affizierung. Die hier besprochenen Werke fügen sich in Gordons CEuvre insofern ein, als er die Arbeit mit gefundenem Material auch auf Hollywoodfilme angewandt hat;

130 Vgl. zur Eisenbahn im Found-Footage-Film das Kapitel 2 (»Material und Umformung, Reprise: der Lumièrsche Zug in der Avantgarde«) in Blümlinger, Kino aus zweiter Hand.

131 Vgl. zur kulturhistorischen Bedeutung des Traumas u. a. E. Ann Kaplan, Trauma Culture. The Politics of Terror and Loss in Media and Literature, New Brunswick, NJ 2005. 
die Möglichkeit, am heimischen Videorekorder endlos vor- und zurückzuspulen und so ikonische Filmszenen immer und immer wieder zu betrachten, hat Gordon selbst als eine Quelle seiner Arbeitsweise beschrieben. Die Thematik der Psychopathologie ist dominierend in seinen Werken dieser Jahre und auf sein Interesse am »Dauerthema der gespaltenen Persönlichkeit« zurückzuführen, das die Psyche ins Zentrum seiner Ästhetik rückt, wie Lynne Cooke schon 1998 festgestellt hat: »Dieses Interesse schließt Extreme geistiger und mentaler Verfassung ein, Zustände von Psychose und Ekstase, von Wahnsinn und Euphorie, und es ist, wenn auch nicht eindeutig, im Tiefsten moralisch, um nicht zu sagen religiös. «132

Die hier besprochenen Arbeiten gestalten sich jedoch komplexer, wie die Analyse zeigen sollte, da sie auch auf die Visualisierungsstrategien des frühen wissenschaftlichen Films Bezug nehmen und die Wiederholung als zentrales ästhetisches Prinzip aufzeigen, das als Zeitstruktur alle drei angesprochenen Bedeutungsebenen durchdringt: die der kinematografischen Installation (Loop), des hysterischen Traumas (Latenz) und des Found Footages (Wiederaufnahme archivarischen Filmmaterials). Die Arbeiten thematisieren zwei psychoanalytische Topoi, einerseits die Rolle des Unbewussten in der Hysterie, zum anderen die dem Trauma und der Hysterie inhärente Zeitstruktur der Wiederholung. Lynne Cooke führt Gordons Faszination für die medizinischen Filme (und somit auch die Faszination der Betrachtenden) auf den Voyeurismus und Sadismus zurück, der zutage tritt, »wenn Filmmaterial dieser Art die pädagogische Bedeutung genommen und es als Schauspiel ausgebeutet wird «. ${ }^{133} \mathrm{Im}$ Gegensatz zu dieser Lesart, welche die psychopathologische Störung letztlich im Blick der Betrachtenden verortet, sollte hier deutlich gemacht werden, wie sich auf der Ebene des bewegten Bildes die körperliche Figuration des Traumas Bahn bricht und die mediale mit der motorischen Dysfunktion einhergeht. Die Geschichtlichkeit des Materials ist in der Bearbeitung durch Gordon untrennbar mit der Zeitlichkeit der Wahrnehmung der bewegten Bilder verwoben.

132 Lynne Cooke, »Douglas Gordon. `Ein visueller Mensch, einer, der sehen kann««, in: Eckhard Schneider (Hg.), Douglas Gordon, Ausstellungskatalog Kunstverein Hannover, Hannover 1998, nicht paginiert. Cooke bezieht sich hier auf die Spielfilmadaptionen 24 Hour Psycho, Confessions of a Justified Sinner und Between Darkness and Light (After William Blake), in denen Gordon bewusst Filme verwendet, die psychische Extreme thematisieren - die Psychose in Psycho, 1960, USA (Alfred Hitchcock), die religiöse Entrückung bzw. Besessenheit in Das Lied von Bernadette, 1943, USA (Henry King), und Der Exorzist, 1973, USA (William Friedkin), sowie die Schizophrenie in Dr. Jekyll und Mr. Hyde, 1931, USA (Rouben Mamoulian).

133 Lynne Cooke, »Douglas Gordon«, nicht paginiert. 


\section{Erinnerungsbilder und Diskursanalyse}

Die Phoenix Tapes des Künstlerduos Matthias Müller und Christoph Girardet von 1999 sind gleichzeitig Film und Installation: Es handelt sich um sechs katalogartige Bilderserien, die entweder als simultane 6-Kanal-VideoInstallation auf Bildschirmen oder einzeln hintereinander gezeigt werden, jeweils zwischen vier und zwölf Minuten lang. Das Video Bedroom bezieht sich auf die typischen Orte der Frauen im Hollywoodfilm: Hier winden sie sich im Schlaf, stehen wartend am Fenster, sitzen weinend im Sessel; hier werden sie von Männern umarmt und stehen gelassen; hier wird mithilfe von Puder, Schmuck und eleganter Kleidung Haltung bewahrt, während das Gesicht im Close-up immer wieder Verzweiflung überfliegt; hier wird aus der männlichen Umarmung schließlich ein Ringen und Kampf bis zum Tod (Abb. 13).

Müller und Girardet, für deren umfassende gemeinsame Arbeit die Phoenix Tapes stellvertretend stehen, verwenden in den sechs Filmen ausschließlich Ausschnitte mit Leitmotiven aus Filmen von Alfred Hitchcock - neben den in Bedroom gezeigten Frauenbildern beispielsweise auch eine Kompilation von Close-ups auf bedeutungstragenden Gegenständen, bei Hitchcock >McGuffins< genannt, Schlüssel, Koffer und Schilder, oder die Dynamiken innerhalb des Verhältnisses der Mütter zu ihren Söhnen.

Matthias Müllers Arbeit als Filmemacher dreht sich um das Bedürfnis, »das Eigene im Fremden zu erkennen, gleichzeitig Fremdes im vermeintlich Eigenen aufzuspüren ${ }^{134}$ Er arbeitet seit 1985 mit einem Archiv aus Filmen, die er per Video aus dem Fernseher aufgenommen hat. Mit Christoph Girardet, mit dem er zusammen an der Kunsthochschule Braunschweig bei der Experimentalfilmerin Birgit Hein studierte, verbindet ihn die »Leidenschaft für das Sammeln und Neuordnen von Erinnerungsstücken « ${ }^{135}$ Die individuellen Sammlungen entspringen dem heimischen close reading am Fernseher, jedoch, wie Müller behauptet, ohne »Formel«, sondern auf der Grundlage von vager, obskurer Faszination. ${ }^{136}$ Die Auswahl ist dabei scheinbar kontingent: »Ich sammele

134 Kathrin Becker, »Beyond and Back. Über das Fremde und das Eigene in den Arbeiten von Matthias Müller«, in: Matthias Müller, Album. Film, Video, Photography, Ausstellungskatalog Neuer Berliner Kunstverein, Berlin 2004, Frankfurt am Main 2004, S. 27-33, S. 27 f.

135 Stefanie Schulte Strathaus, »ALL THAT HEAVEN ALLOWS. Atem. Wege. Zwischen Kino und Kunst«, in: dies. (Hg.), The Memo Book. Filme, Videos und Installationen von Matthias Müller, Berlin 2005, S. 6-41.

${ }_{136}$ Müller in einem Interview mit Alena Williams: »There definitely is no formula for the selection and collection of particular motifs. Fascination is crucial, and it may be a vague, or even quite obscure, one.« (Alena Williams, »Interview with Matthias Müller«, in: dies. [Hg.], Light is a kind of rhythm, Stuttgart 2009, S. 17-19, S. 17.) 

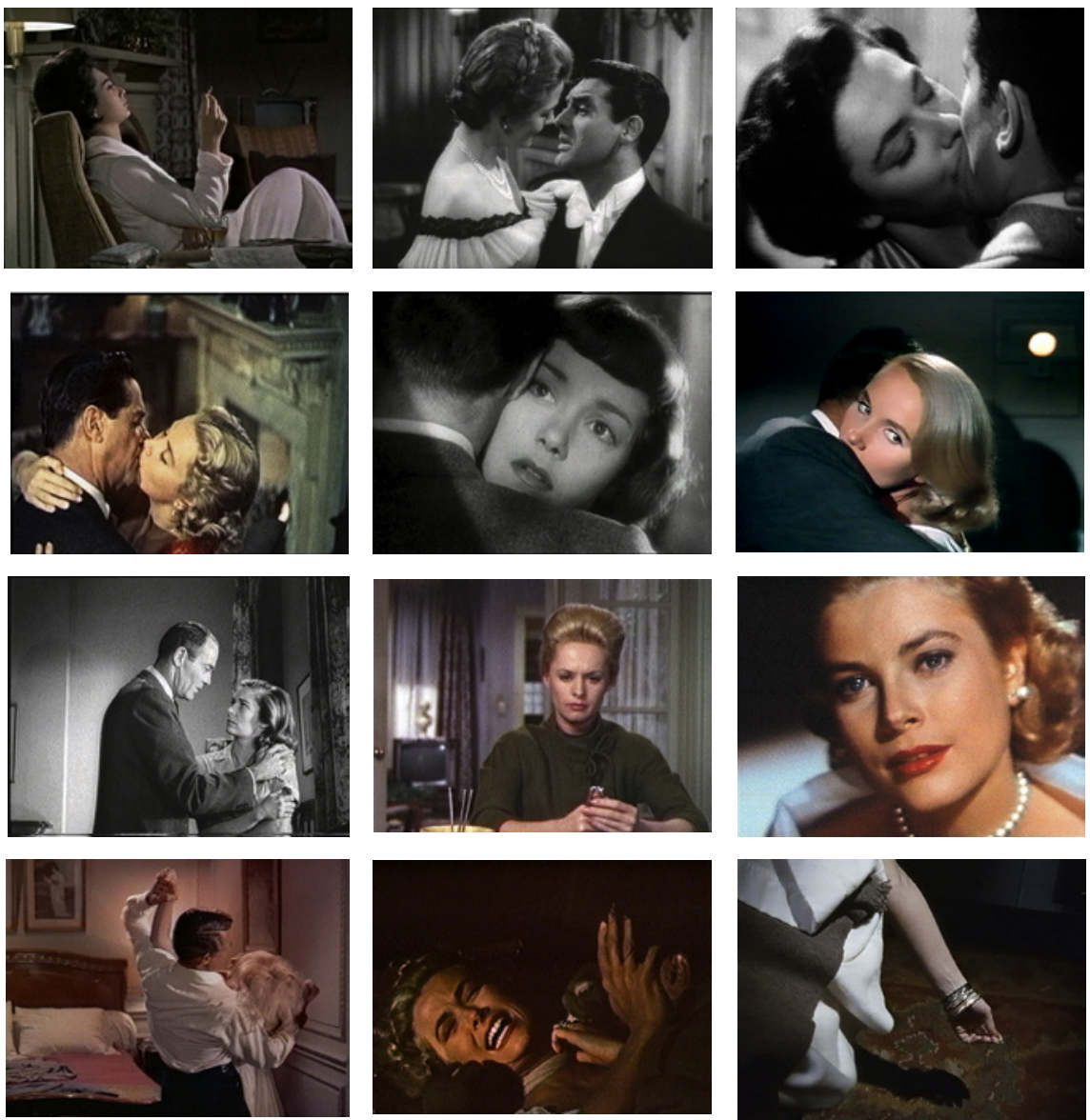

Abb. 13 Videostills, aus: Christoph Girardet / Matthias Müller, Phonix Tapes (Bedroom), 1999

Strandgut in meinem Wohnzimmer, und zwar mit der TV-Fernbedienung. «137 In ihrer generationstypischen Fernsehsozialisation ähneln Christoph Girardet und Matthias Müller Douglas Gordon, der seine Vorliebe für Noir-Filme ebenfalls auf nächtliche Sessions mit Fernseher und Videorekorder zurückführt. Im Gegensatz zu Gordon grenzt sich Müller jedoch dezidiert von einem Vorgehen ab, das aus Zergliederung und Analyse oder aus der gezielten Zerstörung der Bilder besteht: Die Kreation eines neuen Werks aus den am Leben gehaltenen

137 Mark Gisbourne, »Leben mit Stachelschweinen«, in: Matthias Müller, Album. Film, Video, Photography, Ausstellungskatalog Neuer Berliner Kunstverein, Berlin 2004, Frankfurt am Main 2004, S. 35-67, S. 45 . 
Versatzstücken steht für ihn als Filmemacher im Mittelpunkt. Dieses Werk beruht im Kern auf der Montage sich ähnelnder Fragmente, das heißt auf der zeitlichen innerfilmischen Neustrukturierung, die sich gegebenenfalls in die Installation auffächert, und nicht auf der (kunst-)räumlichen Entfaltung des Materials, die auf dem Wechsel des Dispositivs beruht.

Die in Bedroom collagierten Szenen folgen einem homologen Ordnungssystem. Vier Prinzipien sind dabei augenfällig: zum einen bei der Auswahl der Szenen die Ähnlichkeit der Gesten, Blicke und Handlungen und die Regelmäßigkeit, mit der diese Motive innerhalb der vielen verschiedenen Filme auftauchen; zum anderen bei der Anordnung innerhalb von Bedroom die Serienbildung und Gruppierung. Die einzelnen Abschnitte deklinieren bildliche Motive am weiblichen Körper durch, jedoch nicht über ikonografische Bezüge, sondern über Blickkonstellationen und -intensitäten, Stimmungen und einzelne Gesten. Sie sammeln Wiederholungen, die Motive in Kadrierung und Dauer leicht variieren und dadurch zeigen, mit welchem Vokabular Hitchcock seine Frauenbilder erstellte.

Müller und Girardet gehen in ihren Mikroanalysen nach einem Verfahren vor, das dem diskursanalytischen historischen Verfahren, wie es Michel Foucault beschreibt, gleicht: Geschichtsschreibung muss sich demnach von der Idee von Kontinuität, von Traditionen und kausalen Entwicklungen abwenden und das Dokument anders bewerten, "sie sucht nach der Bestimmung von Einheiten, Mengen, Serien, Beziehungen in dem dokumentarischen Gewebe selbst «. ${ }^{138}$ Ausgehend von einer diskontinuierlichen Geschichte wird in Serialitäten von Bildern oder Objekten versucht, Gemeinsamkeiten zu entdecken: Es gilt, wie Foucault sagt, »die verschiedenen, verschränkten, oft divergierenden, aber nicht autonomen Serien zu erstellen, die den >Ort< des Ereignisses, den Spielraum seiner Zufälligkeit, die Bedingungen seines Auftretens umschreiben lassen «.139 Das dokumentarische Gewebe ist hier das 〉Material « der Filme, mit denen sie arbeiten - diese verweisen auf verschiedenste Diskurse: das System Hollywoodkino mit Studio-Mise-en-scène und Bildästhetik, die Bildsprache Hitchcocks, den Frauentypus und sein ambivalentes, zwischen Verführung, Unterdrückung und Gefahr changierendes Verhältnis zum Mann, das Gestenrepertoire und vieles mehr. Müller und Girardet begeben sich in einem vergleichenden Verfahren auf die Suche nach Bildern, die über ihre Bedeutung innerhalb der Erzählung hinaus eine neue Regelmäßigkeit dieser genrespezifischen Codes aufweisen.

138 Foucault, Archäologie des Wissens, S. 14.

139 Michel Foucault, Die Ordnung des Diskurses, Frankfurt am Main 1991, S. 36. 
Bezeichnend ist dabei, wie sich in der Kombination gleichartiger Szenen trotzdem eine Dramaturgie herauskristallisiert: Die Überleitung von einer Szene in die nächste folgt einer fast natürlich anmutenden Migration von Formen im Modus ihrer Variation und Wiederholung. So wird die ikonografische Ähnlichkeit der Hitchcock'schen Umarmung zum tödlichen Würgegriff nicht einfach festgestellt, sondern die Ambivalenz beider Motive, ihre strukturelle Untrennbarkeit, offengelegt. Die sich wie zwangsläufig ergebende lineare Lesbarkeit scheint im Medium des Spielfilms bereits angelegt zu sein. Erneut mit Foucault argumentierend, könnte man dies darauf zurückführen, dass es gar nicht möglich ist, außerhalb des machtpolitischen Ordnungsmodells des strukturierenden Diskurses Sinn zu generieren: Es ist hier das Ordnungsmodell des narrativen Films, das die Szenen bereits in ihrem inneren Aufbau so vorstrukturiert, dass sich Linearität und Narration von selbst einzustellen scheinen. Zugleich wird diese Linearität in der Analyse durch die Betrachterin oder den Betrachter reproduziert. Jedoch auch aus kunst- beziehungsweise filmhistorischer Perspektive muss das Erzählkino als zentraler Bezugspunkt gewürdigt werden, in negativer wie in positiver Hinsicht - negativ, weil es mit dominanten Erzählstrukturen die flexible Bildlogik unterdrückt, positiv, weil es mit starken Bildern aufwarten kann.

Die filmischen Bilder werden von Müller/Girardet stärker als beispielsweise bei Gordon oder Coleman auf ihr imaginatives Potenzial hin geöffnet: Die Neukontextualisierung zielt nicht auf eine die Distanz wahrende Betrachtung oder auf eine immersive Wiederbelebung, sondern auf eine Fortsetzung der Geschichte mit neuen Mitteln. Müller und Girardet geht es nicht um eine Destruktion des Erzählkinos, sondern gerade um die Wiedererkennung des eigenen Bilderdenkens im Formenrepertoire des anderen Autors. Die angesprochene suggerierte Kontingenz ist dabei trügerisch, da Müller selbst den kalkulierenden Charakter seiner Arbeit betont und sich von einer experimentellen Arbeitsweise distanziert. ${ }^{140}$ Der personalisierte Zugang, der schließlich im Werk von Müller die Unterscheidung von selbst gedrehtem und angeeignetem Material hinfällig macht, ist bereits in dem selbst geschaffenen Archiv, mit dem gearbeitet wird, angelegt. Das Footage fungiert somit als Spiegel des archäologisch arbeitenden Künstlers - zu Recht hat Christa Blümlinger den Kollektionismus Müllers mit einem Selbstporträt gleichgesetzt. ${ }^{141}$

140 Vgl. Williams, »Interview with Matthias Müller«, S. 18.

141 Blümlinger, Kino aus zweiter Hand, S. 107. 


\section{Possessive Spectator}

Müller und Girardet widmen sich in ihren Arbeiten überwiegend dem narrativen Hollywoodfilm und spielen somit auch auf einen bestimmten Zuschauertypus an, der in der Ästhetik und Dramaturgie dieser Filme vorausgesetzt wird. Laura Mulvey hat ihn als possessive spectator beschrieben, in Unterscheidung zum pensive spectator. ${ }^{142}$ Der possessive spectator verkörpert den/die Zuschauer/in, der/die vom Star eines Films wie magisch angezogen ist und in einem fetischisierten Verhältnis zu dessen Bild, seinem Körper und seiner Art der Darstellung steht. Dieses Verhältnis wurde besonders im Starkult der 1920er bis 196oer Jahre gepflegt, als Diven wie Marlene Dietrich oder männliche Stars wie Robert Mitchum zur tragenden Figur eines Films stilisiert wurden. In einer standardisierenden Filmindustrie, die auf der Faszination einzelner Personen basiert, deren Rollenverhalten durch alle anderen Aspekte der Dramaturgie, Mise en scène und Montage unterstützt wird, spielt die Wiedererkennbarkeit eine wichtige Rolle, sie ist der Garant für den Erfolg des Films, wie Mulvey ausführt: When a film industry streamlines its star system, instantly recognizable, iconic screen actors produce a highly stylized performance, enhanced by an highly stylized star-focused cinema.«143 Das Spiel des Stars ist in der Regel der Motor der Narration und Bewegung im Film, doch zugleich muss er das Gegenteil beherrschen: »But the great achievement of star performance is an ability to maintain a fundamental contradiction in balance: the fusion of energy with a stillness of display, However energetic a star's movement might seem to be, behind it lies an intensely controlled stillness and an ability to pose for the camera. ${ }^{144}$ Das erfolgreiche Schauspiel der Stars beruht auf ihrer Fähigkeit, Bewegung und Pose miteinander zu verbinden, und genau an diesen Momenten der Verlangsamung und teilweise Stillstellung filmischer Narration, die sich bereits in den Filmen selbst feststellen lassen, setzt die Intervention von Müller/Girardet, aber auch von Douglas Gordon in 24 Hour Psycho an. Denn durch die technischen Möglichkeiten des privaten Videokonsums, dem heute die Clipkultur von Youtube, Twitter und anderen Plattformen an Computer, Tablet oder Smartphone entspricht, wird dem zwanghaften, fetischisierten Verlangen nach dem Bild der Stars, das vormals über Fotos, Autogrammkarten und Poster des Objekts der Begierde habhaft wurde, auch im bewegten Bild Rechnung getragen:

\footnotetext{
142 Vgl. Mulvey, Death $24 x$ a Second.

143 Laura Mulvey, »The Possessive Spectator«, in: dies., Death $24 x$ a Second, S. 161-18o, S. 162.

144 Ebd.
} 
With electronic or digital viewing, the nature of cinematic repetition compulsion changes. As the film is delayed and thus fragmented from linear narrative into favourite moments or scenes, the spectator is able to hold on to, to possess, the previously elusive image. In this delayed cinema the spectator finds a heightened relation to the human body, particularly that of the star [...] this new stillness exaggerates the star's iconic status. ${ }^{145}$

Wie neben Phoenix Tapes insbesondere die Arbeit Kristall (2006) zeigt, gelingt es Müller und Girardet durch ihre Found-Footage-Arbeiten, das innige Verhältnis des Kinopublikums zum Bild seines Stars, ob männlich oder weiblich, in prägnante Bilder zu fassen. Durch die zeitliche Bearbeitung zum Beispiel durch Zeitlupe und die wie besessene Wiederholung einzelner Szenen wird das Bild des Stars aus seiner narrativen Verankerung gelöst und sein Körper dem fetischisierenden Blick der Betrachtenden ausgesetzt. Bezeichnend für diese Art der Found-Footage-Bearbeitung sind auch die Werke des österreichischen Experimentalfilmers Martin Arnold, vor allem seine drei Filme der Cinemesis-Reihe Pièce Touchée (1989) (Abb. 14), Passage à l'Acte (1993) und Alone. Life Wastes Andy Hardy (1997), die er mittels eines Optical Printers am Filmschnittplatz herstellte. Sie wurden von William C. Wees als »the most extreme examples of the critical, analytical, and deconstructive tendencies in the avant-garde's appropriation of Hollywood images « ${ }^{146}$ bezeichnet. Pièce Touchée ist ein 16-minütiger Film, der aus den Filmkadern einer 18 Sekunden langen Szene von The Human Jungle aus dem Jahr 1954 besteht, einem wenig bekannten Kriminalfilm von Regisseur Joseph M. Newman mit Gary Merrill und Jan Sterling in den Hauptrollen. Darin sitzt ein Frau lesend im Sessel, als ihr Ehemann die Wohnung betritt. Er küsst sie, sie steht auf, und sie gehen gemeinsam aus dem Bild. Es ist eine unbedeutende Szene, an der man aber trotzdem einige filmische Darstellungscodes der 195oer Jahre (Bildaufbau, Studiobeleuchtung) und im Verhalten des Paares soziale Normen der Geschlechterdifferenz ablesen kann. ${ }^{147}$ Arnold seziert durch stroboskopartiges Hin- und Herschalten zwischen den Bildern jede einzelne Bewegung und verkehrt die Raumwirkung durch Spiegelung und Auf-den-Kopf-Stellen

\footnotetext{
145 Ebd., S. 161.

146 William Charles Wees, »The Ambiguous Aura of Hollywood Stars in Avant-Garde FoundFootage Films«, in: Cinema Journal 41/2, Winter 2002, S. 3-18, S. 15.

147 Martin Arnold in Scott McDonald, »Sp ... Sp ... Spaces of Inscription. Scott McDonald im Gespräch mit Martin Arnold «, in: Alexander Horwath, Lisl Ponger, Gottfried Schlemmer (Hg.), Avantgardefilm. Österreich. 1950 bis heute, Wien 1995, S. 285-300, S. 289. Siehe zu Pièce Touchée auch den Text von Maureen Turim »Eine Begegnung mit dem Bild« im selben Band.
} 

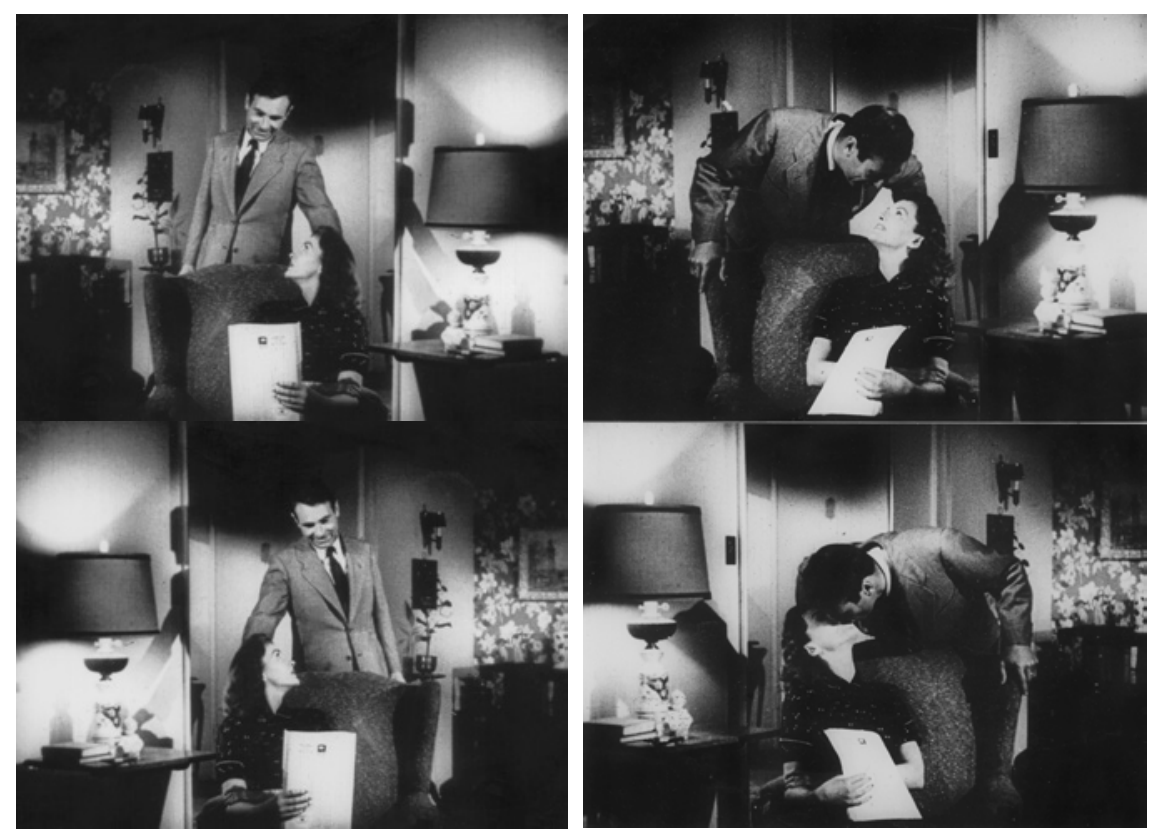

Abb. 14 Filmstills, aus: Martin Arnold, Pièce Touchée, 1989

der Bilder, so dass sie kollidieren oder in kreisenden Bewegungen herumwirbeln. Die Beobachtung einzelner gestörter Bewegungsabläufe im Bild, zum Beispiel ein Zucken des Fingers der Frau, verwandelt sich mit zunehmender Ausweitung der Störung auf die Raumordnung in eine Wahrnehmung von Bewegungen auf der Oberfläche des Bildes. Von einem >lesbaren< Bild, in dem eine konkrete Bewegung zu beobachten ist, wird es so zu einem abstrakten, von einem visuellen >Rauschen< erfüllten Bild. Arnold unterwirft die ganze Szene einer $\gg$ Art Takt, eine $[\mathrm{r}]$ metrisch non-humane[n] Bewegung [...]. Es ist eine seltsam unentschlossene, beständig verzögerte, in Minimalintervallen aufgelöste, nervöse Taktik zwischen vor und zurück, zwischen Tun und Nicht-Tun, zwischen Wollen und Nicht-Wollen.«148 Ähnlich verfährt Arnold mit Mickey Rooney und Fay Holden in einem Andy-Hardy-Film, ${ }^{149}$ wo die zärtliche Verabschiedung des Sohnes von der Mutter durch die beständige Wiederholung und Fragmentierung der Bewegung einen ödipalen Unterton erhält.

148 Marc Ries, Pièce Touchée (Synopsis)«, https://www.film.at/pi_ce_touch_e [26.4.2020].

149 Andy Hardy war der Name einer Rolle, die Mickey Rooney in einer erfolgreichen MGMFilmserie zwischen 1937 und 1947 spielte. 
Arnold selbst sagt zu seinem Interesse an den Spielfilmen: »Hollywoodfilme sind Filme die ausgrenzen, reduzieren, ablehnen: repressives Kino. Hinter dem, was dargestellt wird, versteckt sich immer etwas, das nicht gezeigt wird. Genau das macht es so interessant, sich damit zu beschäftigen.« ${ }^{150} \mathrm{In}$ seiner Aufdeckung unbewusster Bewegungen und versteckter Bedeutungen in Mustern und Codes, die die Narration des Hollywoodkinos bestimmen, ähnelt Arnolds Arbeitsweise 24 Hour Psycho von Douglas Gordon, wo die Drosselung des Metrums des Films unbewusste Bewegungen, nicht erinnerte Szenen und erst durch die Langsamkeit zu erkennende Details enthüllt. Während Gordon jedoch die Struktur des Films unangetastet lässt, greift Arnold in das Material ein, dupliziert es und unterlegt es mit einem kleinen Stück Tonschleife, aus dem ein hämmernder Sound wird. Es ist, so wird deutlich, vor allem die Manipulation der Verlaufszeit des filmischen Bildes, die in den Found-FootageArbeiten von Gordon, Müller/Girardet und Arnold die fiktionale Immersion und die Verwobenheit der Betrachtenden mit der kontinuierlichen Filmerzählung, der Filmzeit und dem Filmraum durchbrechen und dem einzelnen Frame oder >Sequenzbild< (Burgin) eine gewandelte Bedeutung zukommen lassen. Die bewegten Bilder tragen so ihre geschichtliche Gebundenheit an ein traditionelles Bild- und Erzählsystem nach außen. In der >Wiederverwertung< durch die Künstler mittels Verlangsamung, Montage oder Beschleunigung zeigt sich einerseits der modernistische Gestus der Reflexivität durch Dekonstruktion tradierter Sehgewohnheiten; andererseits entstehen jedoch Werke, deren visuelle und, zum Teil, auditive Kraft als eine Art »aggressiver Theatralität «151 das Publikum erneut fasziniert und an das Bildgeschehen bindet.

\section{"Das Schweigen des verlorenen Objekts«}

Im Gegensatz zu den vorausgegangenen Arbeiten stellt das Künstlerbuch FLOH (2001) von Tacita Dean keinen Bezug zu filmischen Archiven und deren zeitgenössischer Adaption mittels Video her. Es handelt sich im OEuvre der Künstlerin, die zu gleichen Teilen mit Film, Fotografie und Zeichnung arbeitet, um eine paradigmatische Arbeit, da sie die Sammelleidenschaft Deans zum Ausdruck bringt. ${ }^{152}$ FLOH besteht aus Fotografien, deren Herkunft, Alter und

\footnotetext{
15 O Martin Arnold, The Cineseizure, DVD Edition ARGE Index, Re:Voir, Medienwerkstatt Wien, Zitat auf DVD-Hülle.

151 Robin Curtis, Christiane Voss, »Editorial. Theorien ästhetischer Immersion«, in: montage $A V_{17} / 2,2008$, S. 4-10, S. 5 .

$15^{2}$ Sammlungen von Bildern, Postkarten und Objekten tauchen in Deans Werk immer wieder auf, beispielsweise Washington Cathedral (2002), bestehend aus 142 gefundenen Postkarten, ihre bis heute unabgeschlossene Sammlung von Kleeblättern, die Arbeit Die
} 
Urheber/innen unbekannt sind und die Dean in dem Buch und der dazugehörigen Sammlung von Abzügen ohne Kommentar kombiniert hat. Das Buch, im Steidl Verlag in einer nummerierten Auflage von 4000 Stück erschienen, gibt keine Hinweise auf Entstehungszusammenhang oder -datum, eventuelle Bearbeitung, Material oder Originalgröße der darin abgedruckten SchwarzWeiß- und Farbfotos. Dean verfährt hier ähnlich wie Douglas Gordon mit seinen filmischen Fundstücken: Die Fotografien werden den Betrachtenden beziehungsweise Lesenden völlig dekontextualisiert in veränderter Form angeboten, und es bleibt ihnen überlassen, aus dem Werk Rückschlüsse auf die Historizität der Stücke und auf die Intention der Künstlerin zu ziehen.

Was verraten uns die Bilder in der gegebenen Präsentationsform? Zunächst ist festzustellen, dass nur wenige Seiten mehr als ein Foto zeigen. Einzelne Fotografien sind stark vergrößert über zwei Seiten randlos abgedruckt, andere, offensichtlich zusammenhängende Fotos werden als Serie auf ausfaltbaren Seiten präsentiert, einige wenige Bilder sind alleine, nur einer weißen Seite gegenüber abgedruckt. Alle Bilder weisen einen wahrnehmbaren historischen Index auf, das heißt sie verweisen aufgrund medialer oder den Bildinhalt betreffender Eigenschaften auf mehr oder weniger spezifische historische Epochen. ${ }^{153}$ Hiermit ist gemeint, dass entweder die Schärfe, der Bildausschnitt, die Verteilung der Kontraste, das Filmkorn oder die Farbigkeit der Bilder, aber auch das Format (ohne dass man weiß, ob sie bearbeitet oder beschnitten wurden) auf eine bestimmte Episode der Fototechnik hinweisen oder dass die dargestellten Personen, ihre Kleidung, ihr Haarschnitt und ihre Präsentation für die Kamera, die erkennbare Architektur, die Objekte und vor allem auch Fahrzeuge Hinweise darauf geben, wann die Bilder entstanden sein könnten. Bei fast allen Fotos handelt es sich offensichtlich um Bilder aus dem privaten Bereich, wie sie in Familienalben zu finden sind. Auf den meisten Bildern sind Menschen zu sehen, die entweder für die Kamera posieren, in Alltagssituationen oder bei besonderen Gelegenheiten wie Festen, Picknicks

Regimentstochter (2005), welche aus 36 Opern- und Theaterprogrammen der NS-Zeit besteht, aus denen die Hakenkreuzsymbole herausgeschnitten wurden, oder zuletzt die auf Flohmärkten gesammelten Postkarten, die Eingang in das Filmprojekt FILM (2012) fanden.

153 Der Ausdruck >historischer Index` wird hier nicht in Analogie zur fotografischen Indexikalität verwendet, sondern bezeichnet zunächst nur die Tatsache, dass auf die Vergangenheit verwiesen wird. Walter Benjamin verwendete den Begriff, um dialektische Bilder von der Phänomenologie einerseits, von >geisteswissenschaftlichen K Kategorien wie dem Habitus oder dem Stil andererseits abzugrenzen: »Der historische Index der Bilder sagt nämlich nicht nur, daß sie einer bestimmten Zeit angehören, er sagt vor allem daß sie erst in einer bestimmten Zeit zur Lesbarkeit kommen « - und zwar im »Jetzt einer bestimmten Erkennbarkeit« (Benjamin, Das Passagen-Werk, Bd. 1, S. 577 f.). 
oder Ähnlichem festgehalten sind oder der Kamera Objekte präsentieren (Abb. 15-19). Die professionelle Qualität der Aufnahmen variiert stark, was wiederum ein Hinweis auf den privaten Ursprung der Fotos sein kann. Fast alle Bilder haben Kratzer und Flecken, zeigen Flusen und störende Partikel, die während der Entwicklung auf das Papier gelangten, sie haben Belichtungsfehler, Fehler in der Schärfe, sind schief oder, bezogen auf das Bildobjekt, falsch ausgeschnitten. ${ }^{154}$ »Das Material drängt sich auf $«,{ }^{155}$ wie Peter Geimer das Sichtbarwerden des fotografischen Prozesses durch das zufällige Auftreten von Bildfehlern oder Störungen genannt hat, beispielsweise bei der sorgsam komponierten Gegenlichtaufnahme eines Mannes beim Geigenspiel (Abb. 2o). Manche Bilder tragen sichtbare Verweise auf die chemischen und physikalischen Entstehungsbedingungen, manche auf ihre Vorbesitzer: Mark Godfrey hebt die Schwarz-Weiß-Aufnahme einer Gruppe Frauen und Männer in Uniform heraus, die lächelnd und sorgfältig in drei Reihen aufgestellt für den Fotografen posieren. Es wäre eine unspektakuläre, stereotype Fotografie, wie sie in Jahrbüchern zu finden ist, wären nicht die Gesichter zweier Frauen mit blauem Stift übermalt und dadurch unkenntlich gemacht worden. Ein anderes Bild trägt auf dem Gesicht eines kleinen Jungen einen deutlichen Fingerabdruck. Beides sind Spuren eines früheren Gebrauchs, die die körperliche Kontaktaufnahme des Vorbesitzers mit dem Bild belegen und der Indexikalität der Fotografie auf die Situation der Aufnahme einen weiteren Index hinzufügen, den Verweis auf die emotionale, affektive Bindung einer Person an die Bilder als historische Objekte. ${ }^{156}$

Alle Bilder in $F L O H$ weisen solche Eigentümlichkeiten auf, die den Blick des Betrachters / der Betrachterin fesseln und Hinweise darauf geben, was Tacita Dean selbst an dem Bild interessiert haben könnte; es sind Fotografien, die ein punctum im Sinne Barthes' haben - ein Detail, eine besondere

154 Margaret Iversen hat die zahlreichen sogenannten Fehler, die sich auf den Fotografien finden, als ein mögliches Auswahlkriterium Deans gedeutet: »The photos are a regular inventory of technical errors: [...] It is as though the condition of the medium were being explored by illustrating everything that can go wrong.« (Margaret Iversen, »Analogue. On Zoe Leonard and Tacita Dean«, in: Critical Inquiry 38/4, Sommer 2012, S. 796-818, S. 813). Diese Interpretation degradiert jedoch das Kompendium FLOH zur Illustration einer (von Dean sicher nicht angestrebten) teleologischen Geschichte der Fotografie, die auf eine Perfektionierung des Abbildungsverhältnisses ausgerichtet ist, und reduziert außerdem die Auswahl der Künstlerin auf ein schlichtes Sammeln von Fehlern. Sie wird der Arbeit und den einzelnen Bildern nicht gerecht.

155 Peter Geimer, Bilder aus Versehen. Eine Geschichte fotografischer Erscheinungen, Hamburg 2010, S. 85 .

156 Vgl. Mark Godfrey, »Photography Found and Lost. On Tacita Dean's Floh«, in: October 114, Herbst 2005, S. 90-119, S. 110-112. 

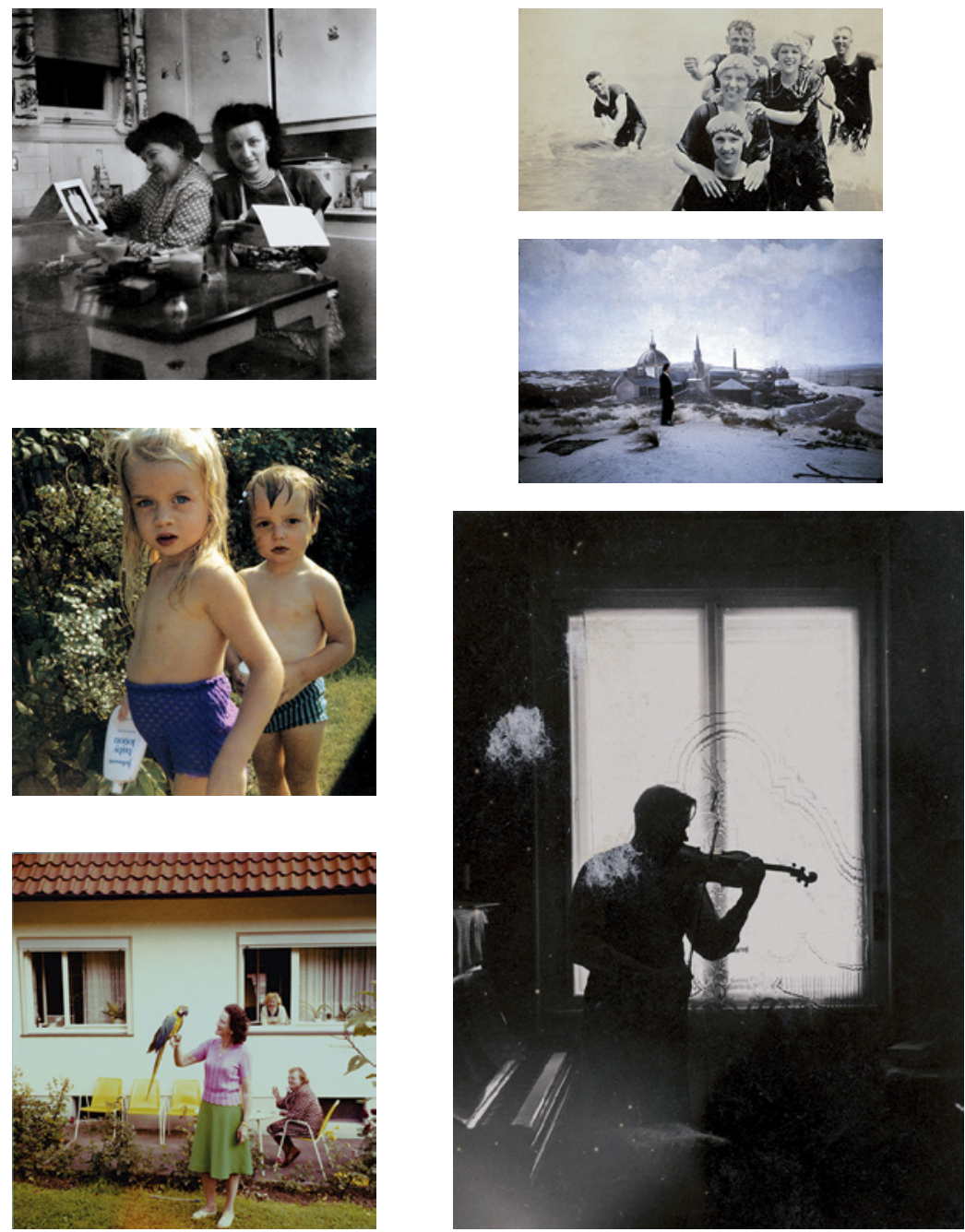

Abb. 15-2O Tacita Dean, Fotografien aus dem Künstlerbuch FLOH, 2001 
Kuriosität oder eine bildliche Auffälligkeit -, das eine spezielle Verbindung zur Vergangenheit des Fotos knüpft. ${ }^{157}$ Wie kann diese semiotische Bedeutungsebene mit der bildlichen Deixis der Fotografien zusammengehen? Zwei Aspekte sollen im Folgenden für die hier im Mittelpunkt stehende Frage nach der Zeitlichkeit bei der Arbeit mit gefundenen Materialien betrachtet werden: einerseits die zeitliche Struktur der Sammlung, ihre Ordnung, Typologie und die Anordnung im Buch, aber auch die von Dean unterstrichene Unabgeschlossenheit des Projekts; andererseits die hier schon angedeutete Zeitlichkeit der Bilder selbst, deren Historizität als Eigenschaft der Fotografie an sich, aber darüber hinaus als bildliches Faktum zutage tritt.

\title{
Lost, Found, Rearranged. Die Sammlung
}

\author{
»Ich möchte, dass sie das Schweigen des \\ Flohmarktes bewahren; das Schweigen, das sie \\ umgab, als ich sie gefunden habe; das Schweigen \\ des verlorenen Objekts. $« 158$
}

Tacita Deans Sammlung FLOH könnte heterogener nicht sein: Neben sorgfältig oval ausgeschnittenen, sepiafarbenen Porträts aus der Frühzeit der Fotografie finden sich verwackelte Schnappschüsse, deren mangelnde Schärfe, schlechte Blitzausleuchtung und kontrastreiche Farbgebung auf eine Kleinbildkamera der 196oer oder 1970er Jahre hinweisen. Neben der peniblen Dokumentation, wie ein übergewichtiger Mann in Anzug und Hornbrille sich hinter das Steuer eines viel zu kleinen gelben Sportwagens zwängt (Abb. 21), ${ }^{159}$ steht die Aufnahme eines Schmetterlings im Schnee oder eines Manns, der verloren in einer Art Filmkulisse steht (Abb. 19). In seinem umfangreichen Essay über die Arbeit hat Mark Godfrey eine (vergebliche) Systematisierung der Themen und Typen anhand der Anordnung der Fotografien versucht und dabei die diskontinuierliche Zeiterfahrung beim Blättern im Buch beschrieben: Jede vermeintliche Kontinuität über formale oder inhaltlich zu knüpfende Verbindungen einander gegenübergestellter oder in Reihe befindlicher Fotos

\footnotetext{
$157 \mathrm{Zu}$ den verschiedenen Bedeutungsebenen des punctums bei Barthes siehe einmal mehr die Ausführungen in Arndtz, Philosophie der Fotografie.

158 Tacita Dean, »FLOH «, in: Tacita Dean. seven books grey, Bd. 4: Ausgewählte Schriften 19922011, hg. v. Museum moderner Kunst Stiftung Ludwig Wien, Steidl Publishers, Göttingen 2011, S. 5 .

159 Diese Serie trägt einen von der Künstlerin ausgewählten Titel, da sie Teil einer Sammlung von 25 Fotografien ist, die von Dean als Edition von 10 Exemplaren vergrößert und als Digitalprint gesondert verlegt wurden.
} 

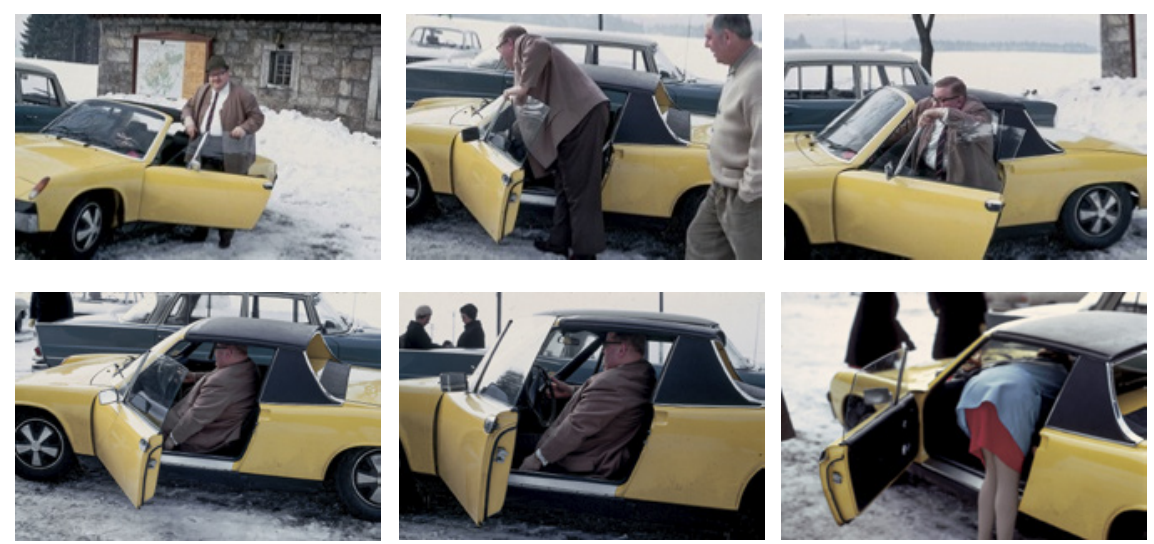

Abb. 21 Tacita Dean, Yellow Car (Diptych), Fotografien aus dem Künstlerbuch FLOH, 2001

wird durch Brüche, Sprünge und einen durchbrochenen Rhythmus jäh wieder aufgehoben. ${ }^{160}$ Es ist, als würde man selbst durch die Kisten alter Fotografien an einem Flohmarktstand blättern, wobei manche den Blick stärker fesseln als andere. Im Gegensatz zu ihren Filmen, die in der Regel durch die langen Einstellungen einen langsamen Rhythmus haben, lädt $F L O H$ dazu ein, vorund zurückzuspringen, Bilder zu vergleichen und Zusammenhänge herzustellen. ${ }^{161}$ Versucht man, die Ordnung, die Dean ihren gefundenen historischen Bildern gab, zu benennen, kommen Kategorien wie formale, bildliche und inhaltliche Ähnlichkeit, visuelle Kontraste, narrative Kontinuität oder Brüche in den Sinn. Man fühlt sich an Bildersammlungen und Arrangements der Vor- und Nachkriegsavantgarde erinnert, die jedoch selbst nach ganz unterschiedlichen Prämissen arbeiteten. In seinem Essay »Warburgs Vorbild?«162 konstatiert Benjamin H. Buchloh einen Bruch zwischen den scheinbar sich ähnelnden Sammlungs- und Ordnungstätigkeiten von Aby Warburg in seinem Mnemosyne-Atlas um 1925, der Collageästhetik der Surrealisten und den Fotomontagen der Dadaisten sowie den archivalisch arbeitenden Fotokünstlern

16o Godfrey, »Photography Found and Lost«, S. 92 f.

161 Ebd., S. 96: »As we turn each page with anticipation, we relive Dean's experience of coming across the images in the flea market, each moment of enchanted discovery. But the temporal experience of flipping through the book is far from fluid. Dean's films are calmly paced, each still shot holding its image for what always feels like a generous amount of time before the cut to the next one. With Floh, however, the rhythm is rather jolty-it is more a matter of delays and quick restarts.«

162 Benjamin H. D. Buchloh, »Warburgs Vorbild? Das Ende der Collage/Fotomontage im Nachkriegseuropa «, in: Ingrid Schaffner, Matthias Winzen (Hg.), Deep Storage. Arsenale der Erinnerung. Sammeln, Speichern, Archivieren in der Kunst, Ausstellungskatalog Haus der Kunst München u. a., 1997, München 1997, S. 50-6o. 
der 196oer und 1970er Jahre wie Bernd und Hilla Becher, Gerhard Richter oder Marcel Broodthaers. Seine Typologie der Sammlungsorganisation umfasst die Logik der Collage, die auf Schock und Verfremdung beruht und die 2oer Jahre kennzeichnete, den kulturwissenschaftlichen Ansatz einer »archivalische[n] Montage « bei Warburg, die »unbegrenzte Heterogenität« von Richters Atlas, die in ein streng serielles Ordnungssystem gepresst wird, dem gegenüber die didaktische, »absurd limitierte Homogenität« von Broodthaers' Section Publicité und die melancholische, zwanghafte Wiederholung der Variation des Immergleichen bei Bernd und Hilla Becher. ${ }^{163}$ Mit keinem dieser Ansätze kann Deans $F L O H$ konkret verglichen werden, basiert die Auswahl ihrer Materialien doch auf einem ungenannten aleatorischen Prinzip der Entdeckung und Kombination ausschließlich nach visuellen Kriterien: »There seems little logic to this design, no archival or serial principles that might be obeyed. And Dean is aware of this: working with Ridgewell on the design, Floh, she says, was laid out intuitively: >we just did it totally visually, refusing all categories.« «164

Für die Auswahl und Anordnung der Fotografien scheinen also vor allem die Prinzipien der Zufälligkeit und der visuellen Intuition leitend gewesen zu sein. Dabei ist es wichtig, dass gerade die Auswahl der Bilder mehr verbirgt, als sie verrät: Es ist unklar, welche Fotografien zusammen gefunden wurden oder, bis auf wenige abgedruckte Ausnahmen, ob sie Serien entstammen. Auch wissen wir nicht, ob Dean jeweils aus mehreren ähnlichen Abzügen einen auswählte oder die Bilder an sich >Unikate< darstellen. Für das Fotobuch ist dies insofern von Belang, als alle Bilder durch ihre Wiederverwertung eine neue Bedeutung, nämlich als Teil dieser Sammlung, zugesprochen bekommen. Als Teil einer individuellen Auswahl kann jedes einzelne Bild stets wieder dem Prozess der Neubewertung entzogen werden, einen anderen Platz einnehmen, in Korrespondenz mit neuen Bildern stehen. Dass der Prozess des Sammelns, sobald sich das Korpus einer Sammlung bildete, eine zeitliche und räumliche Begrenzung haben würde, beunruhigte die Künstlerin:

Erst nach einiger Zeit wurde mir klar, dass ich gerade dabei war eine Sammlung anzulegen, und nichts beunruhigt den Sammler mehr als die Aussicht auf einen >Abschluss<; die Erkenntnis, dass es eine >Endfassung< gibt - und damit ein mögliches Ende des Sammelns. [...] Mittlerweile bin ich aber zu dem Schluss gekommen, dass es keine `Endfassung « dieser Sammlung gibt und auch niemals geben kann; dass $\mathrm{FLOH}$ in diesem Kontinuum existiert und hoffentlich eines Tages, herrenlos und stillschweigend, zu seinen Ursprüngen am Flohmarkt zurückkehren wird. ${ }^{165}$

163 Vgl. ebd., S. 55 f.

164 Godfrey, »Photography Found and Lost «, S. 96.

165 Dean, »FLOH«. 
Deans Wunsch nach einer Unabgeschlossenheit des Projekts steht die eigene Entscheidung gegenüber, die Sammlung oder Teile davon 2001 als Buch zu veröffentlichen. Das Zitat unterstreicht den Glauben daran, dass das einzelne Bild seine eigene Zeitlichkeit bewahren kann, als Objekt ungebunden zeitlich existiert. Es unterstreicht auch, dass Dean, ganz im Sinne von Foucaults Unterscheidung in der Archäologie des Wissens, die Dokumente anderer Zeiten als Monumente wahrnimmt, als von Sinnstiftung und Deutung unabhängige zeitliche Entitäten. ${ }^{166}$ Sie verwandelt die Fragmente der Vergangenheit nicht wie die klassische Geschichtsschreibung in sprechende Dokumente, die ausschließlich der positivistischen Spurensuche dienen, sondern isoliert sie mit archäologischer Präzision. Sie macht dadurch auch ihren geschichtlichen Zugang zum gefundenen Material deutlich, nämlich ein Sammeln, Auswählen und Ausstellen gerade nicht im Sinne einer klassischen Historiografie, welche die Geschichte als Ergebnis der Rekonstruktion und Kontinuität von Ereignissen beschreibt, in deren narrativem Verlauf Objekte als Zeugen, Belege oder Verweise eingebunden sind, sondern mit dem Ziel einer Öffnung des Materials hin auf eine andere, fiktive, aber ebenso mögliche Geschichte über die Isolierung und Dekontextualisierung der Objekte.

\section{Vergangenheit als bildliches Faktum der Fotografie}

Deans Arbeit weist uns auf die bildtheoretische Frage der Unterscheidung zweier geschichtlicher Dimensionen des Bildes hin, denn sie konfrontiert die Historizität der Fotografie als Medium der Spur mit einer davon zu unterscheidenden bildlichen Historizität. Diese Doppelheit betrifft jedoch nicht nur die Historizität, sondern hat ihren Ursprung in der grundlegenden Doppelheit der bildlichen Darstellung, welche immer zugleich etwas darstellt und sich darstellt, wie Martina Dobbe zusammenfasst: »Bildliches Sehen meint stets das Sehen des Dargestellten wie das Sehen des Mediums, bildliches Zeigen meint das Zeigen von etwas wie gleichermaßen und zugleich das Sich-Zeigen der Darstellung. ${ }^{167}$ Geht man von einem wahrnehmungstheoretischen Ansatz aus, dass also Bilder nicht in erster Linie gelesen werden, sondern eine eigene Dimension der Sichtbarkeit erzeugen, muss nach Husserl und Fiedler sogar dreigeteilt unterschieden werden zwischen dem Darstellenden, der Darstellung selbst (als Bildobjekt) und dem Dargestellten (als Bildsujet). ${ }^{168}$

166 Foucault, Archäologie des Wissens, S. 13-15.

167 Martina Dobbe, »Zeigen als faire voir. Für eine Bildtheorie des Fotografischen«, in: Gottfried Boehm, Sebastian Egenhofer, Christian Spies (Hg.), Zeigen. Die Rhetorik des Sichtbaren, München 2010, S. 158-178, S. 158.

168 Vgl. Lambert Wiesing, »Die Hauptströmungen der gegenwärtigen Philosophien des Bildes«, in: ders., Artifizielle Präsenz. Studien zur Philosophie des Bildes, Frankfurt am Main 2005 , S. $17-36$, S. 33 . 
Für die Fotografie als abbildendes Medium ist das Verhältnis besonders prekär, denn es trägt die unauslöschbaren Hinweise auf das, was gewesen ist (»ça-a-été«), beziehungsweise die Tatsache, dass das auf dem Bild Sichtbare gewesen ist, in sich. Die Bezugnahme auf den festzustellenden, aber nicht unbedingt genau festzulegenden Zeitpunkt der Aufnahme - die Transparenz des Mediums Fotografie, das seine eigene Medialität zeigt - kollidiert mit ihrer Bildlichkeit, die sich in der Tatsache manifestiert, dass das Foto selbst als Bild in Erscheinung tritt, eine Sichtbarkeit hat, die aber eine/n gegenwärtige/n Betrachter/in erfordert. Der von Wiesing beschriebene Konflikt zwischen der semiotischen Bestimmung der Darstellung als Bildinhalt und der wahrnehmungstheoretischen Bestimmung als Bildobjekt manifestiert sich in historischen Fotografien besonders anschaulich, da Fotografien qua ihrer materialen Beschaffenheit eine zeitlich verweisende Dimension haben. Wichtig ist hier jedoch die Tatsache, dass die Fotografien sich nicht darin erschöpfen, also nicht nur als Indizes zu lesen sind, sondern in ihrer zur Schau getragenen Geschichtlichkeit den Betrachter / die Betrachterin von sich aus auf seine/ihre gegenwärtige Position des Schauenden hinweisen. Das Sehen des Bildes erzeugt ein »sinnliche[s] Gegenwartsbewußtsein «, 169 und die Wahrnehmung des Bildobjekts macht den Gegenstand der Darstellung präsent, aber als imaginären Gegenstand: »Denn der Betrachter eines Bildes sieht, daß er ein Bild von etwas und nicht die Sache selbst sieht.«170 Die Auswahl der Fotografien für $\mathrm{FLOH}$ ist insofern ein aussagekräftiges Beispiel für die historische Ambivalenz der gefundenen Fotografie, als sie tatsächlich aufgrund der zahlreichen >Bildfehler< und der puncta den Betrachter/innen bewusst machen, dass sie als gegenwärtige Rezipient/innen des Bildes dieses >als Bild < der Vergangenheit sehen. Es ist weniger transparent für die Erzählung der Geschichte als opak - die Fotografie zeigt sich als ein Medium, das sich selbst durch seine Fehler sichtbar macht. Die Betrachter/innen erkennen die Bilder als historisch und definieren damit zugleich unsere Position in der Jetztzeit, in einem unbekannten Abstand zum Zeitpunkt des fotografischen Aktes. Die schweigenden, »verlorenen Objekte « bezeugen in der vorliegenden Kombination als Buch einzig, dass sie von Dean gefunden, sortiert, kombiniert und veröffentlich wurden. Ihr historischer Status als Zeugen der Vergangenheit ist jedoch ambivalent, da sie die Tatsache ihrer Entstehung belegen, der Kontext ihrer Entstehung jedoch verloren ist - die Spur verweist ins Leere. Sie belegen jedoch, und hier treffen sich die Zeitlichkeit und die Geschichtlichkeit des gefundenen Bildes, dass das jeweilige Ereignis des Gesehenwerdens

169 Ebd., S. 34.

170 Ebd. 
diese historische Unbestimmbarkeit im gegenwärtigen Akt der Wahrnehmung bewusst werden lässt und die Vergangenheit so als bildliches Faktum in Erscheinung tritt.

\section{Fazit}

Die Arbeit mit gefundenem Material ist, wie nach der Betrachtung dieser sehr unterschiedlichen Beispiele deutlich wird, keine Methode, sich die Vergangenheit des Bildes in der Gegenwart anzueignen und sich den historischen Index des Materials zunutze zu machen - das Wort Methode oder Verfahren wäre überdies zu einengend für die Vielzahl an Erscheinungsformen, von denen hier nur Fotografie und bewegtes Bild herausgegriffen wurden. ${ }^{171}$ Vielmehr ist die Arbeit mit Found Footage Ausweis einer Haltung: gegenüber der eigenen Rolle als künstlerischem Subjekt, gegenüber dem Konzept eines Werks als historisch abgeschlossen, gegenüber der Geschichte des Films und der Fotografie. Künstler wie Gordon, Coleman, Müller/Giradet und Arnold behandeln Film dabei als kulturelles Artefakt, das in der ästhetischen Reflexion zu neuem Leben erwacht. Nicht die Speicherung und Repräsentation von realen Ereignissen steht dabei im Vordergrund (eine Lesart als Quelle wird vermieden), sondern die Aktivierung von Erinnerungsprozessen im durch die Betrachter/ innen erfahrenen und in der ästhetischen Erfahrung stets aktualisierten Beziehungsgefüge unterschiedlicher Zeitschichten. Gerade weil sie die zeitliche und die räumliche Dimension des filmischen Dispositivs erweitert, kann die Arbeit mit Found Footage im Kunstraum dazu beitragen, nicht nur die filmischen Bilder, sondern auch ihre vielfältigen medialen Erscheinungsformen kritisch zu reflektieren.

Die mit der Wiederverwendung von gefundenen Fotografien im Fotobuch FLOH von Tacita Dean vergleichende Betrachtung hat gezeigt, dass hier dieselbe besondere Verweisstruktur des fotografischen Bildes wie im FoundFootage-Film zum Tragen kommt. Es treffen sich zwei Arten der Historizität, die indexikalische und die bildliche, die den Betrachter oder die Betrachterin im Zuge der Wahrnehmung der Spuren der Vergangenheit im Bild auf seine oder ihre Jetztzeit verweisen. Ebenso wie der Film ist das Buch linear angelegt,

\footnotetext{
171 Nur der Vollständigkeit halber soll hier darauf verwiesen werden, dass die Einbindung gefundener Materialien in künstlerische Werke der Moderne natürlich keine alleinige Domäne des Films oder der Fotografie darstellt. Insbesondere in den Collagen und Installationen der Dada-Künstler/innen und der Pop Art, zum Beispiel Hannah Höch und Robert Rauschenberg, war dies aus unterschiedlichen politischen und ästhetischen Motivationen heraus verbreitet; zu erwähnen sind auch, neben vielen anderen, Joseph Cornell, Dieter Roth, John Bock, Annette Messager oder Christian Boltanski, die in Skulpturen, Objekten und Installationen mit vorgefundenen Materialien arbeiten.
} 
es erlaubt jedoch durch das Blättern eine aktive Verknüpfung der Bilder. Der vergleichenden Anschauung und dem Versuch beim Betrachten des Fotobuchs, sich historisch anhand bildlicher Merkmale zu orientieren, entspricht im Film die verbindende Rolle der Erinnerung, frühere mit späteren Bildern und Einstellung zu verknüpfen. Bei den Spielfilmadaptionen von Müller/Girardet und Gordon wird zudem die Erinnerung an bekannte Filme miteinbezogen. Die Arbeit am bildlichen Fragment, die Neusortierung und Umdeutung der Bilder stellt abschließend die Frage, ob die hier besprochenen Werke als Teil einer künstlerischen alternativen Geschichtsschreibung gelten können. 